\title{
MRI of atherosclerosis : identification and quantification of carotid plaque components
}

Citation for published version (APA):

Cappendijk, V. C. (2007). MRI of atherosclerosis : identification and quantification of carotid plaque components. [Doctoral Thesis, Maastricht University]. Universitaire Pers Maastricht. https://doi.org/10.26481/dis.20070323vc

Document status and date:

Published: 01/01/2007

DOI:

10.26481/dis.20070323vc

Document Version:

Publisher's PDF, also known as Version of record

\section{Please check the document version of this publication:}

- A submitted manuscript is the version of the article upon submission and before peer-review. There can be important differences between the submitted version and the official published version of record.

People interested in the research are advised to contact the author for the final version of the publication, or visit the DOI to the publisher's website.

- The final author version and the galley proof are versions of the publication after peer review.

- The final published version features the final layout of the paper including the volume, issue and page numbers.

Link to publication

\footnotetext{
General rights rights.

- You may freely distribute the URL identifying the publication in the public portal. please follow below link for the End User Agreement:

www.umlib.nl/taverne-license

Take down policy

If you believe that this document breaches copyright please contact us at:

repository@maastrichtuniversity.nl

providing details and we will investigate your claim.
}

Copyright and moral rights for the publications made accessible in the public portal are retained by the authors and/or other copyright owners and it is a condition of accessing publications that users recognise and abide by the legal requirements associated with these

- Users may download and print one copy of any publication from the public portal for the purpose of private study or research.

- You may not further distribute the material or use it for any profit-making activity or commercial gain

If the publication is distributed under the terms of Article $25 \mathrm{fa}$ of the Dutch Copyright Act, indicated by the "Taverne" license above, 
MRI of atherosclerosis -

Identification and quantification of carotid plaque components 
ISBN 978-90-5278-605-6

Copyright (c) VC Cappendijk, Maastricht 2007

Printed by: Datawyse

Font: Univers-55 10 pnt

Line interval: exact 15 pnt

Cover picture: Jardín Botánico de Cienfuegos, Cienfuegos, Cuba

Back picture: Carotid plaque of symptomatic patient (top), Carotid plaque of asymptomatic patient (bottom)

The publication of this thesis was financially supported by:

EV3

Guerbet Nederland B.V.

Kodak Health Group

Medis medical imaging systems bv

Medtronic Bakken Research Center B.V.

Pfizer bv

Philips Medical Systems

Sanofi-aventis en Bristol-Meyers Squibb

Tyco Healthcare Nederland B.V. 


\section{$\mathrm{MRI}$ of atherosclerosis}

Identification and quantification of carotid plaque components

\section{Proefschrift}

ter verkrijging van de graad van doctor

aan de Universiteit Maastricht, op gezag van de Rector Magnificus,

Prof. mr. G.P.M.F. Mols,

volgens het besluit van het College van Decanen,

in het openbaar te verdedigen

op vrijdag 23 maart 2007 om 14.00 uur

door

Vincent Cappendijk

Geboren op 14 augustus 1970 te Eindhoven

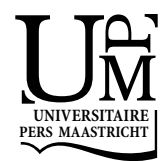


Promotores:

Prof. dr. J.M.A. van Engelshoven

Prof. dr. M.J.A.P. Daemen

Copromotores:

Dr. M.E. Kooi

Dr. S. Heeneman

Beoordelingscommissie:

Prof. dr. M. Limburg (voorzitter)

Prof. dr. A.G. Bruggeman

Dr. L. Hofstra

Prof. dr. P.J.E.H.M. Kitslaar

Dr. A. van der Lugt (Erasmus Medisch Centrum Rotterdam) 


\section{Contents}

Chapter 1 General introduction ........................................................ 7

Chapter 2 In vivo detection of hemorrhage in human atherosclerotic plaques with Magnetic Resonance Imaging

Published in JMRI 2004;20:105-110.

Chapter 3 Assessment of human atherosclerotic carotid plaque components by multisequence MR imaging: Initial experience

Published in Radiology 2005;234:487-492

Chapter 4 In vivo MR quantification of the lipid-rich necrotic core in carotid atherosclerotic plaque Submitted to European Radiology. 49

Chapter 5 Magnetic resonance imaging quantification of lipidrich necrotic core size in carotid atherosclerotic plaque of symptomatic and asymptomatic patients Submitted to European Radiology

Chapter 6 Accumulation of ultrasmall superparamagnetic particles of iron oxide in human atherosclerotic plaques can be detected by in vivo magnetic resonance imaging

Published in Circulation 2003; 107:2453-2458

Chapter $7 \quad$ General discussion........................................................... 107

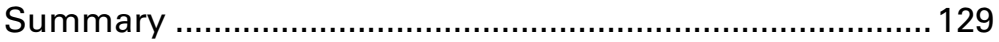

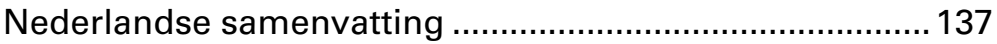

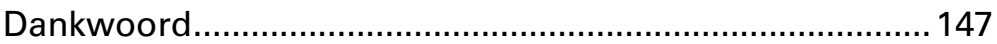

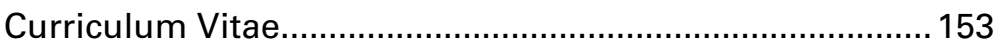

List of publications ........................................................... 157 



\section{Chapter}

1

\section{General introduction}

$\mathrm{MRI}$ of atherosclerosis -

Identification and quantification of carotid plaque components 
Stroke is one of the leading causes of mortality and morbidity in the Western society. Two large clinical trials, the North American Symptomatic Carotid Surgery Trial (NASCET) and the European Carotid Surgery Trial (ECST), ${ }^{1,2,3,4,5}$ showed that patients with symptomatic atherosclerotic carotid disease and a stenosis larger than $70 \%$ benefit from endarterectomy. However, $80 \%$ of these patients will not suffer stroke with medical treatment alone and are exposed to substantial mortality and morbidity due to endarterectomy with a risk of $7.1 \%$ of stroke and/or death. ${ }^{6}$ For asymptomatic patients with high-grade carotid stenosis the benefit of endarterectomy was only marginal. ${ }^{7}$ Therefore, it would be very important to identify additional risk factors of stroke to come to a better subgroup selection of patients at risk.

Today, the indication for endarterectomy and carotid stenting for atherosclerotic carotid disease focusses on the degree of stenosis and symptomatology. The underlying pathology of patients with carotid stenosis is the presence of an atherosclerotic plaque. The earliest atherosclerotic lesion is the fatty streak, which consists of lipid-laden monocytes and macrophages together with T lymphocytes. A fatty streak may progress to intermediate and advanced lesions which contain extracellular lipid and an evolving necrotic core covered by a fibrous cap that separates the blood from the thrombogenic plaque contents. ${ }^{8}$ Infiltration of inflammatory cells in carotid plaque may be a critical step in promoting rupture of the fibrous cap, followed by embolization or carotid occlusion. ${ }^{9}$ It is well known from pathological studies that the composition of the vessel wall, i.e. the atherosclerotic plaque, is important to identify plaques, which potentially cause thrombo-embolic events. ${ }^{10,11,12,13}$ Generally, a large lipid-rich necrotic core (LRNC), intra-plaque hemorrhage and plaque inflammation are described as features of a vulnerable, ruptureprone plaque. Unfortunately, there are no established diagnostic tools to visualize plaque components and plaque inflammation in vivo with sufficient accuracy. Positron emission tomography (PET) and single photon emission computed tomography (SPECT) have a very limited spatial resolution and a particular radiotracer is only able to identify one single aspect of complex atherosclerotic lesions. ${ }^{14,15,16}$ In vivo ultrasound plaque characterization lacks consistency and accuracy ${ }^{17,18}$ and is therefore not very promising for this purpose. Many catheter-based techniques have 
been introduced (intravascular $\mathrm{MRI}^{19,20,21}$, optical coherence tomography ${ }^{20,22}$, palpography ${ }^{23}$, thermography ${ }^{20,24}$ ), but are invasive and therefore not suitable for screening purposes or large scale follow up studies. An exception is intravascular ultrasound ${ }^{20,25,26,27,28}$ (IVUS), which is generally performed as a small additional procedure during an invasive coronary X-ray angiograpy. IVUS showed to be a reliable modality for large scale follow up in coronary arteries. Schoenhagen et al ${ }^{28}$ demonstrated coronary arterial wall changes in serial IVUS observations during lipid-lowering therapy. However, these invasive catheter based techniques were not within the scope of our studies and will therefore not be discussed further in this thesis.

At the start of the present $\mathrm{PhD}$ study several research groups ${ }^{29,30}$ had shown great opportunities for MRI to study human atherosclerotic carotid vessel wall disease in vivo. MRI proofed to have high potential for atherosclerotic plaque imaging, because it combines many aspects warranted for atherosclerotic plaque imaging, such as high soft tissue contrast and high in-plane resolution and high reproducibility and repeatibility. Previous studies ${ }^{29,30}$ showed the feasibility of MRI to differentiate various carotid plaque components using a combination of several MR pulse sequences, but had not determined the size of these components. These studies were able to identify the lipid-rich necrotic core, but had difficulties to identify intra-plaque hemorrhage. Because a large size of the LRNC is associated with vulnerable plaque, quantification is important. Although those initial in vivo MRI studies ${ }^{29,30}$ qualitatively determined the relative signal intensities (rSls) for the different plaque components as hypo-, iso- and hyperintensive in comparison with surrounding muscle tissue in various MR pulse sequences, the optimal MR pulse sequence combination and corresponding cutoff rSls for identification of a certain plaque component was not investigated. Moreover, published rSI cutoff points were qualitative observations, while quantitative rSls had not been measured.

An increased number of macrophages in an atherosclerotic plaque is related to plaque vulnerability. Initial post-USPIO (ultrasmall superparamagnetic particles of iron oxide) MR imaging showed to have potential to visualize macrophage-rich regions as signal voids in the rabbit 
aortic wall, ${ }^{31,32}$ but post-USPIO MR imaging of atherosclerotic lesions was never performed in humans at start of our studies.

Future implementation of MR imaging of atherosclerotic plaques in clinical practice will require an objective diagnostic test, and therefore besides identification of the vulnerable plaque components, quantification and standardized image assessment will be very important. Moreover, quantification might provide surrogate endpoints for future (multicenter) clinical (drug) trials, which might reduce the enormous number of patients needed in trials with clinical endpoints.

The hypothesis of this thesis was that vulnerable atherosclerotic plaque characteristics can be identified and quantified with standardized in vivo MRI. To be able to perform this study over one-hundred patients with carotid stenosis larger than $70 \%$ were recruited. All patients gave written informed consent.

\section{Outline of this thesis:}

In chapter 2, we investigated whether in vivo MRI can accurately detect the high-risk component intra-plaque hemorrhage using a novel pulse sequence for carotid plaque imaging. The performance of the novel MR pulse sequence, a T1 weighted ( $w$ ) turbo field echo (TFE) sequence for the identification of intra-plaque hemorrhage, was compared with that of a more common used T1w turbo spin echo MR pulse sequence.

In chapter 3, we determined the optimal MR pulse sequence combination for the identification of atherosclerotic plaque components (calcification, lipid core, intra-plaque hemorrhage, fibrous tissue, lipid-rich necrotic core) with standardized MR image assessment of patients with high-grade carotid stenosis. A strict algorithm was developed based on five MR pulse sequences. Logistic regression analysis determined which MR pulse sequence combinations and relative signal intensity cut-off values (qualitative and semi-quantitative) were optimal for the prediction of the main plaque components (calcification, lipid core, intra-plaque hemorrhage, fibrous tissue, lipid-rich necrotic core).

In chapter 4, we investigated whether in vivo MRI was able to accurately quantify these (vulnerable) atherosclerotic carotid plaque 
components. Therefore, quantification of plaque components according to the strict algorithm and quantification of the lipid-rich necrotic core based solely on T1w TFE images were tested prospectively on a larger series of symptomatic carotid patients.

In chapter 5, we evaluated whether our standardized MR image assessment for carotid plaque had potential to measure the LRNC size as risk factor of stroke. For this purpose it was tested whether the amount of lipid-rich necrotic core in carotid plaques quantified with MRI differs in symptomatic and asymptomatic patients. Three individual MR readers, blinded for any other medical information, assessed the plaques of all patients using T1w TFE images.

In chapter 6, we investigated in a proof of concept study whether macrophages in human carotid plaques showed uptake of USPIO contrast agent and whether in vivo MRI can visualize plaque regions with macrophages. Therefore, carotid endarterectomy specimens were studied microscopically and electron-microscopically to assess the presence of USPIOs in macrophages. Plaques were categorized as rupture prone/ruptured versus stable and it was investigated whether uptake of USPIO occurred preferentially in one of these categories. It was studied whether uptake of USPIO lead to signal decreases in the MR images

In chapter 7, the current status of MR plaque imaging and future directions of atherosclerotic plaque imaging will be discussed.

Chapter 8 , presents a summary of this thesis. 


\section{References}

1. Beneficial effect of carotid endarterectomy in symptomatic patients with high-grade carotid stenosis. North American Symptomatic Carotid Endarterectomy Trial Collaborators. N Engl J Med. 1991;325:445-453.

2. MRC European Carotid Surgery Trial: interim results for symptomatic patients with severe (70-99\%) or with mild (0-29\%) carotid stenosis. European Carotid Surgery Trialists' Collaborative Group. Lancet. 1991;337:1235-1243.

3. Randomised trial of endarterectomy for recently symptomatic carotid stenosis: final results of the MRC European Carotid Surgery Trial (ECST). Lancet. 1998;351:1379-1387.

4. Barnett HJ, Taylor DW, Eliasziw M, Fox AJ, Ferguson GG, Haynes RB, Rankin RN, Clagett GP, Hachinski VC, Sackett DL, Thorpe KE, Meldrum $H E$, Spence JD. Benefit of carotid endarterectomy in patients with symptomatic moderate or severe stenosis. North American Symptomatic Carotid Endarterectomy Trial Collaborators. N Engl J Med. 1998;339:1415-1425.

5. Rothwell PM, Eliasziw M, Gutnikov SA, Fox AJ, Taylor DW, Mayberg MR, Warlow CP, Barnett HJ. Analysis of pooled data from the randomised controlled trials of endarterectomy for symptomatic carotid stenosis. Lancet. 2003;361:107-116.

6. Rothwell PM, Slattery J, Warlow CP. A systematic review of the risks of stroke and death due to endarterectomy for symptomatic carotid stenosis. Stroke. 1996;27:260-265.

7. Rothwell PM, Goldstein LB. Carotid endarterectomy for asymptomatic carotid stenosis: asymptomatic carotid surgery trial. Stroke. 2004;35:2425-2427.

8. Ross R. Atherosclerosis - An inflammatory disease. N Engl J Med. 1999;340:115-126.

9. Golledge J, Greenhalgh RM, Davies AH. The symptomatic carotid plaque. Stroke. 2000;31:774-781.

10. Falk E, Shah PK, Fuster V. Coronary plaque disruption. Circulation. 1995;92:657-671. 
11. Virmani R, Kolodgie FD, Burke AP, Farb A, Schwartz SM. Lessons from sudden coronary death: a comprehensive morphological classification scheme for atherosclerotic lesions. Arterioscler Thromb Vasc Biol. 2000;20:1262-1275.

12. Naghavi M, Libby P, Falk E, Casscells SW, Litovsky S, Rumberger J, Badimon JJ, Stefanadis C, Moreno P, Pasterkamp G, Fayad Z, Stone PH, Waxman S, Raggi P, Madjid M, Zarrabi A, Burke A, Yuan C, Fitzgerald PJ, Siscovick DS, de Korte CL, Aikawa M, Juhani Airaksinen KE, Assmann G, Becker CR, Chesebro JH, Farb A, Galis ZS, Jackson C, Jang IK, Koenig W, Lodder RA, March K, Demirovic J, Navab M, Priori SG, Rekhter MD, Bahr R, Grundy SM, Mehran R, Colombo A, Boerwinkle E, Ballantyne C, Insull W, Jr., Schwartz RS, Vogel R, Serruys PW, Hansson GK, Faxon DP, Kaul S, Drexler H, Greenland P, Muller JE, Virmani R, Ridker PM, Zipes DP, Shah PK, Willerson JT. From vulnerable plaque to vulnerable patient: a call for new definitions and risk assessment strategies: Part I. Circulation. 2003;108:1664-1672.

13. Hansson GK. Inflammation, atherosclerosis, and coronary artery disease. N Engl J Med. 2005;352:1685-1695.

14. Davies JR, Rudd JH, Weissberg PL, Narula J. Radionuclide imaging for the detection of inflammation in vulnerable plaques. J Am Coll Cardiol. 2006;47:C57-68.

15. Ben-Haim S, Israel O. PET/CT for atherosclerotic plaque imaging. $\mathrm{O} J$ Nucl Med Mol Imaging. 2006;50:53-60.

16. Okane K, Ibaraki M, Toyoshima H, Sugawara S, Takahashi K, Miura S, Shimosegawa E, Satomi J, Kitamura K, Satoh T. (18)F-FDG accumulation in atherosclerosis: use of $\mathrm{CT}$ and MR co-registration of thoracic and carotid arteries. Eur $\mathrm{J}$ Nucl Med Mol Imaging. 2006;33:589-594.

17. Wilhjelm JE, Gronholdt ML, Wiebe B, Jespersen SK, Hansen LK, Sillesen $\mathrm{H}$. Quantitative analysis of ultrasound B-mode images of carotid atherosclerotic plaque: correlation with visual classification and histological examination. IEEE Trans Med Imaging. 1998;17:910-922.

18. Montauban van Swijndregt $A D$, Elbers HR, Moll FL, de Letter J, Ackerstaff RG. Ultrasonographic characterization of carotid plaques. Ultrasound Med Biol. 1998;24:489-493. 
19. Wilensky RL, Song HK, Ferrari VA. Role of magnetic resonance and intravascular magnetic resonance in the detection of vulnerable plaques. J Am Coll Cardiol. 2006;47:C48-56.

20. MacNeill BD, Lowe HC, Takano M, Fuster V, Jang IK. Intravascular modalities for detection of vulnerable plaque: current status. Arterioscler Thromb Vasc Biol. 2003;23:1333-1342.

21. Larose E, Yeghiazarians Y, Libby P, Yucel EK, Aikawa M, Kacher DF, Aikawa E, Kinlay S, Schoen FJ, Selwyn AP, Ganz P. Characterization of human atherosclerotic plaques by intravascular magnetic resonance imaging. Circulation. 2005;112:2324-2331.

22. Stamper D, Weissman NJ, Brezinski M. Plaque characterization with optical coherence tomography. J Am Coll Cardiol. 2006;47:C69-79.

23. Schaar JA, van der Steen AF, Mastik F, Baldewsing RA, Serruys PW. Intravascular palpography for vulnerable plaque assessment. J Am Coll Cardiol. 2006;47:C86-91.

24. Madjid M, Willerson JT, Casscells SW. Intracoronary thermography for detection of high-risk vulnerable plaques. J Am Coll Cardiol. 2006;47:C80-85.

25. DeMaria AN, Narula J, Mahmud E, Tsimikas S. Imaging vulnerable plaque by ultrasound. J Am Coll Cardiol. 2006;47:C32-39.

26. Nicholls SJ, Tuzcu EM, Crowe T, Sipahi I, Schoenhagen P, Kapadia S, Hazen SL, Wun CC, Norton M, Ntanios F, Nissen SE. Relationship between cardiovascular risk factors and atherosclerotic disease burden measured by intravascular ultrasound. J Am Coll Cardiol. 2006;47:1967-1975.

27. Nicholls SJ, Sipahi I, Schoenhagen P, Crowe T, Tuzcu EM, Nissen SE. Application of intravascular ultrasound in anti-atherosclerotic drug development. Nat Rev Drug Discov. 2006;5:485-492.

28. Schoenhagen P, Tuzcu EM, Apperson-Hansen C, Wang C, Wolski K, Lin S, Sipahi I, Nicholls SJ, Magyar WA, Loyd A, Churchill T, Crowe T, Nissen SE. Determinants of arterial wall remodeling during lipidlowering therapy: serial intravascular ultrasound observations from the Reversal of Atherosclerosis with Aggressive Lipid Lowering Therapy (REVERSAL) trial. Circulation. 2006;113:2826-2834. 
29. Yuan C, Mitsumori LM, Beach KW, Maravilla KR. Carotid atherosclerotic plaque: noninvasive MR characterization and identification of vulnerable lesions. Radiology. 2001;221:285-299.

30. Fayad ZA, Fuster V. Clinical imaging of the high-risk or vulnerable atherosclerotic plaque. Circ Res. 2001;89:305-316.

31. Ruehm SG, Corot C, Vogt P, Kolb S, Debatin JF. Magnetic resonance imaging of atherosclerotic plaque with ultrasmall superparamagnetic particles of iron oxide in hyperlipidemic rabbits. Circulation. 2001;103:415-422.

32. Schmitz SA, Coupland SE, Gust R, Winterhalter S, Wagner S, Kresse M, Semmler W, Wolf KJ. Superparamagnetic iron oxide-enhanced MRI of atherosclerotic plaques in Watanabe hereditable hyperlipidemic rabbits. Invest Radiol. 2000;35:460-471. 


\section{Chapter \\ 2}

\section{In vivo detection of hemorrhage in human atherosclerotic plaques with Magnetic Resonance Imaging}

VC Cappendijk, KBJM Cleutjens, S Heeneman, GWH Schurink, RJThJ Welten, AGH Kessels, RJ van Suylen, MJAP Daemen, JMA van Engelshoven, ME Kooi

Published in JMRI 2004;20:105-110

This work was supported by a grant from the Dutch Heart Foundation (2000.173) 


\section{Abstract}

Purpose: To investigate the performance of high-resolution T1 weighted (T1w) turbo field echo (TFE) magnetic resonance imaging (MRI) for the identification of the high-risk component intra-plaque hemorrhage, which is described in the literature as a troublesome component to detect.

Materials and Methods: An MRI scan was performed pre-operatively on eleven patients who underwent carotid endarterectomy because of symptomatic carotid disease with a stenosis larger than $70 \%$. A common used double inversion recovery (DIR) T1 weighted turbo spin echo (TSE) served as T1 weighted control for the T1W TFE pulse sequence. The MR images were matched slice by slice with histology and the signal-to-noise ratio (SNR) and contrast-to-noise ratio (CNR) of the MR images were calculated. Additionally, two readers, who were blinded for the histological results, independently assessed the MR slices concerning the presence of intra-plaque hemorrhage.

Results: More than $80 \%$ of the histological proven intra-plaque hemorrhage could be detected using the TFE sequence with a high interobserver agreement $(\mathrm{Kappa}=0.73)$. The TFE sequence proved to be superior to the TSE sequence concerning SNR and CNR but also in the qualitative detection of intra-plaque hemorrhage. The false positive TFE results contained fibrous tissue and were all located outside the main plaque area.

Conclusion: The present study shows that in vivo high-resolution T1w TFE MRI can identify the high-risk component intra-plaque hemorrhage with a high detection rate in patients with symptomatic carotid disease. Larger clinical trials are warranted to investigate whether this technique can identify patients at risk for an ischemic attack. 


\section{Introduction}

The composition rather than the size of an atherosclerotic plaque has emerged as a predictor of plaques at risk for thrombo-embolic events. ${ }^{\prime}$ Advanced plaques with fibrous cap disruption and intra-plaque hemorrhage are largely responsible for morbidity and mortality ${ }^{2,3}$ and therefore in vivo non-invasive atherosclerotic intra-plaque hemorrhage visualization will be of invaluable importance to trace the vulnerable patient. However, there are no established diagnostic tools to visualize intra-plaque hemorrhage in vivo with sufficient accuracy. The spatial resolution of nuclear scintigraphy is too limited and a particular radiotracer is only able to identify one single component of complex atherosclerotic lesions. ${ }^{4}$ In vivo ultrasound plaque characterization lacks consistency and accuracy ${ }^{5,6}$ and is therefore not very useful for this purpose. Magnetic Resonance Imaging (MRI) is the most promising noninvasive method for in vivo plaque characterization, as stated in recent reviews ${ }^{7,8}$, and has already been found to detect the major plaque components in vivo, although accurate intra-plaque hemorrhage detection is still difficult. ${ }^{7,89}$ Cai et al $^{9}$ have reported a high sensitivity and specificity to identify type VI plaques (complex plaque with possible surface defect, hemorrhage or thrombus); however, this was based on the high accuracy of a time-of-flight MR pulse sequence to detect the thin or ruptured fibrous cap..$^{10,11,12}$ Additionally, Yuan et $\mathrm{al}^{10}$ described that acute hemorrhage had a high signal intensity in time-of-flight (gradient-echo) images, but the accuracy of acute hemorrhage detection could not be estimated due to a limited number of cases. Recently, Moody et $\mathrm{al}^{13}$ published about an accurate method to detect intra-plaque hemorrhage in the carotid artery of patients suffering anterior cerebral circulation ischemia using a T1 weighted (T1w) turbo field echo (TFE) sequence. This sequence is also referred to as a T1w magnetization-prepared three dimensional gradient echo sequence. High signal intensity identified histologically confirmed complicated plaque. However, the in-plane resolution was limited and no slice by slice comparison between MRI and histology was performed. A high in-plane resolution will be needed to detect small areas of intraplaque hemorrhage. The in-plane resolution can be improved considerably by using a small-diameter radio frequency surface coil. ${ }^{14}$ The purpose of 
the present study was to test the performance of a T1w TFE MR pulse sequence to detect intra-plaque hemorrhage in vivo with high resolution MRI. The MR slices will be compared on a slice by slice basis with histology. It will be investigated whether other tissue components than intra-plaque hemorrhage can also result in high signal intensity in the MR images. Because a T1w TSE sequence is a more commonly used T1w sequence for plaque characterization, we also obtained MR scans using this pulse sequence to compare the results.

\section{Materials and Methods}

\section{Subjects}

Eleven patients (mean age $=68 \pm 4$ years, range $=62-72$ years; 7 males) with a carotid stenosis of more than $70 \%$ as diagnosed by duplex ultrasound and scheduled to undergo carotid endarterectomy were recruited. All patients had suffered one or more transient ischemic attacks or minor strokes within 3 months before surgery. Before the operation an MRI scan was performed. The time interval between MRI scan and surgery was $5 \pm 4$ days (mean \pm standard deviation) with a range of 1-13 days. The institutional review committee approved the study and all subjects gave written informed consent.

\section{Histological Processing and Interpretation}

After surgery, the carotid endarterectomy plaques, which were removed in one piece, were marked laterally and ventrally in the longitudinal direction with two lines of ink of different colors. Thereafter, the specimens were formalin fixed, and subsequently, they were divided in $3 \mathrm{~mm}$ slices, processed, and embedded in paraffin. With on average 3-mm intervals a section of $4-\mu \mathrm{m}$ was subjected to histological hematoxylin-eosin staining. High quality color images were made of the histological slices. Two vascular biologists (K.C. and S.H.) microscopically assessed the histological slices concerning the major plaque components (fibrous tissue, lipid core, calcification and intra-plaque hemorrhage) in consensus and were unaware of the MRI results. The age of the intraplaque hemorrhage was categorized by two pathologists (M.D. and R.S.) 
as either younger or older than one week. Intra-plaque hemorrhage younger than one week was defined as the major component being intact and hemolyzed erythrocytes mixed with sleeves of fibrin with early signs of organization such as ingrowth of myofibroblasts and capillaries. Intraplaque hemorrhage older than one week was defined as the major component being granulation tissue, with or without hemosiderin pigment in macrophages, with remnants of intact and hemolyzed erythrocytes mixed with fibrin. Intra-plaque hemorrhage without signs of (early) organization was not taken into account because manipulation of the artery during operation resulting in mechanical damage of the arterial segment is most likely responsible for this recent intra-plaque hemorrhage. The size (in $\mathrm{mm}^{2}$ ) of all areas of intra-plaque hemorrhage was measured microscopically with a ruler.

\section{Matching Histology With MRI}

One investigator (V.C.C.) matched the histological and MRI slices. The carotid bifurcation was used as a landmark to match histology and MRI in the longitudinal direction of the artery. The cross-sectional shape of the plaque, together with the ink markings was used to adjust the crosssectional orientation of the slices.

\section{MRI Protocol}

The carotid artery was imaged using a 1.5 Tesla whole body MRI scanner (Intera, release 8.1.2, Philips Medical Systems, Best, The Netherlands). A dedicated, small-diameter, radio frequency surface coil with a diameter of $47 \mathrm{~mm}^{14}$ was fixed to the skin just above the carotid bifurcation. For an optimal scan result the patient was positioned in a head holder. An MR angiogram - balanced three-dimensional turbo field echo (TFE), scan parameters: repetition time (TR)/ echo time (TE) 4.4/2.1 ms, flip angle $60^{\circ}$, field of view (FOV) $200 \times 200 \mathrm{~mm}$, matrix size $192 \times 192$, number of signal averages $(\mathrm{NSA})=4$, slice thickness $=0.70 \mathrm{~mm}$, over contiguous transverse slices; body coil - was used for the identification of the carotid bifurcation.

Transverse images of the atherosclerotic plaques were obtained from about $7 \mathrm{~mm}$ caudal to $2 \mathrm{~cm}$ cranial of the bifurcation, a distance that covered the carotid plaque in all subjects. The following pulse sequences 
were acquired using the surface coil: 1) T1w three-dimensional TFE: TR/inversion time (TI)/TE 10.3/900/4.0 ms, flip angle 15०, 20 shots, actual scan percentage $=80 \%$, number of phase encoding steps per shot $163 / 20$, FOV $100 \times 100 \mathrm{~mm}$, matrix size $256 \times 205$ (in-plane resolution $=0.39 \times 0.49$ $\mathrm{mm}$ ), NSA = 6, 3.0-mm transverse slices; 2) T1w two-dimensional double inversion recovery (DIR) turbo spin echo (TSE) sequence: TR/TI/TE 570/255/14 ms, FOV 100×100 mm, matrix size 256×256 (in-plane resolution $=0.39 \times 0.39 \mathrm{~mm}$ ), NSA $=2,2.5-\mathrm{mm}$ transverse slices and $0.5-\mathrm{mm}$ slice gap. For both T1w TFE as for T1w TSE images a homogeneity correction was used to correct for the dropoff in sensitivity of the surface coil. The scan durations were 3:37 minutes and 7:05 minutes for nine slices for the T1w TFE and the T1w TSE sequence, respectively. Usually the T1w TSE MR images were obtained in two separate scans in order to reduce the scan duration of a single scan and thus to reduce motion artifacts.

\section{MR Image Evaluation}

All images were assessed on a Sun workstation using Easyscil (version 4.1.2, Philips Medical Systems, Best, The Netherlands). Signal-to-noise ratio (SNR) and contrast-to-noise ratio (CNR) for both the T1w TFE and the T1w TSE images were calculated. The signal was measured (V.C.C.) in an intra-plaque hemorrhage user defined region of interest (ROI). Noise was measured by taking the standard deviation of the signal in air outside the patient corrected for magnitude effects. ${ }^{15}$ Contrast was calculated as the difference between the signal intensity within an ROI of intra-plaque hemorrhage and that of fibrous tissue in plaque immediately adjacent to the intra-plaque hemorrhage area. One investigator (V.C.C.) indicated the ROIs, all with a clear match with the corresponding histological area with the same orientation in the plaque, on paper copies of the MRI slices. These indications on paper copies served only as an assurance that two readers assessed the same ROIs. The choice for this study design implies that the readers could not search the images randomly to identify areas of possible hemorrhage. These ROls were assessed independently by the two MR readers (J.v.E. and M.E.K.) on the workstation concerning plaque areas with signal intensities lower than, equal to or higher than that of surrounding muscle tissue (qualitative analysis). The area of muscle tissue considered was as close as possible to the ROI. High signal intensity was 
regarded as a positive test result for intra-plaque hemorrhage. The MR readers were not involved in patient enrollment in the study and had no access to the clinical notes and histological sections.

\section{Data Analysis}

Calculations were made with SPSS for Windows, version 10.0. Detection rates with 95\% confidence interval (Cl) for intra-plaque hemorrhage were calculated for the T1w TFE and the T1w TSE, respectively, using histology as standard of reference. Statistical difference in the performance reading the T1w TFE versus the T1w TSE images was tested with the McNemar Test. The agreement between the two readers was determined with Cohen's $\mathrm{K}$. The statistical difference of SNR and CNR between the two MR pulse sequences was investigated by paired-samples t-test.

\section{Results}

High-quality MR images could be obtained of all eleven patients. A total of $59 \mathrm{MRI}$ slices for both pulse sequences were available for comparison with histology. An average of $5 \mathrm{MRI}$ slices (range = 3-8) were obtained per patient. The vascular biologists scored 43 areas of intraplaque hemorrhage, 29 areas of fibrous tissue, 17 areas of calcification and 5 areas of lipid core in the corresponding 59 histological slices in mutual agreement. All 43 areas of intra-plaque hemorrhage were mainly younger than one week. A few of those 43 areas contained also very small areas of hemorrhage older than one week.

The SNR of the T1w TFE images was significantly better than that of the TSE images $(25 \pm 10$ (mean \pm standard deviation) versus $16 \pm 6$, respectively; $\mathrm{P}<0.01$, paired-samples t-test). Additionally the CNR between intra-plaque hemorrhage and fibrous tissue was significantly higher for the T1w TFE images (12 \pm 9 (mean \pm standard deviation) versus $4 \pm 3$, respectively; $\mathrm{P}<0.001$, paired-samples $\mathrm{t}$-test). The superior image quality of the T1w TFE images can be observed in Figure 1. 

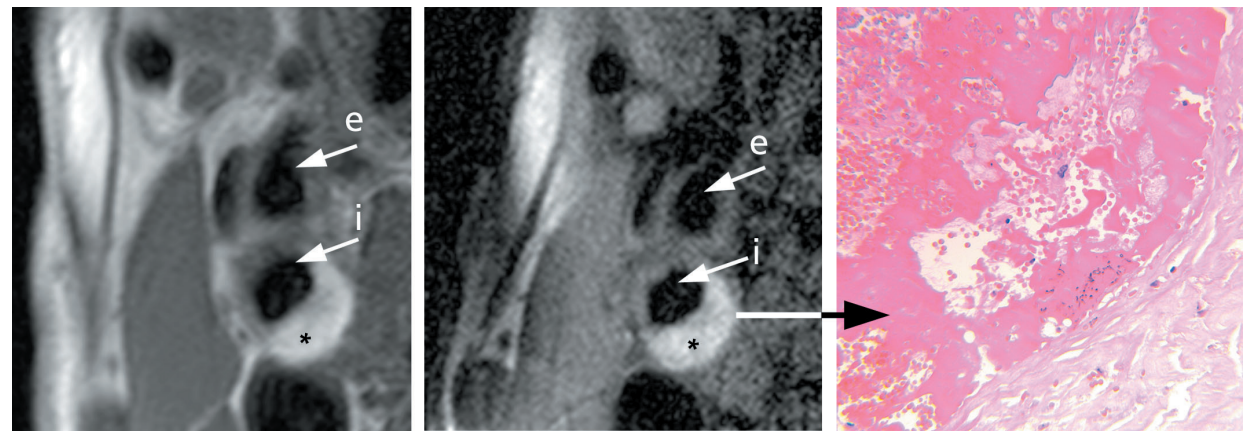

Figure 1. Left: T1w TFE image. Middle: T1w TSE image of the internal (arrow i) and external carotid artery (arrow e). Right: Magnification of corresponding histological hematoxylin-eosin stained slice (magnification, $\times 125)$. The large area of high signal intensity $\left(^{*}\right)$ in the internal carotid artery corresponds to intra-plaque hemorrhage, as shown by the histological slice.

Table 1. Concordance with histology and interobserver agreement of intra-plaque hemorrhage detection with high resolution MRI, using a T1w TFE sequence.

\begin{tabular}{|c|c|c|c|c|c|c|}
\hline \multicolumn{4}{|l|}{ Histology } & \multicolumn{3}{|c|}{ Histology } \\
\hline \multicolumn{4}{|c|}{ Intra-plaque hemorrhage } & \multicolumn{3}{|c|}{ No intra-plaque hemorrhage } \\
\hline \multicolumn{4}{|c|}{ Reader 2} & \multicolumn{3}{|c|}{ Reader 2} \\
\hline & & No & & & No & \\
\hline Reader 1 & Hemorrhage & hemorrhage & Total & Hemorrhage & hemorrhage & Total \\
\hline Hemorrhage & 35 & 0 & 35 & 4 & 0 & 4 \\
\hline \multicolumn{7}{|l|}{ No } \\
\hline hemorrhage & 5 & 3 & 8 & 8 & 39 & 47 \\
\hline Total & 40 & 3 & 43 & 12 & 39 & 51 \\
\hline
\end{tabular}

Table 2. Concordance with histology and interobserver agreement of intra-plaque hemorrhage detection with high resolution MRI, using a T1w TSE sequence.

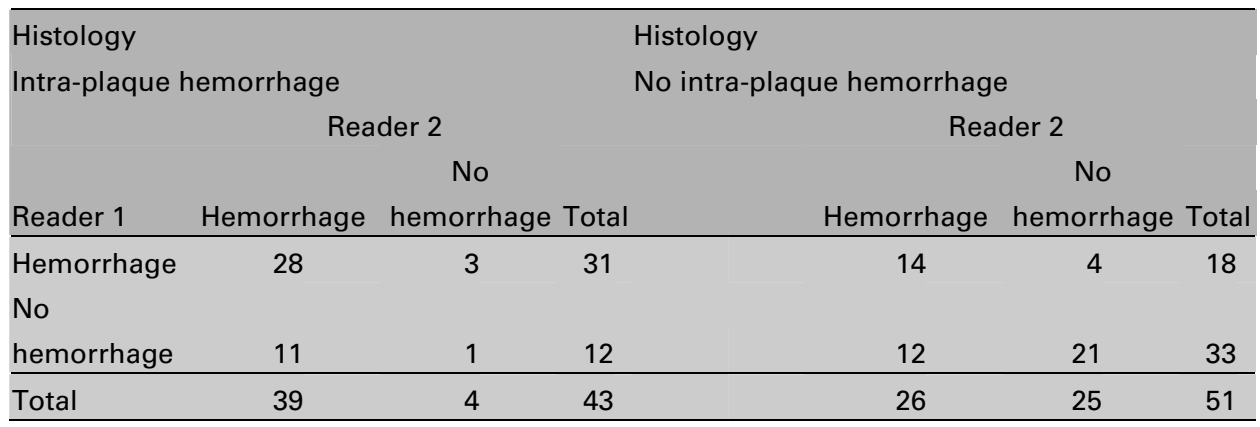


The results of both MRI readers for the T1w TFE sequence are summarized in Table 1 . Reader 1 (JvE) detected $81 \%(35 / 43 ; 95 \% \mathrm{Cl}=$ 67-92) and Reader 2 (MEK) 93\% (40/43; 95\% Cl = 81-99) of the areas of intra-plaque hemorrhage. There were 12 false positive (range $=1-5 \mathrm{~mm}^{2}$ ), 7 small false negative (range $=1-5 \mathrm{~mm}^{2}$ ), and one large false-negative $\left(11 \mathrm{~mm}^{2}\right)$ area for the two readers together. All false positive areas contained fibrous tissue and were located within the thickened vessel wall, but outside the main plaque area. The results of both readers for the T1w TSE sequence are presented in Table 2. Reader 1 detected 72\% (31/43; 95\% $\mathrm{Cl}=56-85)$ and reader $291 \%(39 / 43 ; 95 \% \mathrm{Cl}=78-97)$ of the areas of intraplaque hemorrhage. There were 30 false positive (range $=1-22 \mathrm{~mm}^{2}$ ) and 15 false negative $\left(1-11 \mathrm{~mm}^{2}\right)$ areas for both readers together. These false positive areas contained fibrous tissue $(n=23)$, lipid core $(n=4)$ and calcium $(n=3)$. The interobserver agreement was high for the TFE sequence (Kappa $=0.73 ; 95 \% \mathrm{Cl}=53-92)$, but low for the TSE sequence (Kappa $=0.35$, $95 \% \mathrm{Cl}=16-54)$. The areas of intra-plaque hemorrhage that were scored correctly by both readers on the TFE images had a size of $10 \pm 9 \mathrm{~mm}^{2}$ (mean \pm standard deviation, range $0.3-27 \mathrm{~mm}^{2}$ ) as measured in the histological slices. Both readers detected intra-plaque hemorrhage better with the TFE sequence, but this was not statistically significant (McNemar test). The TFE sequence is significantly better in excluding intra-plaque hemorrhage (McNemar test, $\mathrm{P}<0.005$ ).

\section{Discussion}

The present study demonstrates that in agreement with the recent study by Moody et $\mathrm{al}^{13}$, hemorrhage can be identified with a high detection rate and high interobserver agreement in atherosclerotic plaques of the carotid artery of patients with a recent history of transient ischemic attack or minor stroke with a commercially available state-of-the-art whole body MR scanner. More than $80 \%$ of the histological proven areas of intraplaque hemorrhage could be detected by both readers on the T1w TFE MR slices with a high interobserver agreement. Since plaques containing hemorrhage are considered to be at risk, ${ }^{1,2,3}$ this technique could be important in the selection of patients at risk for stroke. Additionally, in the present high-resolution study, small areas of hemorrhage could be 
detected. Furthermore, it was investigated whether other tissue components than hemorrhage could result in high signal intensity in the MR images as well. Finally, the performance of the T1w TFE sequence was compared both qualitatively and quantitatively with that of the T1w TSE sequence, which is more common used for plaque characterization.

The performance of the TFE sequence was superior to the TSE sequence, because the TFE sequence had a higher detection rate for intraplaque hemorrhage, a reasonable low number of false positive results, and a much better interobserver agreement. Additionally, the T1w TFE images had a significantly higher SNR and CNR, which resulted in a better image quality (Figure 1, page 24). Furthermore, another disadvantage of the TSE sequence was that it suffered more often from motion artifacts due to a longer scan duration. Both sequences have nearly the same inplane resolution.

False positive results might result in unnecessary carotid endarterectomy if intra-plaque hemorrhage is used as criterion to select the patients, and therefore they need to be very low in number. All false positive areas, i.e., areas of high signal intensity, represented fibrous tissue in the T1w TFE images (12/12), although fibrous tissue was in most cases iso- or hypo-intense signal (17/29). The false positive areas in the TFE sequence were all located within the thickened vessel wall, but outside the main plaque area, suggesting that it is important to interpret high MR signal intensity together with its location to prevent false positive results. Hemorrhage outside the main plaque area is rare and we observed it only once, which was scored incorrectly by both MR readers. The number of false positive results was much higher for the TSE images. One of the reasons for this higher number was that fibrous tissue far more often had a higher signal intensity in the TSE than in the TFE images (23/29 versus $12 / 29$, for the TSE and the TFE images respectively). Earlier it was postulated by $\mathrm{Xu}$ et $\mathrm{al}^{16}$ that fibrous tissue of an atherosclerotic plaque shows low signal intensity in gradient-echo images (TFE) because the layered collagen-rich structure of the cap reduces $T 2^{*}$. However, the signal intensity is high in a T1w spin echo pulse sequence (TSE) because the latter lacks a T2* effect. An example of a false positive area in a TSE MR image is given in Figure 2 (page 28). In this figure an area of fibrous tissue is hyperintense in the TSE MR image, but iso-intense in the TFE MR image. 
The most commonly accepted explanation for the high signal intensity of intra-plaque hemorrhage in T1w MR images is the presence of methemoglobin, a breakdown product of hemoglobin, which is formed about 12 hours after the hemorrhage. This produces a shortening of T1 and hence an increase in signal intensity in T1w images. Not much is known about the development over time of the MR signal intensity of hemorrhage in human atherosclerotic plaque. In swine, peak signal intensity in artificial luminal thrombi was observed after one week using a T1w spin echo sequence. ${ }^{17}$ The signal relapsed to normal in three weeks' time. It needs to be emphasized that the intra-plaque hemorrhage detected in the present study needs to be distinguished from the detection of luminal thrombi $i^{17,18}$ because from pathophysiological point of view luminal thrombi are quite different from intra-plaque hemorrhage. Intra-plaque hemorrhage can clearly be differentiated from luminal thrombus in histological slices. Bradley ${ }^{19}$ reported a very hyperintense signal in T1w MR images of parenchymal brain hematomas with an age between three and fourteen days. MR signal intensity of carotid intra-plaque hemorrhage also depends on age, as described by a number of groups. ${ }^{8,9,20,21}$ Another well recognized problem by these authors is partial-volume effects of small or inhomogeneous areas of hemorrhage. In the present study the false negative results were mainly small areas of less than $5 \mathrm{~mm}^{2}$.

The pathophysiology of advanced atherosclerotic disease is quite complex. Clot maturation in an atherosclerotic plaque may be impaired, and therefore the time elapsed between clot formation and full regeneration may vary considerably. ${ }^{22}$ Supplementary, capillaries, which invade the intra-plaque hemorrhage or atherosclerotic plaque thrombus within about 14 days $^{3,22}$, are easily damaged and are responsible for new spots of hemorrhage. One might assume that with the technique used in the present study only the existence of intra-plaque hemorrhage can possibly be predicted with MRI in vivo, especially the presence of methemoglobin, but not the age of the hemorrhage. This was supported with the histological observation that all areas with intra-plaque hemorrhage were less than one week old, while the mean delay between $\mathrm{MRI}$ and operation was five days. The mean delay and range was the same for the group of false negatives. The amount of methemoglobin will decrease in older areas of hemorrhage, and therefore, it is likely that in 
vivo MRI using a T1w TFE sequence will detect mainly young areas of intra-plaque hemorrhage.
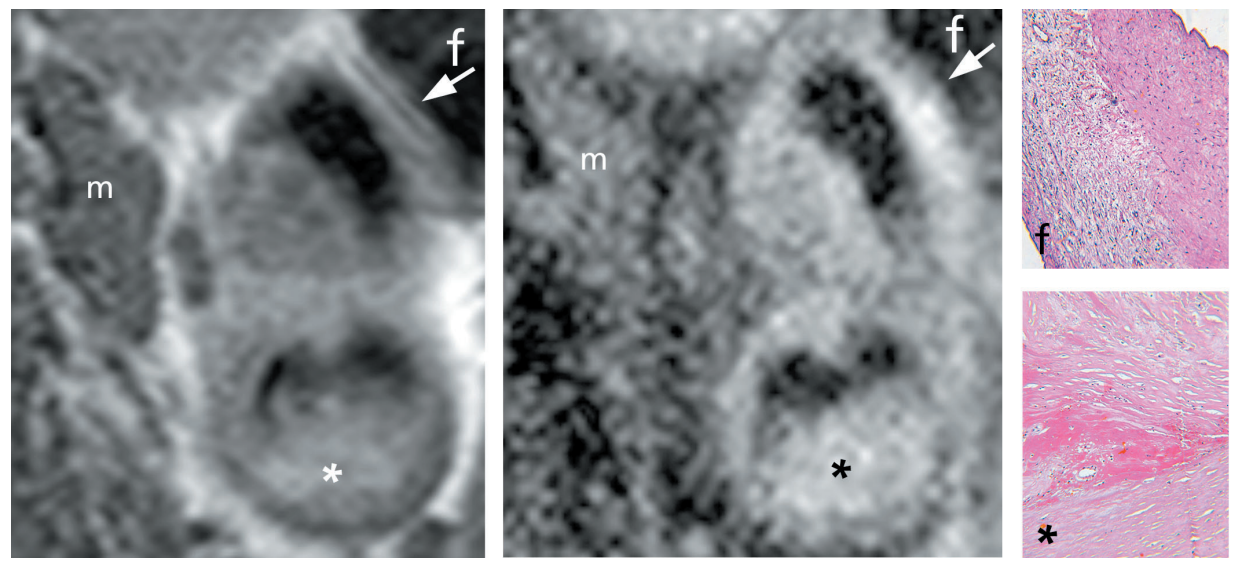

Figure 2. Example of a false positive T1w TSE result. Left: T1w TFE image. Middle: T1w TSE image. Right: Magnifications of corresponding histological hematoxylin-eosin stained slice (magnification, $\mathrm{x} 125$ ). Part of the carotid external artery appears with high signal intensity in T1w TSE, but not in T1w TFE (arrows). This area corresponds to fibrous tissue (f), as shown by the upper histological image. The area of high signal intensity $\left(^{*}\right)$ compared to muscle tissue $(\mathrm{m})$ in both T1w TFE and T1w TSE in the internal carotid artery corresponds to intraplaque hemorrhage, as shown by the lower histological image.

In the present study, no multisequence MRI was used since the objective was to investigate the performance of the T1w TFE sequence for hemorrhage detection. However, it needs to be emphasized that for an accurate separation of the other plaque components, multisequence MRI is essential. ${ }^{10,23,24}$

A limitation of the present study is that in order to be able to compare the performance of both MR readers, the ROls on the MR images could not be chosen randomly by the readers. This implies that the diagnostic performance might be somewhat biased. A second limitation is the limited number of subjects, which were included in the present investigation, and therefore, larger studies are warranted to confirm the current promising results. Additionally, it needs to be investigated whether patients with high signal intensity on the T1w TFE images are at risk for an ischemic attack.

In conclusion, the present study showed that non-invasive highresolution MRI, using a T1w TFE sequence, is very promising for the detection of intra-plaque hemorrhage. If the location of an area with high 
signal intensity in the vessel wall will be taken into account, the TFE sequence will be even more accurate. Because of both the much lower amount of false positive results, and the much higher interobserver agreement in TFE, this sequence will be more valuable for surgical decision-making than the TSE sequence. Future in vivo studies need to elucidate the time frame of sustained high signal intensity on T1w TFE images and its correlation with histology and patients' symptoms. The asymptomatic patients with high-grade stenosis are another challenging group that needs to be assessed.

\section{Acknowledgements}

This work was supported by a grant from the Dutch Heart Foundation (2000.173).

\section{References}

1. Falk $E$, Shah PK, Fuster V. Coronary plaque disruption. Circulation. 1995;92:657-671.

2. Virmani R, Kolodgie FD, Burke AP, Farb A, Schwartz SM. Lessons from sudden coronary death: a comprehensive morphological classification scheme for atherosclerotic lesions. Arterioscler Thromb Vasc Biol. 2000;20:1262-1275.

3. Van Damme $H$, Vivario $M$, Boniver J, Limet $R$. Histologic characterization of carotid plaques. Cardiovasc Pathol. 1994;3:9-17.

4. Manca G, Parenti G, Bellina R, Boni G, Grosso M, Bernini W, Palombo C, Paterni M, Pelosi G, Lanza M, Mazzuca N, Bianchi R, De Caterina R. ${ }^{111}$ In platelet scintigraphy for the noninvasive detection of carotid plaque thrombosis. Stroke. 2001;32:719-727.

5. Wilhjelm JE, Gronholdt ML, Wiebe B, Jespersen SK, Hansen LK, Sillesen $\mathrm{H}$. Quantitative analysis of ultrasound B-mode images of carotid atherosclerotic plaque: correlation with visual classification and histological examination. IEEE Trans Med Imaging. 1998;17:910-922. 
6. Montauban van Swijndregt $A D$, Elbers $H R$, Moll $F L$, de Letter J, Ackerstaff RG. Ultrasonographic characterization of carotid plaques. Ultrasound Med Biol. 1998;24:489-493.

7. Fayad ZA, Fuster V. Clinical imaging of the high-risk or vulnerable atherosclerotic plaque. Circ Res. 2001;89:305-316.

8. Yuan C, Mitsumori LM, Beach KW, Maravilla KR. Carotid atherosclerotic plaque: noninvasive MR characterization and identification of vulnerable lesions. Radiology. 2001;221:285-299.

9. Cai JM, Hatsukami TS, Ferguson MS, Small R, Polissar NL, Yuan C. Classification of human carotid atherosclerotic lesions with in vivo multicontrast magnetic resonance imaging. Circulation. 2002;106:13681373.

10. Yuan C, Mitsumori LM, Ferguson MS, Polissar NL, Echelard D, Ortiz G, Small R, Davies JW, Kerwin WS, Hatsukami TS. In vivo accuracy of multispectral magnetic resonance imaging for identifying lipid-rich necrotic cores and intraplaque hemorrhage in advanced human carotid plaques. Circulation. 2001;104:2051-2056.

11. Yuan C, Zhang SX, Polissar NL, Echelard D, Ortiz G, Davis JW, Ellington $E$, Ferguson MS, Hatsukami TS. Identification of fibrous cap rupture with magnetic resonance imaging is highly associated with recent transient ischemic attack or stroke. Circulation. 2002;105:181185.

12. Hatsukami TS, Ross R, Polissar NL, Yuan C. Visualization of fibrous cap thickness and rupture in human atherosclerotic carotid plaque in vivo with high-resolution magnetic resonance imaging. Circulation. 2000;102:959-964.

13. Moody AR, Murphy RE, Morgan PS, Martel AL, Delay GS, Allder S, MacSweeney ST, Tennant WG, Gladman J, Lowe J, Hunt BJ. Characterization of complicated carotid plaque with magnetic resonance direct thrombus imaging in patients with cerebral ischemia. Circulation. 2003;107:3047-3052.

14. Kooi ME, Cleutjens KBJM, Daemen MJAP, Kitslaar PJEHM, Kemering GJ, van Engelshoven JMA. In-vivo and in-vitro MRI of the carotid artery wall using a small diameter surface coil. Proc. Intl. Soc. Mag. Reson. Med. 2000;8:1657. 
15. Potchen EJ HE, Siebert JE, Gottschalk A. Magnetic Resonance Imaging. Concepts and Applications. St. Louis: Mosby; 1993.

16. Xu D, Ferguson MS, Hatsukami TS, Hwang JN, Yuan C. 3D gradient echo imaging of atherosclerotic plaque at human carotid artery: Quantitative evaluation of fibrous cap. Proc Intl. Soc. Mag. Reson. Med.; 2000.

17. Corti R, Osende JI, Fayad ZA, Fallon JT, Fuster V, Mizsei G, Dickstein E, Drayer B, Badimon JJ. In vivo noninvasive detection and age definition of arterial thrombus by MRI. Journal of the American College of Cardiology. 2002;39:1366-1373.

18. Johnstone MT, Botnar RM, Perez AS, Stewart R, Quist WC, Hamilton $J A$, Manning WJ. In vivo magnetic resonance imaging of experimental thrombosis in a rabbit model. Arterioscler Thromb Vasc Biol. 2001;21:1556-1560.

19. Bradley WG, Jr. MR appearance of hemorrhage in the brain. Radiology. 1993;189:15-26.

20. von Ingersleben G, Schmiedl UP, Hatsukami TS, Nelson JA, Subramaniam DS, Ferguson MS, Yuan C. Characterization of atherosclerotic plaques at the carotid bifurcation: correlation of highresolution MR imaging with histologic analysis-- preliminary study. Radiographics. 1997;17:1417-1423.

21. Coombs BD, Rapp JH, Ursell PC, Reilly LM, Saloner D. Structure of plaque at carotid bifurcation: high-resolution MRI with histological correlation. Stroke. 2001;32:2516-2521.

22. Leu HJ. [Histological age determination of arterial and venous thrombi and emboli]. Vasa. 1973;2:265-274.

23. Shinnar M, Fallon JT, Wehrli S, Levin M, Dalmacy D, Fayad ZA, Badimon JJ, Harrington $M$, Harrington $E$, Fuster $V$. The diagnostic accuracy of ex vivo MRI for human atherosclerotic plaque characterization. Arterioscler Thromb Vasc Biol. 1999;19:2756-2761.

24. Mitsumori LM, Hatsukami TS, Ferguson MS, Kerwin WS, Cai J, Yuan C. In vivo accuracy of multisequence MR imaging for identifying unstable fibrous caps in advanced human carotid plaques. J Magn Reson Imaging. 2003;17:410-420. 

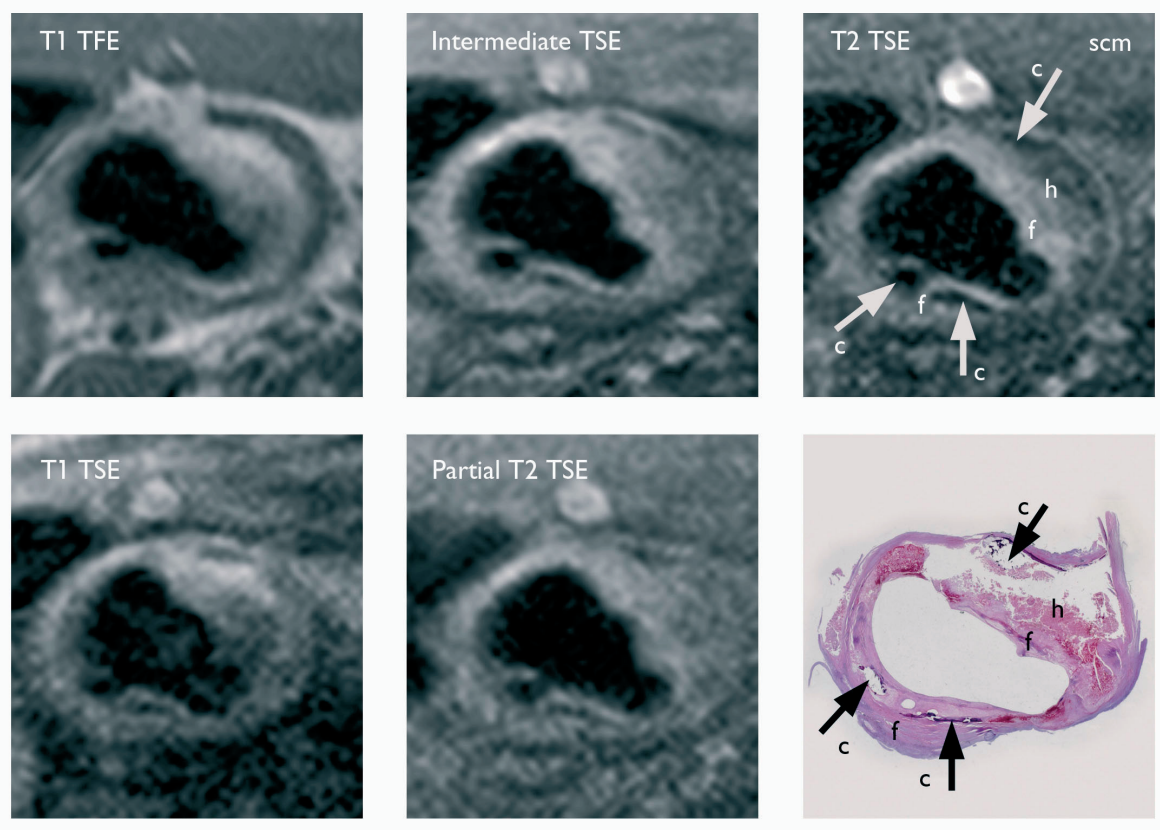

Figure 1, chapter 3. The transverse noncontrast enhanced images of five different MR pulse sequences (T1 weighted turbo field echo (T1 TFE), TR/TI/TE 10.3/900/4.0 ms, flip angle 15\%; Intermediate weighted turbo spin echo (TSE), ECG-gated, TR/TE 2RR/20 ms; T2 TSE, ECGgated, TR/TE 2 heartbeats (RR)/50 ms; T1 TSE, double inversion-recovery black blood technique, TR/TI/TE 570/255/14 ms; Partial T2 TSE, ECG-gated,_TR/TE 2RR/30 ms) on the same carotid artery level with corresponding hematoxylin-eosin stained histological section (magnification 12.5x). For the T1w TFE sequence, the in-plane resolution was $0.39 \times 0.49 \mathrm{~mm}$ with a slice thickness of $3.0 \mathrm{~mm}$ and no slice gap. For the other sequences, the in-plane resolution was $0.39 \times 0.39 \mathrm{~mm}$ with a slice thickness of $2.5 \mathrm{~mm}$ with a slice gap of $0.5 \mathrm{~mm}$. Signal intensity of plaque is compared to the signal intensity of the sternocleidomatoid muscle (scm). Abbreviations for tissue components are shown in the T2 weighted MR image and histological section. Hemorrhage (h) was well differentiated from fibrous (f) tissue (high and iso-intense relative signal intensity, respectively) on the T1w TFE image. On the T2 weighted TSE image fibrous (f) tissue has high signal intensity, while calcification (arrows) has very low signal intensity. Calcification (c) has very low relative signal intensity on Intermediate weighted TSE, T1 TSE and Partial T2 TSE as well. 


\section{Chapter}

\section{Assessment of human atherosclerotic carotid plaque components by multisequence MR imaging: Initial experience}

VC Cappendijk, KBJM Cleutjens, AGH Kessels, S Heeneman, GWH Schurink, RJThJ Welten, WH Mess, MJAP Daemen, JMA van Engelshoven, ME Kooi

Published in Radiology 2005;234:487-492

This work was supported by a grant from the Dutch Heart Foundation (2000.173). 


\section{Abstract}

Purpose: To prospectively determine, by using a stepwise logistic regression model, the optimal magnetic resonance (MR) weighting (i.e., pulse sequence) combinations for plaque assessment and corresponding cut-off values of relative signal intensities (rSI).

Materials and Methods: Institutional review board approval and patient consent was obtained. Eleven patients (mean age $68 \pm 4$ years; 7 males) with symptomatic carotid disease and stenosis of more than $70 \%$ were investigated at MRI before carotid endarterectomy. The MR images were matched with the histologic features of the endarterectomy specimens (reference standard). The rSls (compared to muscle tissue) from regions of interest were assessed qualitatively and semiquantitatively. For all major components (calcification, lipid core, intraplaque hemorrhage, and fibrous tissue), optimal cut-off points for the rSls were determined for five MR weightings by means of receiver operating characteristic curves. The best predicting combinations of these five dichotomized MR weightings were selected by means of stepwise logistic regression analysis. The potential sensitivity and specificity of MRI for vulnerable plaque with hemorrhage and/or lipid core were determined.

Results: The same optimal MR weighting combinations for identifying the four plaque components were found with qualitative and semiquantitative analysis. Sensitivity and specificity for vulnerable plaque were 93\% (95\% confidence interval: 77\%, 99\%) and 96\% (95\% confidence interval: $86 \%, 100 \%)$, respectively, for the qualitative analysis and $76 \%$ (95\% confidence interval: $56 \%, 90 \%$ ) and 100\% (95\% confidence interval: $93 \%, 100 \%$ ) for the semi-quantitative analysis.

Conclusion: The present study demonstrates the potential of a systematic approach of atherosclerotic plaque assessment with multisequence MRI by using the information provided by five different MR weightings in a stepwise logistic regression model. 


\section{Introduction}

The composition of an atherosclerotic plaque is an important predictor for thrombo-embolic events, ${ }^{1,2,3}$ and, intra-plaque hemorrhage and lipid core are considered as high-risk components. For the in vivo detection of these components, magnetic resonance imaging (MRI) with high spatial resolution has proved to be promising ${ }^{4,5}$. In particular, multisequence MRI is proposed for plaque characterization, since information from images obtained with multiple weightings (i.e., pulse sequences) has been shown to be superior to that from images all obtained with a single weighting. ${ }^{6,78}$ For instance, a combination of time of flight (TOF) and T1 weighted turbo spin echo (T1w TSE) images can help to differentiate intra-plaque hemorrhage and lipid core. Furthermore, resolving hemorrhage can be identified by combining intermediate weighted and T2w TSE images. ${ }^{9}$ However to our knowledge, no specific analysis of optimal MR weighting combinations has yet been performed. In addition, MR images are currently assessed qualitatively, by judging the relative signal intensity (rSI) of the various parts of plaque as lower than, equal to, or higher than that of surrounding muscle tissue. A semi-quantitative assessment might make plaque assessment more objective. Thus, the purpose of the present study was to prospectively determine, by using a stepwise logistic regression model, the optimal MR weighting combinations for plaque assesment and the corresponding cut-off values of rSls.

\section{Material and Methods}

\section{Subjects}

Eleven patients (mean age $68 \pm 4$ years, range $62-72$ years; 7 males) who had symptomatic carotid disease and were scheduled to undergo carotid endarterectomy were included consecutively in a period from October 2, 2001, to January 11, 2002. These eleven patients were all those who met the criteria during this period. All had carotid stenosis of more than $70 \%$, as diagnosed by duplex ultrasound. Symptomatic disease was defined as the occurrence of at least one transient ischemic attacks or minor strokes within the 3 months before surgery. MRI was performed 
$5 \pm 4$ days before surgery (mean \pm standard deviation) with a range of 1-13 days. The institutional medical ethical committee approved the study and all subjects gave written informed consent.

\section{$M R I$}

MR imaging was performed with a 1.5-T whole body imager (Philips Intera, release 8.1.2, Philips Medical Systems, Best, The Netherlands). For an optimal imaging result, the patient was positioned in a head holder. A dedicated small diameter radiofrequency surface coil with a diameter of $47 \mathrm{~mm}^{10}$ was fixed to the skin just above the carotid bifurcation. First, the carotid bifurcation was identified by means of MR angiography without contrast material enhancement (balanced 3D turbo field echo (TFE), body coil, scan parameters: repetition time (TR)/ echo time (TE) 4.4/2.1 ms, flip angle $60^{\circ}$, field of view (FOV) $200 \times 200 \mathrm{~mm}$, matrix size $192 \times 192$, number of signal averages (NSA) 4, slice thickness $0.70 \mathrm{~mm}$, overcontiguous transverse slices, scan duration 1:27 minutes).

Subsequently, nine transverse images with a slice thickness of $3 \mathrm{~mm}$ were obtained. Five MR weightings were used, as described in the next paragraph, from about $7 \mathrm{~mm}$ caudal to $2 \mathrm{~cm}$ cranial of the carotid bifurcation, so that the complete carotid plaque was imaged for all patients. Depending on the location of the carotid plaque, the surface coil was repositioned occasionally to achieve an optimal signal-to-noise ratio. The following five MR weightings were acquired after the coil was in its definitive position: I. Electrocardiography (ECG)-gated T2w turbo spinecho (TSE): TR/TE 2 heartbeats (RR)/50 ms, NSA 2, scan duration \pm 3:30 minutes; II. ECG-gated intermediate weighted TSE sequence (=proton density $w$ TSE, PDw TSE): TR/TE 2RR/20 ms, NSA 2, scan duration \pm 3:00 minutes; III. T1W 2D TSE sequence (double inversionrecovery black blood technique): TR/inversion time (TI)/TE 570/255/14 ms, NSA 2, scan duration 7:05 minutes. Usually, just five T1w TSE MR images were obtained around the bifurcation in order to reduce the scan duration and thus motion artifacts; IV. ECG-gated partial T2w TSE sequence: TR/TE 2RR/30 ms, NSA 4, scan duration \pm 1:30 minutes; $V$. T1 weighted (T1w) 3D TFE: TR/TI/TE 10.3/900/4.0 ms, flip angle $15^{\circ}, 24$ shots, actual scan percentage $80 \%$, number of phase encoding steps per shot $163 / 24$, NSA 6 , scan duration 3:37 minutes. For the T1w TFE sequence, the FOV was 
$100 \times 80 \mathrm{~mm}$ with a matrix size of 256x163 (in-plane resolution $0.39 \times 0.49 \mathrm{~mm}$ ) and a slice thickness of $3.0 \mathrm{~mm}$ and no gap between the slices. For the other sequences, the FOV was $100 \times 80 \mathrm{~mm}$ with a matrix size of 256x205 (in-plane resolution $0.39 \times 0.39 \mathrm{~mm}$ ) and a slice thickness of $2.5 \mathrm{~mm}$ with a slice gap of $0.5 \mathrm{~mm}$. Total investigation time was about 40 minutes.

\section{Histology}

Before the start of the study, the surgeons were trained to remove the carotid endarterectomy plaque in one piece. For each patient, this plaque served as the reference standard. The plaque was marked laterally and ventrally in the longitudinal direction with two lines of different-colored ink and then fixed with formalin. It was then divided into 3-mm transverse slices, processed, and embedded in paraffin, and 4- $\mu \mathrm{m}$ thick sections were subjected to hematoxylin-eosin and Elastica von Giesen staining.

The histological sections were studied microscopically. Color prints were made of the histological slices on which the major plaque components (fibrous tissue, lipid core, calcification and intra-plaque hemorrhage) were assigned in consensus by two vascular biologists (K.B.J.M.C., 5 years experience in vascular histology; S.H., 10 years experience in vascular histology) and a pathologist (M.J.A.P.D., 15 years experience in vascular histology). They were unaware of the MRI results.

\section{$M R$ images and corresponding histologic slices}

One investigator (V.C.C.) matched the MRI and histologic slices by using the carotid bifurcation or the narrowest lumen as landmark in the longitudinal orientation and the shape of the plaque and the ink markings to match the cross sectional orientation. The coregistered MRI and histologic slice at landmark level determined automatically the coregistration of the slices cranially and caudally from the landmark slice, since the cross-sectional orientation of these slices was determined from the ink markings, while the longitudinal position was determined from the known longitudinal distance between the slices. Subsequently, this investigator manually drew regions of interest (ROls) on the MR images (no more than three ROls per slice). ROls were included only if they clearly matched the histologic slices in size and shape and had a homogeneous 
appearance on the MR images. Their size depended on the area of the particular plaque component (mean $5.3 \mathrm{~mm}^{2} \pm$ mean $4.3 \mathrm{~mm}^{2}$; range, 0.6-22.1 $\mathrm{mm}^{2}$ ).

The relative signal intensities ( $\mathrm{rSI}$ ) of these ROls were assessed on a 4-point scale (much lower $[<<1]$, lower $[<1]$, equal $[=1]$ or higher $[>1]$ than the surrounding muscle tissue; qualitative assessment) by this investigator. The rSI of a $\mathrm{ROI}$ with an utter black appearance was judged as " $<<$ ", while a ROI with a grey appearance and a signal intensity lower than that of surrounding muscle tissue was judged as " $<$ ". Additionally, the absolute MR signal intensity (SI) in that ROI was measured and an rSI was calculated by dividing this $\mathrm{SI}$ by the $\mathrm{SI}$ measured in surrounding muscle tissue (semi-quantitative assessment; Easyscil, version 4.1.2, Philips Medical Systems, Best, The Netherlands). The location of the ROI was registered as either within the main plaque or within the thickened vessel wall for both the qualitative and semi-quantitative assessment.

\section{Logistic regression model}

The four tissue components were distinguished in three steps by using ROC curve analysis. ${ }^{11}$ In every step one component was differentiated from the remaining components. The choice for the order of the three steps was determined by the ranking of the mean $\mathrm{rSI}$ of the four tissue components. In the first step, calcification, which had the lowest mean rSI in all MR weightings, was differentiated from the other components. In the second step lipid core, which had the second lowest mean $\mathrm{rSI}$ in all MR weightings, was differentiated from fibrous tissue and intra-plaque hemorrhage, and in the final step intra-plaque hemorrhage was differentiated from fibrous tissue. In every step, the optimal cut-off rSI values were determined for all five MR weightings to be able to dichotomize these five MR weightings. The cut-off values were based on the minimal distance to the upper left corner of the ROC curve. The location of the ROI (in the main plaque or in the thickened vessel wall) was an additional binary parameter in the third step. In each step of the model, stepwise logistic regression analysis was performed with subsequent ROC curve analysis to find the combination of MR weightings which differentiated best between the component of interest and the remaining components. ${ }^{12}$ After completion of the three steps and the prediction of all 
four components, the potential detection rate of the individual components and sensitivity and specificity for the MR identification of the vulnerable plaque were calculated. The vulnerable plaque was defined as plaque containing regions of intra-plaque hemorrhage and / or lipid core. The sensitivities and specificities of the qualitative and semi-quantitative $\mathrm{rSI}$ assessments for the presence of intra-plaque hemorrhage/lipid core were compared with a McNemar Test. Because for each patient multiple locations were used for data analysis, the ROls were assessed for codependence by calculating the Pearson correlation between the rSls of adjoining ROls for each MR sequence.

All statistical analysis were performed with SPSS 10.0, Chicago, IL, for Windows '98.

\section{Results}

A total of 63 MR slices and corresponding histological slices from eleven patients were assessed (each MR slice should consist of five MR weightings $\rightarrow 5 \mathrm{MR}$ weightings $\times 63=315$ images). A mean of 5.7 slices (range 4-8) were obtained per patient. An example of a set of five corresponding MR weightings and the histological section is presented in Figure 1 (page 32). Seventy-two of the $315 \mathrm{MR}$ images could not be analyzed because they were missing $(n=48)$ or had an artifact $(n=24)$. The missing MR images were predominantly located on the upper or lower slice levels because the patient moved slightly in longitudinal direction or because the MR weighting was not acquired on that particular slice level to reduce scan time. The MR images with artifacts and the missing MR images were equally distributed over the five MR weightings, except for T1w TFE, which scored obvious better with just 6 of 63 non-assessable MR slices (Table 1). Ninety-nine regions of homogeneous tissue were assigned on the 63 histological color images as calcification, lipid core, intra-plaque hemorrhage and fibrous tissue in 18, 8, 30 and 43 occasions, respectively. The stepwise logistic regression analysis on the 243 available MR images resulted in the MR prediction of 79 of these 99 regions of homogeneous tissue. For 20 of 99 regions there was no MR weighting combination available for both the qualitative and semi-quantitative rSI analysis. 
The mean rSI of each component and corresponding MR weighting is shown in Figure 2. The pattern of rSls is similar for the qualitative and semi-quantitative data. The number of measurements for each component and MR weighting were based on the numbers listed in Table 1.

Table 1. Number of MR slices (N) available for each MR weighting and the number of ROls on which measurements were performed.

\begin{tabular}{|c|c|c|c|c|c|}
\hline MRI & $\begin{array}{l}\text { T1w } \\
\text { TFE }\end{array}$ & $\begin{array}{l}\text { T2w } \\
\text { TSE }\end{array}$ & $\begin{array}{c}\text { Intermediate } \\
\text { TSE* }^{*}\end{array}$ & $\begin{array}{l}\text { T1w } \\
\text { TSE }\end{array}$ & $\begin{array}{c}\text { Partial T2w } \\
\text { TSE }\end{array}$ \\
\hline $\begin{array}{l}\mathrm{N}=63 \text { slices } \\
\text { Histology }\end{array}$ & $\mathrm{N}=57$ & $\mathrm{~N}=44$ & $N=48$ & $N=48$ & $\mathrm{~N}=46$ \\
\hline (99 ROls) & & & & & \\
\hline $\begin{array}{l}\text { Calcification } \\
\text { (18) }\end{array}$ & 13 & 11 & 12 & 16 & 12 \\
\hline $\begin{array}{l}\text { Lipid core } \\
\text { (8) }\end{array}$ & 7 & 6 & 6 & 5 & 6 \\
\hline $\begin{array}{l}\text { Hemorrhage } \\
(30)\end{array}$ & 30 & 23 & 26 & 23 & 24 \\
\hline $\begin{array}{l}\text { Fibrous } \\
\text { (43) }\end{array}$ & 40 & 33 & 35 & 36 & 35 \\
\hline
\end{tabular}

* Intermediate TSE = proton density TSE (PDw TSE)

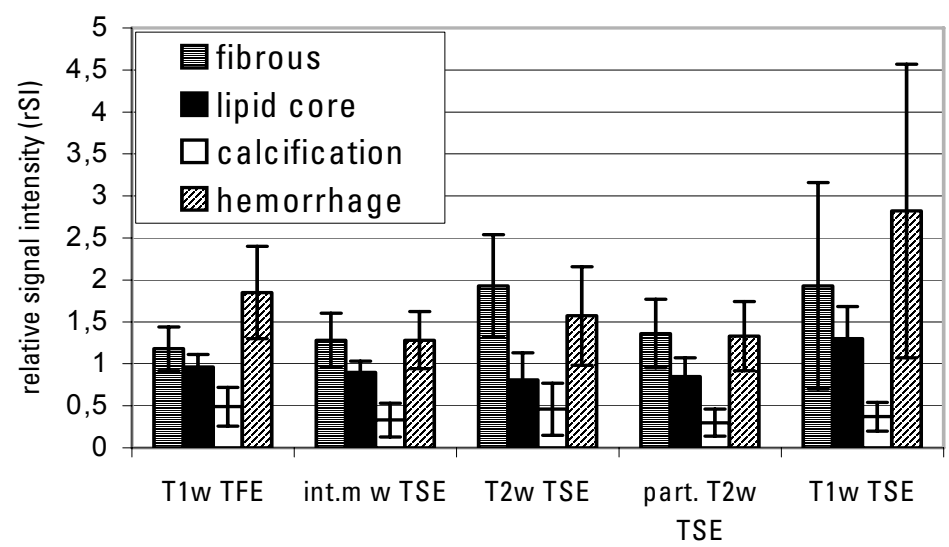

Figure 2. Descriptive of mean and standard deviation of relative signal intensities (rSI) for 99 regions of atherosclerotic plaque tissue is shown in the histogram (semi-quantitative data) to illustrate the differences in rSls of the plaque components for the five different MR weightings. The top of the bars indicates the mean $\mathrm{rSI}$ and the vertical lines represent the standard deviation. The histogram for qualitative data has a similar pattern (not shown). The histogram was based on the numbers listed in Table 1. 


\section{Qualitative and semi-quantitative analysis}

The optimal MR weighting combinations and the determined cut-off values together with the detection rates for the four plaque components, for both the qualitative and semi-quantitative rSI analysis, are listed in Figure 3. The T2w TSE and T1w TFE weighting were essential for plaque assessment while a random combination of two out_of the partial T2w TSE, T1W TSE and intermediate weighted TSE was required for the identification of calcification. The qualitative and semi-quantitative detection rates for calcification, lipid core, intra-plaque hemorrhage and fibrous tissue were, respectively, $18 / 18(100 \%)$ and 18/18 (100\%), $6 / 6(100 \%)$ and $5 / 6(83 \%), 21 / 23(91 \%)$ and $17 / 23(74 \%), 32 / 32(100 \%)$ and $30 / 32(94 \%)$ (Figure 3). Sensitivity and specificity and $95 \%$ confidential intervals, which can potentially be reached, were calculated for plaques containing intra-plaque hemorrhage and/or lipid core (Table 2). The qualitative analysis showed a significant better sensitivity $(P<0.05$, McNemar Test) for the detection of plaques containing intra-plaque hemorrhage/lipid core (Table 2), but this is at cost of specificity.

Table 2. Sensitivities and specificities (95\% confidential interval) for carotid atherosclerotic plaques containing intra-plaque hemorrhage and/or lipid core for the qualitative and semiquantitative analysis. Seventy-nine regions of interest were predicted by the logistic regression model.

\begin{tabular}{lcr}
\hline & $\begin{array}{c}\text { Sensitivity } \\
\text { (ratio, 95\% C.I.) }\end{array}$ & $\begin{array}{c}\text { Specificity } \\
\text { (ratio, 95\% C.I.) }\end{array}$ \\
\hline Qualitative assessment of rSls* & $93 \%,(27 / 29,77-99)$ & $96 \%,(48 / 50,86-100)$ \\
Semi-quantitative assessment of rSls* & $76 \%,(22 / 29,56-90)$ & $100 \%,(50 / 50,93-100)$ \\
\hline
\end{tabular}

${ }^{*}$ rSls $=$ relative signal intensities

For 20 of 99 ROls there was no MR weighting combination available, because of the seventy-two MR images which could not be analyzed. Replacement of the selected MR weighting combinations by the best single available MR weighting resulted in erroneous prediction of 8 of 20 and 10 of 20 ROls for the qualitative as well as for the semi-quantitative assessment, respectively. If a T2w TSE image was missing and replaced by other single MR weightings then this was responsible for 6 of 8 and 7 of 10 errors for the qualitative and semi-quantitative analysis, respectively. 
Qualitative

analysis
Semi-quantitative

analysis

Step I

\begin{tabular}{|c|c|c|c|c|}
\hline \multicolumn{2}{|c|}{ a random combination of } & \multirow[b]{3}{*}{ Calcification (18 ROIs) } & \multicolumn{2}{|c|}{ a random combination of } \\
\hline (int.m. w TSE & $<<1)$ & & (Int.m. w TSE & $<0.71)$ \\
\hline (Partial T2w TSE & $<<1)$ & & (Partial T2w TSE & $<0.51)$ \\
\hline (T1w TSE & $<<1)$ & & (T1w TSE & $<0.64)$ \\
\hline (T2w TSE & $<<1) \quad 18$ & & 18 & \\
\hline
\end{tabular}
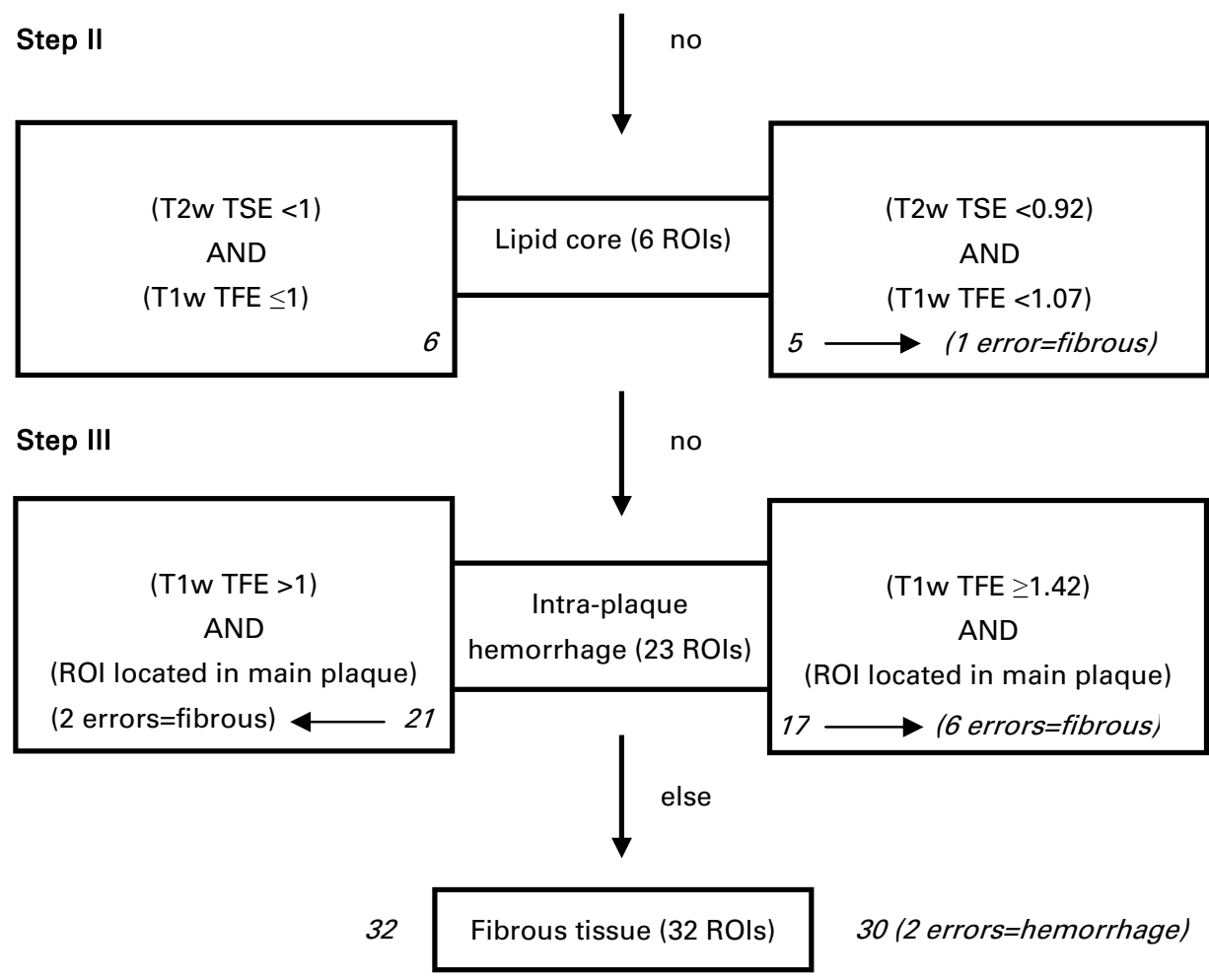

Figure 3. The MR weighting combinations and their relative signal intensity (rSI) cut-off values for the qualitative (big boxes on the left) and semi-quantitative (big boxes on the right) prediction of calcification, lipid core, intra-plaque hemorrhage and fibrous tissue (79 ROIs). The detection rate for a component is the ratio of correct predicted ROIs (in italic) and the total number of ROIs of that component. Int.m. $w$ = Intermediate weighted. (= Proton density weighted, PDw) 
Totally, in the present study the T2w TSE MR slice was missing in 26 ROIs, in 9 occasions because of a motion artifact and in 17 occasions because the image was missing on a certain slice level.

The correlation between the rSls of adjoining ROls was low $(r<0.4)$ for all MR sequences except for the T1w TSE sequence for which the correlation was high $(r=0.76)$. Note that the T1w TSE sequence was not an essential sequence for plaque assessment. For the other sequences each location could be considered as an independent assessment.

\section{Discussion}

The present study demonstrates the potential of a systematic approach of atherosclerotic plaque assessment with multisequence MRI using the information provided by five different MR weightings in a stepwise logistic regression model. This study shows the optimal MR weighting combinations of the five tested MR weightings for the detection of the various atherosclerotic plaque components. Furthermore, not only qualitative but also semi-quantitative analysis was performed.

It appeared that the combination of the T2w TSE and T1w TFE MR weighting was essential for the differentiation between the four major tissue components. A complete scan-protocol to assess a patient with atherosclerotic carotid disease needed these two MR weightings and a random combination of two out of Partial T2w TSE, T1w TSE or intermediate weighted TSE. We recommend to choose the T1w TSE and intermediate weighted TSE sequence since these sequences are most distinct from the other sequences. The attainable sensitivity and specificity in identifying vulnerable plaques with hemorrhage and/or lipid core were comparable to previous reported values.' The detection rate of each individual component was above $80 \%$ for both the qualitative as the semiquantitative analysis, except for the semi-quantitative detection rate of intra-plaque hemorrhage, which was $74 \%$.

If the information of single MR weightings was used, this resulted in a wrong prediction of a $\mathrm{ROI}$ in about one of every two ROIs. Therefore, in case a motion artifact occurs in one of the images from the chosen scanprotocol the involved MR weighting should be repeated. Additionally, it is 
also important to plan all the different MR weightings exactly similar around the carotid bifurcation.

Qualitative as well as semi-quantitative parameters of rSls for plaque characterization have been determined. There was no statistically significant difference in accuracy between the qualitative and semiquantitative plaque assessment and the selected $M R$ weighting combinations were similar for each component. For clinical use, however, the semi-quantitative assessment of plaque components is too timeconsuming and this makes computerized assessment techniques necessary.

A possible limitation of the present study is that no time of flight (TOF) MR weighting was included. Previously, it has been shown that this sequence can be used for the visualization of the fibrous cap. ${ }^{13}$ Additionally, by using a bright-blood technique the lumen can be distinguished from calcified plaque adjoining the lumen. ${ }^{7}$ In the present population, the absence of the TOF weighting did not seem to matter since in all cases calcification was identified by MRI and the detection rate of fibrous tissue was $94 \%$. Currently, we showed that calcification could be identified by using a random pair of a Partial T2w TSE, T1w TSE or intermediate weighted TSE. Possibly, in future studies, one of these sequences combined with a TOF sequence could be used instead. The added value of a TOF MR weighting or other newly proposed MR weightings could be tested using the systematic approach described in the present study. Another shortcoming of the present study is that the selection of plaque components by MR weighting combinations and the calculation of sensitivity and specificity for these components was performed on the same data set and that MRI and histology were matched before measurements. As a consequence, the level of sensitivities and specificities is the highest potentially achievable. Therefore, the algorithm of MR weighting combinations and their corresponding cut-off points needs to be tested on a new series of patients without the knowledge of the available histology. This is important because the non-invasive diagnosis of the vulnerable plaques may become extremely valuable for future clinical trials assessing the vulnerable atherosclerotic patient. ${ }^{14}$ Additionally, for each patient multiple locations were used for data analysis. Although the correlation between the rSls of adjoining ROls was 
low $(r<0.4)$ for the essential MR sequences, this could result in wider confidence levels of sensitivity and specificity than those noted. Finally, a general limitation of studies in which MR images are compared with histology is the potential image misregistration between histology and MRI. In addition to methods previously described ${ }^{8,13}$ to perform the coregistration as accurately as possible, the plaque was marked laterally and ventrally in the longitudinal direction with two lines of ink of different colors. These two lines facilitated coregistration of the cross-sectional orientation considerably.

In conclusion, optimal MR weighting combinations of five MR weightings were found by a stepwise logistic regression analysis. Using these combined weightings, high spatial resolution MRI could qualitatively and semi-quantitatively characterize the vulnerable atherosclerotic plaque containing intra-plaque hemorrhage and/or lipid core with potentially a high sensitivity and specificity. These results, however, have to be proven with larger scale prospective studies. 


\section{References}

1. Falk $E$, Shah PK, Fuster V. Coronary plaque disruption. Circulation 1995; 92:657-671.

2. Virmani R, Kolodgie FD, Burke AP, Farb A, Schwartz SM. Lessons from sudden coronary death: a comprehensive morphological classification scheme for atherosclerotic lesions. Arterioscler Thromb Vasc Biol 2000; 20:1262-1275.

3. Van Damme $H$, Vivario $M$, Boniver J, Limet $R$. Histologic characterization of carotid plaques. Cardiovasc Pathol 1994; 3:9-17.

4. Fayad ZA, Fuster V. Clinical imaging of the high-risk or vulnerable atherosclerotic plaque. Circ Res 2001; 89:305-316.

5. Yuan C, Mitsumori LM, Beach KW, Maravilla KR. Carotid atherosclerotic plaque: noninvasive MR characterization and identification of vulnerable lesions. Radiology 2001; 221:285-299.

6. Shinnar M, Fallon JT, Wehrli S, et al. The diagnostic accuracy of ex vivo MRI for human atherosclerotic plaque characterization. Arterioscler Thromb Vasc Biol 1999; 19:2756-2761.

7. Yuan C, Mitsumori LM, Ferguson MS, et al. In vivo accuracy of multispectral magnetic resonance imaging for identifying lipid-rich necrotic cores and intraplaque hemorrhage in advanced human carotid plaques. Circulation 2001; 104:2051-2056.

8. Mitsumori LM, Hatsukami TS, Ferguson MS, Kerwin WS, Cai J, Yuan C. In vivo accuracy of multisequence MR imaging for identifying unstable fibrous caps in advanced human carotid plaques. J Magn Reson Imaging 2003; 17:410-420.

9. O'Brien KD, Cai J, Kampschulte A, et al. Hyperintense signals on T2and Proton Density-weighted magnetic resonance images identify regions of resolving hemorrhage in human carotid atherosclerosis. Abstract American Heart Association-Scientific Sessions 2002. Chicago, 2002; Circulation 2002; 106:II-1.

10. Kooi ME, Cappendijk VC, Cleutjens KB, et al. Accumulation of ultrasmall superparamagnetic particles of iron oxide in human atherosclerotic plaques can be detected by in vivo magnetic resonance imaging. Circulation 2003; 107:2453-2458. 
11. Hanley JA, McNeil BJ. The meaning and use of the area under a receiver operating characteristic (ROC) curve. Radiology 1982;143:29-36

12. Hosmer DW, Lemeshow S. Assessing the fit of the model, In: Cressie NAC, ed. Applied Logistic Regression. New York, NY: Wiley Interscience, 2000; 143-202.

13. Hatsukami TS, Ross R, Polissar NL, Yuan C. Visualization of fibrous cap thickness and rupture in human atherosclerotic carotid plaque in vivo with high-resolution magnetic resonance imaging. Circulation 2000; 102:959-964.

14. Naghavi $M$, Libby $P$, Falk $E$, et al. From vulnerable plaque to vulnerable patient: a call for new definitions and risk assessment strategies: Part I. Circulation 2003; 108:1664-1672. 


\section{Chapter \\ 4}

\section{In vivo MR quantification of the lipid-rich necrotic core in carotid atherosclerotic plaque}

VC Cappendijk, S Heeneman, AGH Kessels, KBJM Cleutjens, GWH Schurink, RJThJ Welten, WH Mess, RJ van Suylen, T Leiner, MJAP Daemen, JMA van Engelshoven, ME Kooi

Submitted to European Radiology

This work was supported by a grant from the Dutch Heart Foundation (2000.173). 


\section{Abstract}

Introduction: A large lipid-rich necrotic core (LRNC) is regarded as one of the key indicators of atherosclerotic plaque vulnerability. However, the amount of LRNC associated with increased risk for thrombo-embolic events is unknown and therefore quantification may aid better risk assessment. The aim of the current study was to determine the accuracy of quantification of the relative amount of LRNC in carotid atherosclerotic plaques with MRI.

Materials and Methods: Sixty-four symptomatic patients with carotid stenosis $\geq 70 \%$ were included. Multisequence MRI was performed shortly before endarterectomy. Two MRI readers classified relative signal intensities in regions of interest in the vessel wall around the carotid bifurcation. The relative amount of LRNC was quantified using an algorithm based on fixed combinations of multiple MR pulse sequences as well as solely based on T1w turbo field echo (TFE) images. Interreader agreement was expressed by intraclass correlation coefficients (ICC). Histology served as reference standard. Agreement between MRI and histology was determined by linear regression analysis.

Results: Interreader reproducibility for quantification of LRNC based on multi- as well as single sequence (T1w TFE) MRI was high (ICC, 95\% Cl): $0.86(0.77-0.94)$ and 0.91 (0.85-0.95), respectively. Good agreement between MRI and histology was found for both MR readers for quantification based on multisequence as well as single sequence MRI, with linear regression coefficients $\geq 0.80$ ( $p<0.0001)$.

Conclusion: The amount of LRNC as assessed by single sequence T1w TFE MRI is a reproducible, accurate, and fast way to quantify LRNC in vivo in carotid atherosclerotic plaque. 


\section{Introduction}

Rupture of atherosclerotic plaques is the predominant underlying process in the pathogenesis of acute cardiovascular syndromes like myocardial infarction or stroke. It is now widely accepted that plaque vulnerability is primarily determined by plaque composition as opposed to the degree of luminal narrowing. ${ }^{1,2}$ Although large randomized clinical trials in patients with carotid artery atherosclerosis ${ }^{3,4}$ and additional analysis of these trials ${ }^{5,6,7}$ found a clear benefit of endarterectomy for symptomatic patients with a carotid stenosis $>70 \%$, the vast majority $(80 \%)$ of these patients on medical treatment alone will not suffer a stroke within three years. In addition, the benefit of surgery for asymptomatic patients with a high-grade stenosis was found to be small. ${ }^{8}$ In light of these findings it is clear that additional parameters for the assessment of risk for thrombo-embolic complications beyond the degree of luminal narrowing alone would be extremely valuable. The presence of a large lipid-rich necrotic core (LRNC) is regarded as one of the key indicators of plaque vulnerability..$^{9,10,11}$ Additionally, it has been suggested ${ }^{12}$ that the presence of hemorrhage in the LRNC may be a stimulus for growth of the LRNC. Present thinking is that a larger LRNC confers a higher risk for thromboembolic complications. However, the amount of LRNC associated with an increased risk for thrombo-embolic events is unknown. Quantification of the amount of LRNC could therefore be an important tool to improve risk assessment.

Currently, magnetic resonance imaging (MRI) is one of the most promising tools for atherosclerotic plaque imaging and several authors demonstrated the capability of MRI to identify various plaque

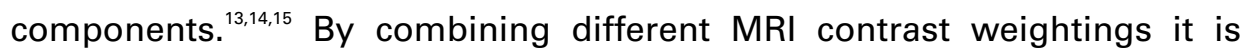
possible to discriminate different tissue types present in plaques. Cappendijk et $\mathrm{al}^{16}$ recently developed an algorithm for visual plaque assessment, based on signal intensities of plaque components at different MR pulse sequences. They ${ }^{16}$ determined the optimal combination of five MR pulse sequences to detect the various plaque components and found high sensitivity $(93 \%)$ and specificity $(96 \%)$ for the presence of the LRNC. Moody et $\mathrm{al}^{17}$, on the other hand, using only a single T1 weighted (w) magnetization prepared three-dimensional gradient echo MR sequence 
also found high sensitivity and specificity of $84 \%$, for the identification of the LRNC with hemorrhage.

The purpose of the present study was to prospectively assess the accuracy of the algorithm of Cappendijk et $\mathrm{al}^{16}$ as well as T1 $\mathrm{w}$ turbo field echo (TFE) imaging only for quantification of the relative amount of LRNC in atherosclerotic carotid plaque. Histology served as the reference standard.

\section{Materials and Methods}

\section{Subjects}

The institutional medical ethics committee approved the study and all subjects were required to give written informed consent prior to inclusion.

Inclusion criteria were one or more ipsilateral transient ischemic attacks (TIA) or minor strokes within three months before surgery, an MRI examination within 2 weeks prior to endarterectomy and the availability of a complete surgical specimen. MR images without corresponding histologic slices were excluded from analysis.

In general, in our hospital patients with a carotid stenosis $>70 \%$ but without TIA or stroke are not operated on.

\section{$M R I$}

MRI examinations were performed with a $1.5 \mathrm{~T}$ whole body MRI scanner (Philips Intera, release 8.1.2, Philips Medical Systems, Best, The Netherlands). A commercially available radio frequency surface coil with a diameter of $47 \mathrm{~mm}$ (Philips Medical Systems, Best, The Netherlands) was placed over the skin just above the carotid bifurcation. After location of the carotid bifurcation, a standardized multisequence MRI protocol consisting of T1, T2, Partial T2, Proton Density (PD) weighted (w) turbo spin echo (TSE) and T1w turbo field echo (TFE) sequences was used as described previously. ${ }^{16}$

In brief, these five MR pulse sequences were obtained with the following parameters: electrocardiography (ECG)-gated T2w TSE: repetition time (TR)/echo time (TE) 2 heartbeats (RR)/50 ms, number of signal averages (NSA) 2, echo train length 8; ECG-gated PDw TSE 
sequence: TR/TE 2RR/20 ms, NSA 2, echo train length 5; T1w TSE (double inversion-recovery black blood technique): TR/inversion time (TI)/TE 570/255/14 ms, NSA 2, echo train length 5; ECG-gated partial T2w TSE: TR/TE 2RR/30 ms, NSA 4, echo train length 9; T1w TFE (an MR pulse sequence, which is technically comparable to the T1w magnetization prepared three-dimensional gradient echo MR pulse sequence used by Moody et $\mathrm{al}^{17}$ ): TR/TI/TE 10.3/900/4.0 ms, flip angle 15, NSA 6, inversion prepulse, shot interval time $3000 \mathrm{~ms}$. For the T1w TFE sequence, the FOV was $100 \times 80 \mathrm{~mm}$ with a matrix size of $256 \times 163$ (in-plane resolution $0.39 \times 0.49 \mathrm{~mm}$ ) and a slice thickness of $3.0 \mathrm{~mm}$ and no gap between the slices. For the other sequences, the FOV was $100 \times 80 \mathrm{~mm}$ with a matrix size of $256 \times 205$ (in-plane resolution $0.39 \times 0.39 \mathrm{~mm}$ ) and a slice thickness of $2.5 \mathrm{~mm}$ with a slice gap of $0.5 \mathrm{~mm}$. Nine slices per MR pulse sequence were obtained around the bifurcation. Total investigation time, including patient positioning and image acquisition, was about forty minutes. In case of patient motion, an MR pulse sequence was repeated as long as total imaging time remained around forty minutes.

\section{MRI analysis}

At nine adjoining cross-sectional levels around the carotid bifurcation five MR images (one with each MR pulse sequence) were obtained. The five MR images per cross-sectional level were combined to one MR image set. Two readers (both 4 years experience), blinded for the histological findings and each other results, assessed all MR image sets. All image sets were viewed on a Sun workstation (Sun Ultrasparc, Sun Microsystems, Sunnyvale, CA, USA) using commercially available software (Easyvision, version 4.1, Philips Medical Systems, Best, Netherlands). The MR readers manually traced the vessel wall on a grid and identified regions with homogeneous signal intensity as different regions of interest (ROls). For this purpose, the MR readers used information from all five MR pulse sequences. Together these ROIs covered the complete vessel wall. Subsequently the MR readers classified the relative signal intensity ( $\mathrm{rSI}$ ) of each ROI in each of the five MR weightings of the image set; The SI of the different ROls was compared to that of surrounding muscle tissue closest to the vessel wall, and was classified as "very low" (i.e. almost black), "low" (in between "very low" and "equal"), "equal" (iso-intense to 
surrounding muscle tissue) or "high" (SI higher than surrounding muscle tissue). Each MR reader spent approximately 30-40 minutes per carotid plaque (then all MR weightings were assessed). Cappendijk et al previously found that qualitative and semi-quantitative $M R$ image assessment of the plaque components performed similarly, while semiquantitative analysis was much more time-consuming. ${ }^{16}$ Therefore, we classified the ROls only qualitatively in the present study.

\section{Histology}

After formalin fixation the endarterectomy specimens were divided in 3-mm thick transverse slices, processed and embedded in paraffin. Four$\mu \mathrm{m}$ thick sections of these slices were subjected to hematoxylin-eosin and Elastica von Gieson staining and, were subsequently studied microscopically. In consensus, two investigators (4-10 years experience in vascular histology), who were unaware of the MRI results, identified the major plaque components (fibrous tissue, lipid core, calcification and intraplaque hemorrhage) according to the AHA classification ${ }^{18}$. The boundaries of intra-plaque hemorrhage were often poorly delineated because hemorrhage diffusely infiltrated the lipid core, as also reported by others. ${ }^{15,19}$ Analogous to these authors and Cappendijk et $\mathrm{al}^{16}$, the present study considered the LRNC as the combined areas of lipid core and intraplaque hemorrhage. For quantification purposes, all ROls were traced on color prints made from the histological slices. As with MRI, together these ROls covered the complete vessel wall.

\section{Artery by artery analysis}

An independent investigator matched MRI with histologic slices to ensure that the corresponding carotid artery segments were analyzed. The carotid bifurcation, point of maximum luminal narrowing and crosssectional shape of the vessel wall were used as landmarks in the craniocaudal direction. The carotid bifurcation was defined as the slice showing the carotid bulb. The co-registered MRI and histologic slice at landmark level were used to co-register slices cranially and caudally from the landmark slice. 


\section{Data analysis}

All analyses were performed with SPSS 10.0, Chicago, IL, for Windows 98. The previously published algorithm ${ }^{16}$ categorized four main plaque components in three steps depending on the rSI of the ROI in each of the five MR pulse sequences (Figure 1). Note that in each step a specific combination of MR weightings was used.

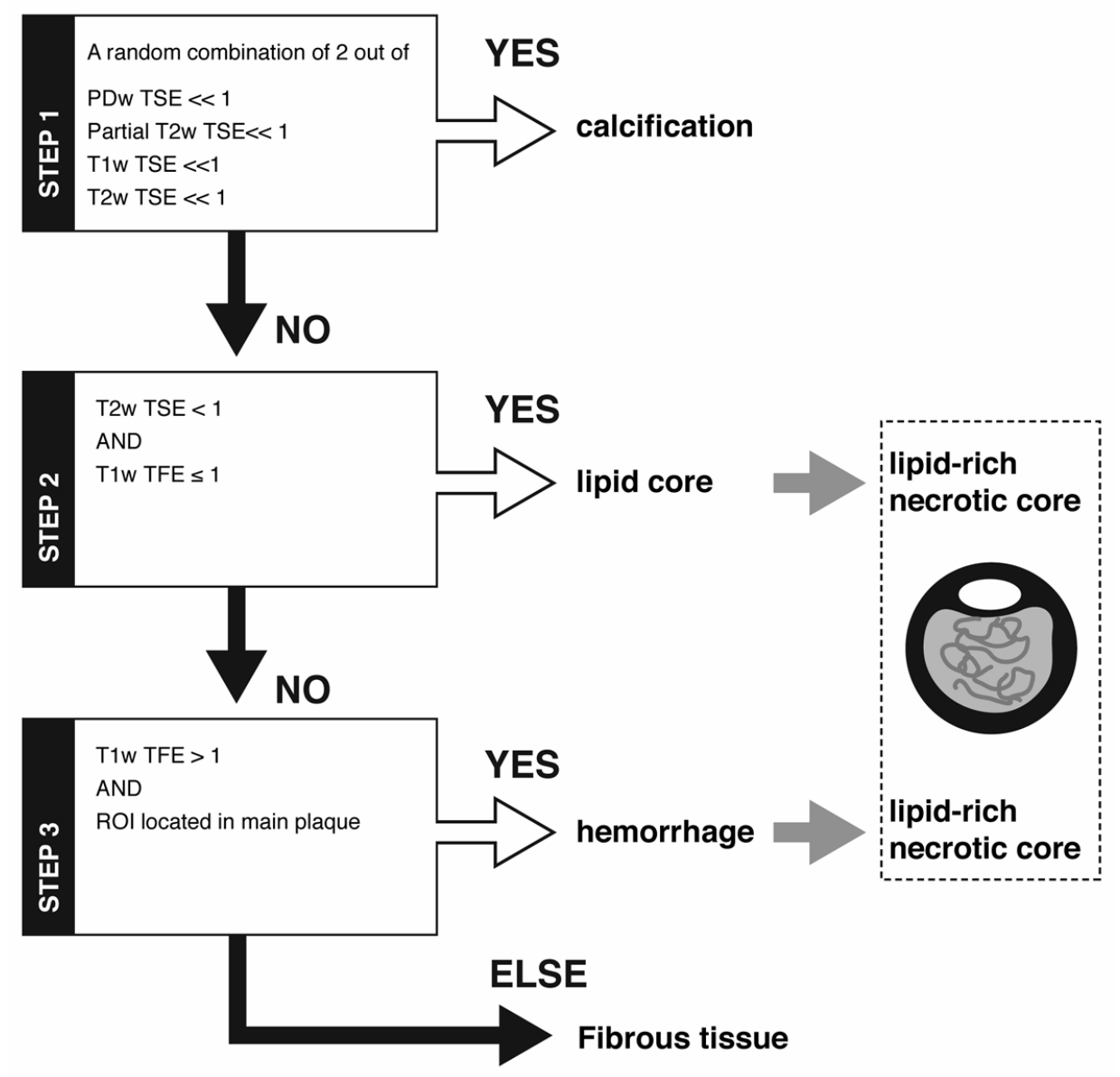

Figure 1. Algorithm for the categorization of plaque components with multisequence MRI. Relative signal intensity (rSI) compared to surrounding muscle tissue; "very low" (i.e. almost black), "low" (in between "very low" and "equal"), "equal" (iso-intense to surrounding muscle tissue) or "high" (SI higher than surrounding muscle tissue). The algorithm can categorize the four plaque components in three steps using the optimal MR pulse sequence combination of five tested MR pulse sequences. ${ }^{10}$ 
The alternative identification of the LRNC based solely on T1w TFE images used "ROI with hyper-intense rSI" AND "ROI confined to main plaque area" as MR criteria.

For both MRI as well as histology, the area of each ROI was expressed as percentage of the total vessel wall area by dividing the number of pixels in each ROI by the total number of pixels comprising the entire vessel wall, and multiplying the results by 100 .

For both MRI and histology, we chose to quantify the amount of the various components (LRNC, calcification, lipid core, intra-plaque hemorrhage and fibrous tissue) with a dedicated scoring system of component scores. The component score is defined as the sum of the area percentages of that particular component in all slices of the complete atherosclerotic plaque. Consequently, the maximum possible value of this score is $9 \times 100=900$, since the maximal craniocaudal size of a plaque was $2.7 \mathrm{~cm}$ (i.e. $=9 \mathrm{MR} /$ histologic slices). This system was introduced because it reflects not only the cross-sectional fraction (area percentage), but also the craniocaudal extent of the LRNC per carotid plaque. For example, a $24 \mathrm{~mm}$ plaque (8 slices in craniocaudal direction) with the same mean LRNC area percentage will have a two-fold larger LRNC score compared to a $12 \mathrm{~mm}$ plaque (4 slices in craniocaudal direction). Given a mean LRNC area percentage of 50 per $3-\mathrm{mm}$ slice the component LRNC score in this example is 400 in the $24-\mathrm{mm}$ plaque vs. 200 in the $12-\mathrm{mm}$ plaque.

\section{Statistical analyis}

Statistical analyses were performed with SPSS 10.0, Chicago, IL, for Windows 98. Interreader and intra-reader agreement of the MR readers for the LRNC was calculated by intraclass correlation coefficients (ICC) with $95 \%$ confidence intervals $(\mathrm{Cl})$. For the intra-reader agreement both MR readers performed a second reading after an interval of more than three months. To avoid bias, image order was scrambled in comparison to the first reading. Agreement between MRI and histology with regard to the amount of LRNC was calculated with linear regression analyses, including a linear regression coefficient and a linear regression equation (i.e. $y=a x+b$, " $y$ " represents histologic score, " $a$ " the slope of the regression line, " $x$ ' the MRI score, and "b" a constant). This method has the advantage that it shows the 
correlation between MRI and histology and also quantifies potential overor underestimation (indicated by $\mathrm{a}$, while b should be close to zero).

Literature showed wide ranges of relative amounts of LRNC within carotid plaques..$^{20,21,22,23}$ Therefore, we estimated the accuracy of MRI for a wide range of LRNC scores (i.e. at histologic component LRNC score-cutoff points of $0,45,90,135,180,315$ and 450). The performance of MRI was quantified by calculating the area under the receiver operating characteristic curve (ROC). Sensitivity and specificity were estimated for each cutoff point using the MRI-LRNC score closest to the upper left corner of the ROC curve as optimal threshold.

Additionally, agreement between multisequence MRI and histology for the various components was represented by linear regression coefficients $(\mathrm{R})$ and determined for both MR readers.

To provide an indication of MRI over- or underestimation of the average relative amounts of plaque components in fifty carotid arteries, the average scores of the plaque components for MRI and histology were compared with a paired sample t-test.

Data are presented as mean \pm standard deviation (s.d.). Data were considered statistically significant if $p<0.05$.

\section{Results}

\section{Subjects}

Out of sixty-four consecutive symptomatic patients data of fifty patients was included. Twelve arteries were excluded because delay between MRI and surgery exceeded 14 days $(n=7)$ or because histology was not available $(n=5)$. Of the 52 arteries left for analysis 2 more were excluded because the MRI was of very poor quality (Figure 2). Of note, only 2 of 64 included patients (3\%) were excluded because of poor image quality. Therefore, analysis could be performed on 50 carotid arteries in 50 patients (age, $67 \pm 8$ years, 37 males). MRI examination was performed $5 \pm 4$ days prior to surgery (range 1-14 days). 


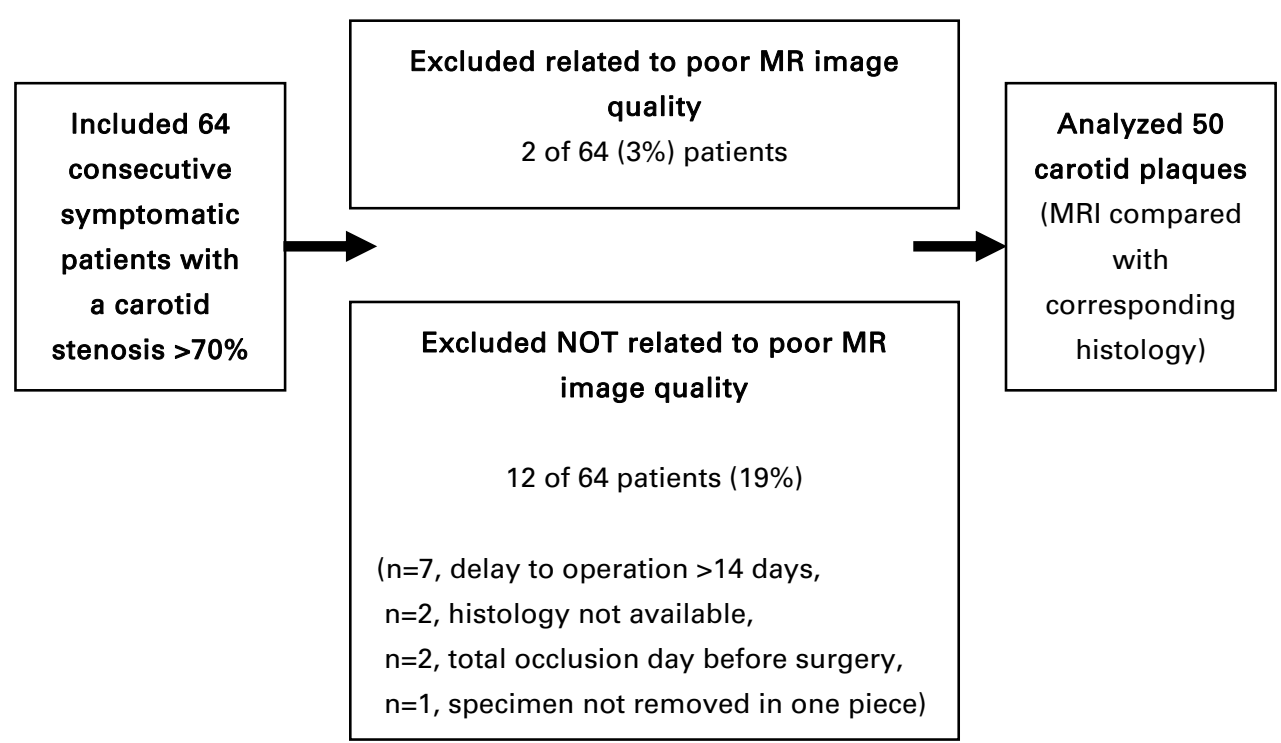

Figure 2. Flow diagram of included and excluded patients. In total 64 patients were included. Fourteen patients were excluded. Only 2 of $64(3 \%)$ were excluded because of poor MR image quality. Analysis was performed on fifty patients.

\section{MRI and histology}

The 50 carotid arteries resulted in 327 MR image sets with 327 corresponding histologic slices, for analysis (6.5 $\pm 1.9 \mathrm{MR}$ image sets per carotid artery; range = 3-9). One hundred-six MR image sets were not analysed because the craniocaudal size of the plaque was smaller than the equivalent of $9 \mathrm{MR}$ slices, i.e. smaller than $2.7 \mathrm{~cm}$; seventeen additional MR image sets were excluded because the corresponding histologic slices were of poor quality. In figure 3 an example is shown of an MR image set as traced with a grid and the corresponding histologic slice. Interreader and intra-reader agreement for quantification of LRNC were excellent and comparable using either multisequence or single sequence T1w TFE images (Table 2). Quantification of the LRNC score with the algorithm as well as solely with T1w TFE images was highly accurate as evidenced by a high correlation between MRI and histology (Table 2, multisequence: $R \geq 0.80, p<0.0001$; single sequence T1w TFE: $R \geq 0.80, p<0.0001)$. Linear regression equations showed that both readers overestimated the size of 
LRNC as determined with MRI with a factor 1.5-1.6 for multisequence MRI, and 1.7 for single sequence T1w TFE MRI compared to histology (Figure 4). Despite this overestimation, both MR readers evidenced a good and similar MRI performance with multisequence and single sequence T1w TFE; areas under the ROC curves were $\geq 0.79$, and sensitivities and specificities $\geq 70 \%$ for all histological cutoff points (Table 1 ).
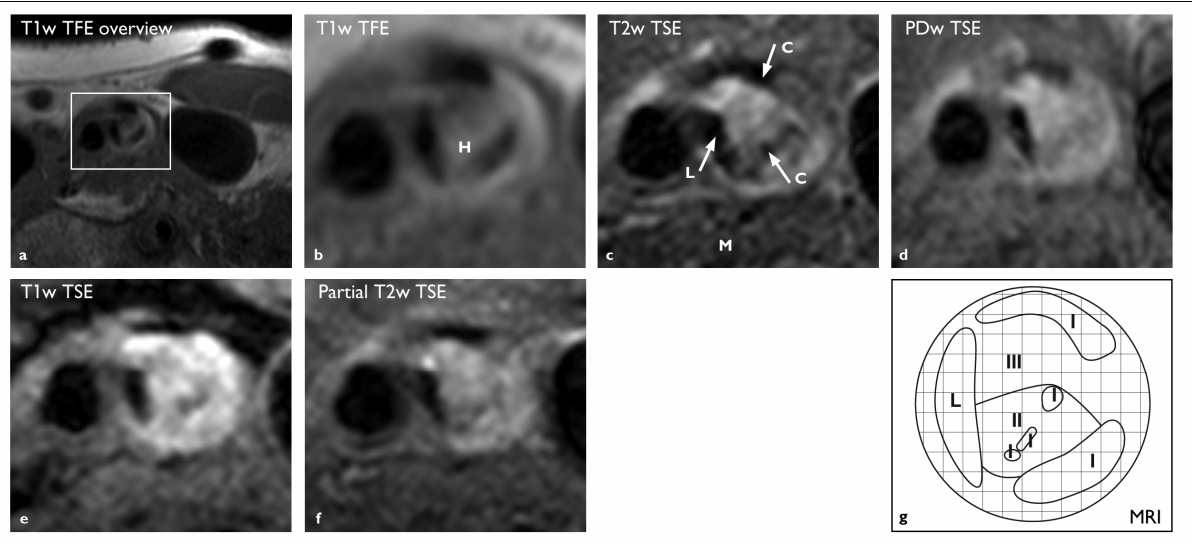

Figure 3 A. Five MR images of the same carotid cross-sectional level, together representing an MR image set. Also shown is the corresponding grid on which one of the MR readers assigned regions of interest (ROIs) using information of the five MR images. MR images are all in transverse plane (a) T1w turbo field echo (TFE) image at carotid bifurcation level, (b) T1w TFE image, (c) Proton Density (PD)w turbo spin echo (TSE) image, (e) T1w TSE image, (f) Partial T2 $w$ TSE image. $H=$ intra-plaque hemorrhage, C (+ arrow)=calcification, $L \quad(+$ arrow)=lumen and $M=$ muscle. $(\mathrm{g})$ grid on which one of the MR readers traced the vessel wall and the ROls with homogeneous relative signal intensity ( $\mathrm{rSI}$ ) based on the information of this particular MR image set. Signal intensity was scored relatively to adjacent muscle tissue (rSI). Percentages of the ROls compared to the whole vessel wall were calculated with the grid (number of pixels of ROI / number of pixels of vessel wall). I=calcification, II=intra-plaque hemorrhage, and III=fibrous tissue.

Figure 3 B. Corresponding histologic slice, see page 87 
Figure 4a. Interreader correlation.

Multisequence MRI

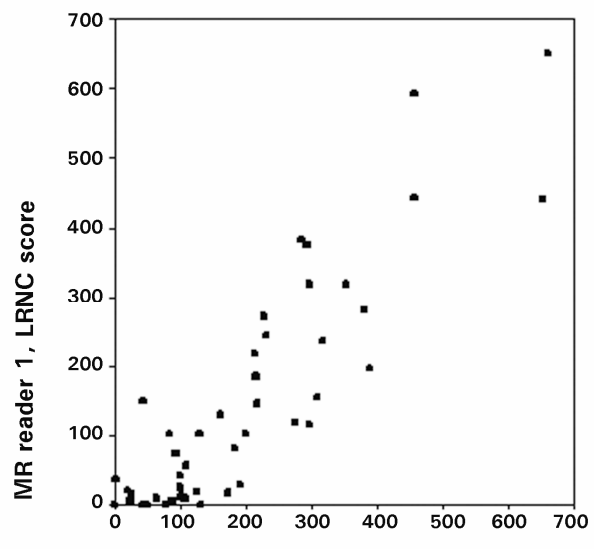

MR reader 2, LRNC score
Single sequence T1 TFE MRI

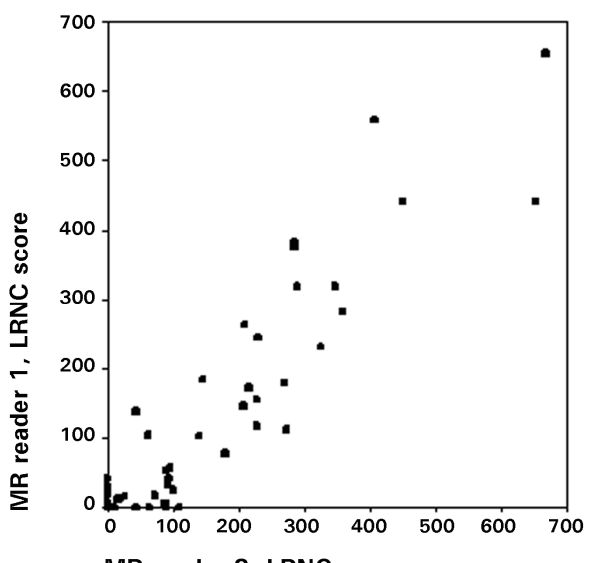

MR reader 2, LRNC score

Intraclass correlation : $0.91(95 \% \mathrm{Cl}, 0.85-0.95)$

Intraclass correlation: $0.86(95 \% \mathrm{Cl}, 0.77-0.94)$

Figure 4b. Correlation between MR reader 1 and histology.

\section{Multisequence MRI}

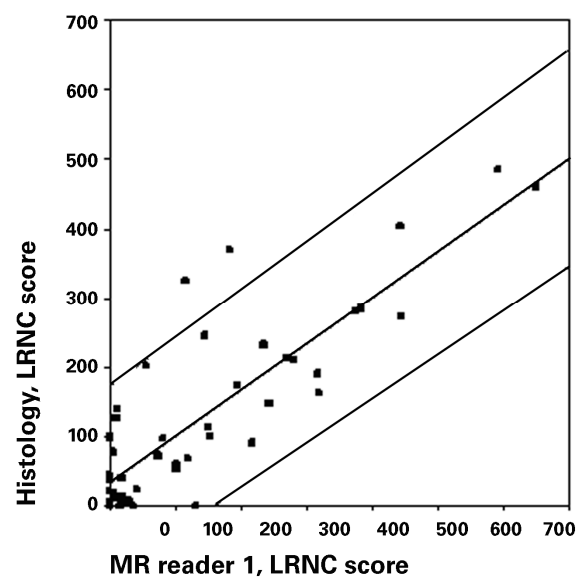

${ }^{*}$ Histology score $=33+0.67 * \mathrm{MRI}$ score

$\mathrm{R}=0.85, \mathrm{p}<.0001$
Single sequence T1 TFE MRI

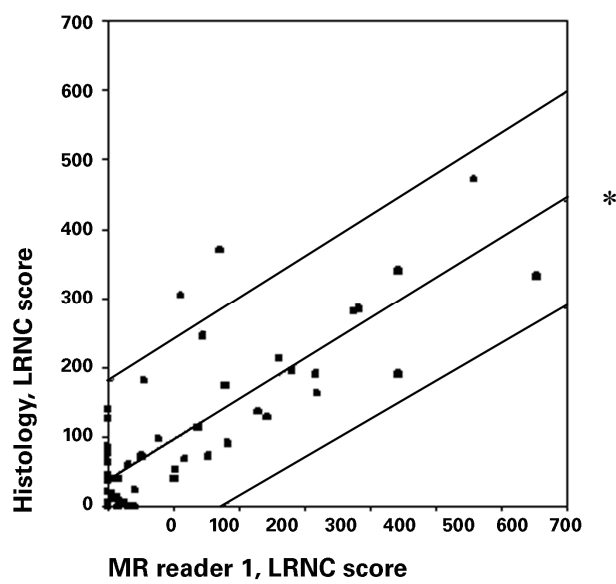

${ }^{*}$ Histology score $=37+0.59 *$ MRI score $\mathrm{R}=0.82, \mathrm{p}<.0001$ 
Figure 4c. Correlation between MR reader 2 and histology.

\section{Multisequence MRI}

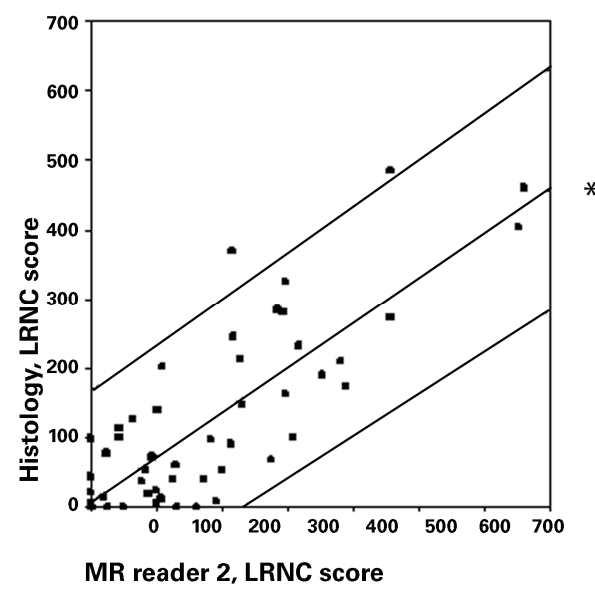

*Histology score $=8+0.64 * \mathrm{MRI}$ score

$\mathrm{R}=0.80, \mathrm{p}<.0001$
Single sequence T1 TFE MRI

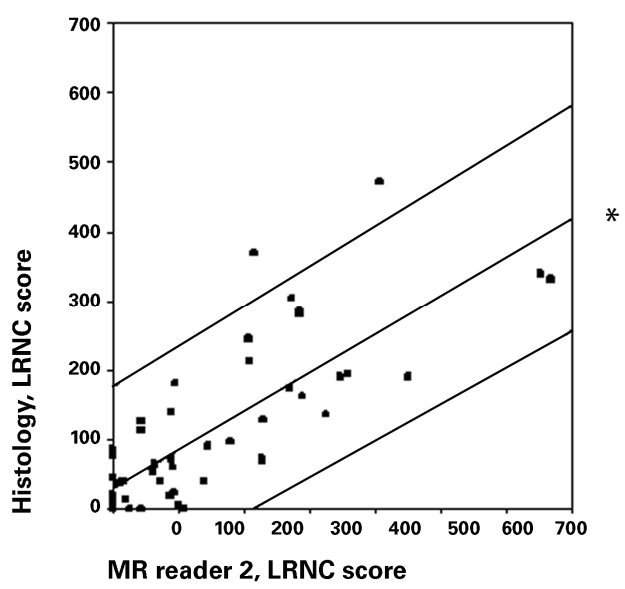

*Histology score $=25+0.58 * \mathrm{MRI}$ score

$\mathrm{R}=0.80, \mathrm{p}<.0001$

Figure 4. Scatter plots of MR interreader agreement and linear regression analysis for the lipid-rich necrotic core (LRNC). LRNC was quantified by the sum of cross-sectional percentages of LRNC per artery $(\mathrm{N}=50)$, resulting in a LRNC score.

$A, B$, and $C$ show scatterplots of multisequence analysis and single sequence T1w TFE analysis; A, Scatter plots and intraclass correlations between MR reader 1 and MR reader 2 (inter-reader agreement) and B, Scatter plots (including reference line and 95\% prediction interval), linear regression equation and linear regression coefficient (R) of MR reader 1 and histology and C, Scatter plots (including reference line and 95\% prediction interval), linear regression equation and linear regression coefficient of MR reader 2 and histology.

Note that with multisequence MRI MR reader 1 and 2 overestimated the LRNC with a factor 1.5-1.6, whereas with the single sequence T1w TFE MRI this overestimation factor was 1.7.

We used the multisequence MR algorithm of Cappendijk et $\mathrm{al}^{16}$, which was not only developed for the identification of LRNC, but also for calcification, lipid core, intra-plaque hemorrhage and fibrous tissue. The linear regression coefficients $(R)$ of both MR readers for the quantification of all these different plaque components separately are listed in Table 2. Intra-plaque hemorrhage was quantified moderate to good by MR reader 1 $(R=0.61, p<0.0001)$ and moderate by $M R$ reader $2(R=0.48, p<0.0001)$. 
High correlation was found for fibrous tissue for both readers $(R \geq 0.74$, $p<0.0001)$. The correlations for calcification were low $(R=0.16(p<0.005)$ and $0.38(p<0.01)$, reader 1 and 2, respectively). Interestingly, no significant correlation was found between MRI and histology for lipid core.

Table 1. Performance of two MR readers to detect different amounts of lipid-rich necrotic core (LRNC). The histologic LRNC treshold quantity is expressed as LRNC score (as explained in the text) and the performance of MRI is estimated by receiver operating characteristic curve $(\mathrm{ROC})$ analysis. $A \cup C=$ area under the curve, sensitivities and specificities (numerator/denominator; $95 \% \mathrm{Cl}$ ). $\mathrm{N}=50$.

I: Results of multisequence MRI of MR readers 1 and 2.

II: Results of single sequence, T1 TFE MRI, of MR readers 1 and 2.

I. MULTISEQUENCE MRI

\begin{tabular}{|c|c|c|c|c|c|c|c|}
\hline \multicolumn{8}{|c|}{ MR reader 1} \\
\hline \multicolumn{8}{|l|}{ Histology } \\
\hline \multirow{2}{*}{$\frac{\text { LRNC score (a.u.)* }}{0}$} & \multirow{2}{*}{$\begin{array}{l}\text { AUC } \\
0.79\end{array}$} & \multirow{2}{*}{$\frac{(95 \% \mathrm{Cl})}{(0.63-0.95)}$} & \multicolumn{2}{|c|}{ Sensitivity } & \multicolumn{3}{|c|}{ / Specificity } \\
\hline & & & $74 \%$ & $(32 / 43 ; 59-86)$ & & $71 \%$ & $(5 / 7 ; 29-96)$ \\
\hline 45 & 0.89 & $(0.79-0.98)$ & $84 \%$ & $(26 / 31 ; 66-95)$ & I & $89 \%$ & $(17 / 19 ; 67-99)$ \\
\hline 90 & 0.85 & $(0.73-0.96)$ & $76 \%$ & $(19 / 25 ; 55-91)$ & & $80 \%$ & $(20 / 25 ; 59-93)$ \\
\hline 135 & 0.93 & $(0.86-1.0)$ & $89 \%$ & $(16 / 18 ; 65-99)$ & I & $84 \%$ & $(27 / 32 ; 67-95)$ \\
\hline 180 & 0.93 & $(0.85-1.0)$ & $86 \%$ & $(12 / 14 ; 57-98)$ & I & $83 \%$ & $(30 / 36 ; 67-95)$ \\
\hline 315 & 0.88 & $(0.73-1.0)$ & $80 \%$ & $(4 / 5 ; 28-99)$ & 1 & $76 \%$ & $(34 / 45 ; 60-87)$ \\
\hline 450 & 1.0 & $(1.0-1.0)$ & $100 \%$ & $(2 / 2 ; 16-100)$ & 1 & $85 \%$ & $(41 / 48 ; 72-94)$ \\
\hline \multicolumn{8}{|l|}{${ }^{*}$ A.u. $=$ arbitrary units } \\
\hline \multicolumn{8}{|c|}{ MR reader 2} \\
\hline \multicolumn{8}{|l|}{ Histology } \\
\hline LRNC score (a.u.)* & AUC & $(95 \% \mathrm{Cl})$ & Sens & ivity & I & Speci & ficity \\
\hline 0 & 0.79 & $(0.64-0.95)$ & $81 \%$ & $(35 / 43 ; 67-92)$ & 1 & $71 \%$ & $(5 / 7 ; 29-96)$ \\
\hline 45 & 0.84 & $(0.74-0.95)$ & $74 \%$ & $(23 / 31 ; 55-88)$ & 1 & $74 \%$ & $(14 / 19 ; 49-91)$ \\
\hline 90 & 0.84 & $(0.72-0.96)$ & $76 \%$ & $(19 / 25 ; 55-91)$ & 1 & $76 \%$ & $(19 / 25 ; 55-91)$ \\
\hline 135 & 0.94 & $(0.87-1.0)$ & $89 \%$ & $(16 / 18 ; 65-99)$ & 1 & $88 \%$ & $(28 / 32 ; 71-96 \%)$ \\
\hline 180 & 0.92 & $(0.84-1.0)$ & $93 \%$ & $(13 / 14 ; 66-100)$ & 1 & $86 \%$ & $(31 / 36 ; 71-95)$ \\
\hline 315 & 0.91 & $(0.80-1.0)$ & $80 \%$ & $(4 / 5 ; 28-99)$ & 1 & $80 \%$ & $(36 / 45 ; 65-90)$ \\
\hline 450 & 0.98 & $(0.93-1.0)$ & $100 \%$ & $(2 / 2 ; 16-100 \%)$ & 1 & $81 \%$ & $(39 / 48 ; 67-91 \%)$ \\
\hline
\end{tabular}

${ }^{*}$ A.u. $=$ arbitrary units 


\begin{tabular}{|c|c|c|c|c|c|c|c|}
\hline & & & reader & & & & \\
\hline LRNC score (a.u.)* & AUC & (95\%Cl) & Sens & vity & 1 & Spec & ficity \\
\hline 0 & 0.79 & $(0.65-0.93)$ & $70 \%$ & $(30 / 43 ; 54-83)$ & 1 & $71 \%$ & $(5 / 7 ; 29-96)$ \\
\hline 45 & 0.83 & $(0.71-0.95)$ & $81 \%$ & $(25 / 31 ; 63-93)$ & l & $84 \%$ & $(16 / 19 ; 60-97)$ \\
\hline 90 & 0.89 & $(0.78-1,0)$ & $86 \%$ & $(18 / 21 ; 64-97)$ & I & $83 \%$ & $(24 / 29 ; 64-94)$ \\
\hline 135 & 0.91 & $(0.82-1.0)$ & $88 \%$ & $(15 / 17 ; 64-99)$ & I & $85 \%$ & $(28 / 33 ; 68-95)$ \\
\hline 180 & 0.93 & $(0.86-1.0)$ & $85 \%$ & $(11 / 13 ; 55-98)$ & l & $84 \%$ & $(31 / 37 ; 68-94)$ \\
\hline 315 & 0.94 & $(0.83-1.0)$ & $100 \%$ & $(4 / 4 ; 40-100)$ & I & $76 \%$ & $(35 / 46 ; 61-87)$ \\
\hline 450 & 0.98 & (0.94-1.0) & $100 \%$ & $(1 / 1 ; 3-100)$ & 1 & $84 \%$ & $(41 / 49 ; 70-93)$ \\
\hline${ }^{*}$ A.u. $=$ arbitrary units & & & & & & & \\
\hline & & & reader & & & & \\
\hline Histology & & & & & & & \\
\hline LRNC score (a.u.)* & AUC & (95\%Cl) & Sens & vity & I & Spec & ficity \\
\hline 0 & 0.81 & $(0.67-0.95)$ & $77 \%$ & $(33 / 43 ; 61-88)$ & / & $71 \%$ & $(5 / 7 ; 29-96)$ \\
\hline 45 & 0.86 & $(0.75-0.96)$ & $77 \%$ & $(24 / 31 ; 59-90)$ & / & $79 \%$ & $(15 / 19 ; 54-94)$ \\
\hline 90 & 0.92 & $(0.85-0.99)$ & $81 \%$ & $(17 / 21 ; 58-95)$ & I & $79 \%$ & $(23 / 29 ; 60-92)$ \\
\hline 135 & 0.95 & $(0.90-1.0)$ & $88 \%$ & $(15 / 17 ; 64-99)$ & I & $88 \%$ & $(29 / 33 ; 72-97)$ \\
\hline 180 & 0.93 & $(0.86-1.0)$ & $85 \%$ & $(11 / 13 ; 55-98)$ & I & $84 \%$ & $(31 / 37 ; 68-94)$ \\
\hline 315 & 0.93 & $(0.81-1.0)$ & $75 \%$ & $(3 / 4 ; 19-99)$ & I & $76 \%$ & $(35 / 46 ; 61-87)$ \\
\hline 450 & 0.94 & $(0.87-1.0)$ & $100 \%$ & $(1 / 1 ; 3-100 \%)$ & I & $82 \%$ & $(40 / 49 ; 68-91 \%)$ \\
\hline
\end{tabular}

${ }^{*}$ A.u.=arbitrary units

The average over- and underestimation of MRI versus histology of the fifty arteries for the component scores of LRNC, calcification, lipid core, intra-plaque hemorrhage and fibrous tissue are presented in Table 3. Based on the average of fifty carotid arteries, MRI significantly overestimated LRNC and intra-plaque hemorrhage and underestimated lipid core and calcification.

In attempt to elucidate the discrepancy between the promising results with the multisequence MRI algorithm for lipid core identification as published by Cappendijk et $\mathrm{al}^{16}$ and the poor correlation between MRI and histology for this component in the current study, two MR readers retrospectively analyzed 15 specific MR image sets of the current study in consensus. MR image sets corresponding to histologic slices with a lipid core comprising $>50 \%$ of the vessel wall area without intra-plaque hemorrhage were selected for this analysis. The lipid cores in this subset were hyper-intense on T1w TFE images in 8 of 15 cases, hyper-intense on 
T2w TSE images in 6 of 15 cases, and iso-intense on T2w images in 5 of 15 cases. Using the algorithm, which identifies lipid core with T1w TFE and T2w TSE images, eight of these lipid cores were categorized incorrectly as intra-plaque hemorrhage; six as fibrous tissue and only one was correctly categorized as lipid core. In contrast, in the study of Cappendijk et $\mathrm{al}^{16} \mathrm{six}$ lipid cores were included and all of these were found to have low rSls on T1w TFE and T2w TSE images.

Table 2. Linear regression coefficients ( $p$-value) of multisequence, and single sequence (T1w TFE) MRI and histology for the lipid-rich necrotic core (LRNC) and the separate plaque components. Linear regression coefficients were calculated based on the tissue scores per artery (total fifty arteries) for MR reader 1 and MR reader 2 .

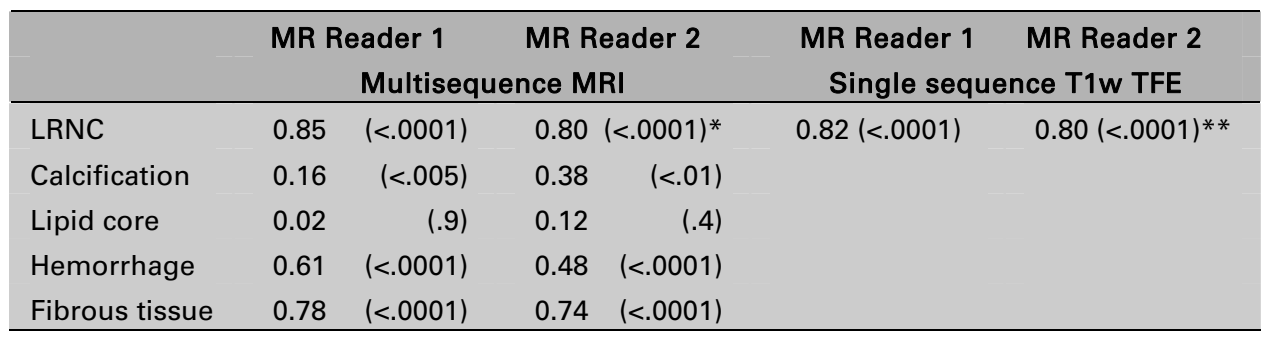

* Interreader intraclass correlation (ICC, 95\% Cl): 0.86 (0.77-0.94)

Intra-reader ICC, MR reader 1: 0.94 (0.89-0.99)

Intra-reader ICC, MR reader 2: 0.75 (0.43-1.0)

* Interreader ICC: 0.91 (0.85-0.95)

Intra-reader ICC, MR reader 1: 0.94 (0.87-0.97)

Intra-reader ICC, MR reader 2: $0.75(0.51-0.88)$

Table 3. Average component scores (standard deviation, range, p-value) based on multisequence MRI of fifty carotid arteries for lipid-rich necrotic core (LRNC), calcification, lipid core, intra-plaque hemorrhage (IPH) and fibrous tissue, and based on single sequence T1w TFE MRI for LRNC.

\begin{tabular}{lcrlrc}
\hline & \multicolumn{2}{l}{ Histology } & \multicolumn{2}{l}{ MRI mulisequence } & \multicolumn{1}{c}{$\begin{array}{c}\text { Single sequence } \\
\text { T1w TFE }\end{array}$} \\
\hline LRNC & 112 & $(116,0-471)$ & 157 & $(157,0-656,<.005)$ & $139(160,0-661,<.05)$ \\
Calcification & 39 & $(57,0-294)$ & 14 & $(16,0-72,<.005)$ & - \\
Lipid core & 65 & $(96,0-370)$ & 18 & $(28,0-131,<.005)$ & - \\
IPH & 47 & $(66,0-279)$ & 139 & $(159,0-652,<.0001)$ & - \\
Fibrous tissue & 502 & $(149,173-861)$ & 483 & $(150,125-851,=.20)$ & - \\
\hline
\end{tabular}




\section{Discussion}

The presence and size of lipid-rich necrotic core (LRNC) in atherosclerotic plaque are regarded as an important indicators of plaque vulnerability. ${ }^{9,10,11}$ However, little is known about the ability of MRI to quantify the amount of LRNC present in plaques. In the present study, we quantified the amount of LRNC in carotid plaques at multiple crosssectional levels with a dedicated scoring system. We found both multisequence as well as single sequence T1w TFE MRI capable of accurate and reproducible quantification of the amount of LRNC.

Although it is believed that larger LRNCs are associated with higher risk of stroke, the relation between size of the LRNC and its development over time and relations to symptoms and therapy are unknown at present. Various publications have described a wide range of amounts of LRNC in endarterectomy specimens of patients who suffered a recent TIA or stroke. ${ }^{20,21,22,23}$ It is likely that identification alone will not be enough for risk assessment and therefore, accurate quantification of a wide range of amounts of LRNC is an important prerequisite before implementation of MRI plaque imaging as a clinical tool. This hypothesis is supported by the recent study of Takaya et $\mathrm{al}^{24}$ who found that an increase of LRNC area as detected with multisequence MRI was a significant predictor of future ipsilateral cerebrovascular events. In the current study we applied Cappendijk's previously published algorithm ${ }^{16}$, and found that the quantification of the amount of LRNC with MRI correlated very well with histology. In addition, we found that these results were highly reproducible among two independent MR readers. The more salient finding of this study, however, is that quantification of LRNC based on single sequence T1w TFE images resulted in similar high correlations, sensitivities and specificities. This finding is very important because the T1w TFE acquisition takes less than four minutes, which makes single sequence T1w TFE quantification of the LRNC superior to the multisequence algorithm due to the much shorter imaging and interpretation times. Both methods have potential for identification and quantification of the LRNC in populations with a wide range of sizes of carotid LRNC and will enable follow up of the LRNC size in carotid plaques over time in relation to symptoms and therapy. However, analysis of just 
one MR pulse sequence will be much easier compared to acquisition of five sequences and will therefore be more easily applicable in daily clinical practice.

In the present study the multisequence MRI algorithm had difficulties to differentiate lipid core from intra-plaque hemorrhage. The most likely reason for this finding, as corroborated in a recent study by Saam et $\mathrm{al}^{15}$, is that it is often hard to demarcate intra-plaque hemorrhage from lipid core due to the diffuse distribution of intra-plaque hemorrhage in the lipid core. It is obvious that when histologic differentiation between lipid core and intra-plaque hemorrhage is difficult, despite the much higher in-plane resolution of microscopy compared to MRI, that differentiation with MRI will be virtually impossible. Therefore, instead of focussing on detection of these components separately, we aimed to identify the lipid-rich necrotic core, containing both lipid core and intra-plaque hemorrhage.

In a retrospective analysis of large histologic proven lipid cores (without intra-plaque hemorrhage) as found in the current study, we demonstrated that the algorithm ${ }^{16}$ incorrectly classified lipid core as intraplaque hemorrhage in the majority of cases (8 of 15). This is a second explanation for the poor correlation between MRI and histology for quantification of the amount of lipid core and moderate correlation for the quantification of the amount of intra-plaque hemorrhage in the current study, while the overall ability to detect and quantify the LRNC score (lipid core and intra-plaque hemorrhage) was good.

We found high correlations between multisequence MRI and histology for the quantification of fibrous tissue. For calcification on the other hand we found little correlation between MRI and histology. Calcifications are usually distributed unevenly and in small quantities, which can be obscured in MR images because of partial volume effects. The additional use of a time of flight (TOF) "bright blood" MR pulse sequence may improve the detection of calcification, because it amplifies contrast between high intensity lumen and low intensity calcifications adjacent to the lumen. Since the scores of calcification in plaque were small in the current study, underestimation of calcification will have hardly hampered quantification of the LRNC score.

In the present study, we chose to quantify the amount of LRNC in the plaques with a dedicated scoring system (tissue score per carotid plaque). 
This component score, compared to using the average amount per plaque or slice, also reflects the extent of the LRNC in the craniocaudal direction. Future studies are warranted to test the hypothesis that a larger LRNC score corresponds to a higher risk of thrombo-embolic complications.

Limitations and future developments

Although the assessment of area percentages of LRNC at multiple cross-sectional levels was laborious, the technique was found to be reliable as demonstrated by high inter- and intra-reader agreement and high correlation between MRI and histology. Even though our LRNC score has no unit, it does provide a reliable means to assess the amount of LRNC present in a carotid plaque. In future studies we would prefer to analyse volumes $\left(\mathrm{mm}^{3}\right)$, but, in the present study we lacked the means to do so.

In the present study, partial volume effects in MR images and nonuniform shrinkage (of unknown extent) of the histologic specimens may have resulted in over- or underestimation of plaque components. However, partial volume and shrinkage are expected to be random phenomena and therefore we presume that their effects will be on average constant in time. Therefore, in studies, where plaques are serially assessed over time, this may not be an issue.

Recently, computerized plaque component quantification with supervised classifiers showed some promising initial results ${ }^{25,26}$ but in our experience $^{26}$ it is currently still much more time-consuming compared to the present method.

The present study demonstrated the feasibility of accurate quantification of the amount of LRNC by non-contrast-enhanced MRI. These results might be further improved adding contrast-enhanced sequences, since it was previously described that contrast-enhanced imaging might help to discriminate fibrous cap from lipid $\operatorname{cor}^{27,28}$ and, moreover, that it enables quantification of neovascularization. ${ }^{29}$

\section{Conclusions}

The present study prospectively showed that both multisequence and single sequence T1w TFE MRI provide accurate, reproducible, and fast relative measures to quantify the amount of LRNC in carotid plaque. This method may be promising to follow atherosclerotic plaques over time and 
assess size of the LRNC in relation to thrombo-embolic events, effects of statins on plaques and/or the relation to other risk factors.

\section{Acknowledgements}

This work was financially supported by a grant from the Dutch Heart Foundation (2000.173).

The authors would like to acknowledge Marc Geerlings, Department of Radiology, Maastricht University Hospital, Maastricht, The Netherlands, for his extensive informatics and computer support and database management.

Dr. Leiner is an NOW-VENI fellow (grant number 916.46.034). 


\section{References}

1. Falk E, Shah PK, Fuster V. Coronary plaque disruption. Circulation. 1995;92:657-671.

2. Virmani R, Kolodgie FD, Burke AP, Farb A, Schwartz SM. Lessons from sudden coronary death: a comprehensive morphological classification scheme for atherosclerotic lesions. Arterioscler Thromb Vasc Biol. 2000;20:1262-1275.

3. Beneficial effect of carotid endarterectomy in symptomatic patients with high-grade carotid stenosis. North American Symptomatic Carotid Endarterectomy Trial Collaborators. N Engl J Med. 1991;325:445-453.

4. MRC European Carotid Surgery Trial: interim results for symptomatic patients with severe (70-99\%) or with mild (0-29\%) carotid stenosis. European Carotid Surgery Trialists' Collaborative Group. Lancet. 1991;337:1235-1243.

5. Randomised trial of endarterectomy for recently symptomatic carotid stenosis: final results of the MRC European Carotid Surgery Trial (ECST). Lancet. 1998;351:1379-1387.

6. Barnett HJ, Taylor DW, Eliasziw M, Fox AJ, Ferguson GG, Haynes RB, Rankin RN, Clagett GP, Hachinski VC, Sackett DL, Thorpe KE, Meldrum $H E$, Spence JD. Benefit of carotid endarterectomy in patients with symptomatic moderate or severe stenosis. North American Symptomatic Carotid Endarterectomy Trial Collaborators. N Engl J Med. 1998;339:1415-1425.

7. Rothwell PM, Eliasziw M, Gutnikov SA, Fox AJ, Taylor DW, Mayberg $\mathrm{MR}$, Warlow CP, Barnett HJ. Analysis of pooled data from the randomised controlled trials of endarterectomy for symptomatic carotid stenosis. Lancet. 2003;361:107-116.

8. Rothwell PM, Goldstein LB. Carotid endarterectomy for asymptomatic carotid stenosis: asymptomatic carotid surgery trial. Stroke. 2004;35:2425-2427.

9. Gutstein DE, Fuster, V. Pathophysiology and clinical significance of atherosclerotic plaque rupture. Cardiovasc Res. 1999;41:323-333. 
10. Naghavi M, Libby P, Falk E, Casscells SW, Litovsky S, Rumberger J, Badimon JJ, Stefanadis C, Moreno P, Pasterkamp G, Fayad Z, Stone PH, Waxman S, Raggi $P$, Madjid M, Zarrabi A, Burke A, Yuan C, Fitzgerald PJ, Siscovick DS, de Korte CL, Aikawa M, Juhani Airaksinen KE, Assmann G, Becker CR, Chesebro JH, Farb A, Galis ZS, Jackson C, Jang IK, Koenig W, Lodder RA, March K, Demirovic J, Navab M, Priori SG, Rekhter MD, Bahr R, Grundy SM, Mehran R, Colombo A, Boerwinkle E, Ballantyne C, Insull W, Jr., Schwartz RS, Vogel R, Serruys PW, Hansson GK, Faxon DP, Kaul S, Drexler H, Greenland P, Muller JE, Virmani R, Ridker PM, Zipes DP, Shah PK, Willerson JT. From vulnerable plaque to vulnerable patient: a call for new definitions and risk assessment strategies: Part I. Circulation. 2003;108:1664-1672.

11. Falk E. Stable versus unstable atherosclerosis: Clinical aspects. Am Heart J. 1999;138:S421-S425.

12. Kolodgie FD, Gold HK, Burke AP, Fowler DR, Kruth HS, Weber DK, Farb A, Guerrero LJ, Hayase M, Kutys R, Narula J, Finn AV, Virmani R. Intraplaque hemorrhage and progression of coronary atheroma. $\mathrm{N}$ Engl J Med. 2003;349:2316-2325.

13. Fayad ZA, Fuster V. Clinical imaging of the high-risk or vulnerable atherosclerotic plaque. Circ Res. 2001;89:305-316.

14. Yuan C, Mitsumori LM, Beach KW, Maravilla KR. Carotid atherosclerotic plaque: noninvasive $M R$ characterization and identification of vulnerable lesions. Radiology. 2001;221:285-299.

15. Saam T, Ferguson MS, Yarnykh VL, Takaya N, Xu D, Polissar NL, Hatsukami TS, Yuan C. Quantitative evaluation of carotid plaque composition by in vivo MRI. Arterioscler Thromb Vasc Biol. 2005;25:234-239.

16. Cappendijk VC, Cleutjens KB, Kessels AG, Heeneman S, Schurink GW, Welten RJ, Mess WH, Daemen MJ, van Engelshoven JM, Kooi ME. Assessment of human atherosclerotic carotid plaque components with multisequence MR imaging: initial experience. Radiology. 2005;234:487492. 
17. Moody AR, Murphy RE, Morgan PS, Martel AL, Delay GS, Allder S, MacSweeney ST, Tennant WG, Gladman J, Lowe J, Hunt BJ. Characterization of complicated carotid plaque with magnetic resonance direct thrombus imaging in patients with cerebral ischemia. Circulation. 2003;107:3047-3052.

18. Stary HC, Chandler AB, Dinsmore RE, Fuster V, Glagov S, Insull W, Jr., Rosenfeld ME, Schwartz CJ, Wagner WD, Wissler RW. A definition of advanced types of atherosclerotic lesions and a histological classification of atherosclerosis. A report from the Committee on Vascular Lesions of the Council on Arteriosclerosis, American Heart Association. Arterioscler Thromb Vasc Biol. 1995;15:1512-1531.

19. Yuan C, Mitsumori LM, Ferguson MS, Polissar NL, Echelard D, Ortiz G, Small R, Davies JW, Kerwin WS, Hatsukami TS. In vivo accuracy of multispectral magnetic resonance imaging for identifying lipid-rich necrotic cores and intraplaque hemorrhage in advanced human carotid plaques. Circulation. 2001;104:2051-2056.

20. Montauban van Swijndregt $A D$, Elbers $H R$, Moll FL, de Letter J, Ackerstaff RG. Cerebral ischemic disease and morphometric analyses of carotid plaques. Ann Vasc Surg. 1999;13:468-474.

21. Tegos TJ, Sohail M, Sabetai MM, Robless P, Akbar N, Pare G, Stansby G, Nicolaides AN. Echomorphologic and histopathologic characteristics of unstable carotid plaques. Am J Neuroradiol. 2000;21:1937-1944.

22. Seeger JM, Barratt E, Lawson GA, Klingman N. The relationship between carotid plaque composition, plaque morphology, and neurologic symptoms. J Surg Res. 1995;58:330-336.

23. Arapoglou B, Kondi-Pafiti A, Katsenis K, Dimakakos P. The clinical significance of carotid plaque haemorrhage. Int Angiol. 1994;13:323326.

24. Takaya N, Yuan C, Chu B, Saam T, Underhill H, Cai J, Tran N, Polissar NL, Isaac C, Ferguson MS, Garden GA, Cramer SC, Maravilla KR, Hashimoto B, Hatsukami TS. Association Between Carotid Plaque Characteristics and Subsequent Ischemic Cerebrovascular Events. A Prospective Assessment With MRI--Initial Results. Stroke. 2006;37:818823. 
25. Liu F, Xu D, Ferguson MS, Chu B, Saam T, Takaya N, Hatsukami TS, Yuan C, Kerwin WS. Automated in vivo segmentation of carotid plaque MRI with Morphology-Enhanced probability maps. Magn Reson Med. 2006;55:659-668.

26. Hofman JM, Branderhorst WJ, ten Eikelder HM, Cappendijk VC, Heeneman S, Kooi ME, Hilbers PA, ter Haar Romeny BM. Quantification of atherosclerotic plaque components using in vivo MRI and supervised classifiers. Magn Reson Med. 2006;55:790-799.

27. Wasserman BA, Smith WI, Trout HH, 3rd, Cannon RO, 3rd, Balaban RS, Arai AE. Carotid artery atherosclerosis: in vivo morphologic characterization with gadolinium-enhanced double-oblique MR imaging initial results. Radiology. 2002;223:566-573.

28. Cai J, Hatsukami TS, Ferguson MS, Kerwin WS, Saam T, Chu B, Takaya $\mathrm{N}$, Polissar NL, Yuan C. In vivo quantitative measurement of intact fibrous cap and lipid-rich necrotic core size in atherosclerotic carotid plaque: comparison of high-resolution, contrast-enhanced magnetic resonance imaging and histology. Circulation. 2005;112:3437-3444.

29. Kerwin W, Hooker A, Spilker M, Vicini P, Ferguson M, Hatsukami T, Yuan C. Quantitative magnetic resonance imaging analysis of neovasculature volume in carotid atherosclerotic plaque. Circulation. 2003;107:851-856. 


\section{Chapter \\ 5}

\section{Magnetic resonance imaging quantification of lipid-rich necrotic core size in carotid atherosclerotic plaque of symptomatic and asymptomatic patients}

VC Cappendijk, AGH Kessels, S Heeneman, KBJM Cleutjens, GWH Schurink, RJThJ Welten, WH Mess, RJ van Suylen, T Leiner, MJAP Daemen, JMA van Engelshoven, ME Kooi

Submitted to European Radiology

This work was supported by a grant from the Dutch Heart Foundation (2000.173) 


\section{Abstract}

Purpose: Pathological studies established that a large lipid-rich necrotic core (LRNC) is an important feature of vulnerable atherosclerotic plaque. The purpose of the present study was to investigate potential differences in size of the LRNC in carotid plaques of symptomatic patients versus asymptomatic patients with high-grade carotid stenosis.

Materials and Methods: Thirty-seven patients with carotid stenosis $>70 \%$ with $(n=26)$ or without $(n=11)$ symptoms were included. Three independent MR readers quantified the amount of LRNC with a previously validated $T 1$ weighed $(w)$ turbo field echo (TFE) pulse sequence. The relative amount of LRNC was defined as sum of cross-sectional area percentages LRNC per carotid plaque.

Results: The mean LRNC score was $116 \pm 129$ and $59 \pm 62$ for symptomatic and asymptomatic patients, respectively $(p<0.05)$. Interreader agreement for the three MR readers was high with intraclass correlation coefficient (ICC, $95 \% \mathrm{Cl}$ ) of $0.75(0.62-0.85)$. Symptomatic patients had a wide range in LRNC scores (0-424), while the range was much lower in the asymptomatic group (0-170).

Conclusions: On average, symptomatic patients have a significantly larger LRNC compared to asymptomatic patients as quantified with T1w TFE images. Future studies are warranted to investigate whether a large LRNC as quantified with MRI can be used to identify carotid plaques with increased risk of stroke. 


\section{Introduction}

It is well known that patients with severe carotid stenosis and recent symptoms have a high risk of (recurrent) stroke. Two major randomized trials and additional analyses of the results of these studies ${ }^{1,2,3,4,5}$ found an overall significantly decreased risk of stroke in operated (endarterectomy) patients with symptomatic high grade carotid stenosis (70-99\%). In asymptomatic patients (defined as the absence of stroke or transient ischemic attack (TIA) for at least 6 months) with significant carotid stenosis the benefits of surgery in terms of reduced risk of disabling or fatal stroke are much smaller. ${ }^{6,78}$ However, even in the group of symptomatic patients with high-grade carotid stenosis only about twenty percent will suffer a major stroke on medical treatment alone within three years after the first symptoms, ${ }^{9}$ which means that $80 \%$ may be exposed to unnecessary surgical intervention with substantial associated morbidity and mortality (surgical risk of stroke and/or death is $7.1 \%)^{5}$.

In light of the above it is imperative to identify additional risk factors for stroke to come to a better selection of patients that may benefit from surgery. The underlying pathology of patients with carotid stenosis is the presence of an atherosclerotic plaque in the carotid artery. Atherosclerotic plaque formation starts with the proces of outward remodeling and progresses to arterial stenosis only in later phases of disease. Luminography as used in clinical practice will therefore underestimate the true extent of disease. Furthermore, it is unable to provide information about plaque composition.

Ischemic events are mainly caused by rupture of vulnerable atherosclerotic plaque with subsequent thrombus formation. ${ }^{10,11}$ Several pathological studies have found that a large lipid-rich necrotic core (LRNC) is an important feature of vulnerable plaque..$^{12,13,14}$

Previously, it has been shown by us (unpublished data) and others ${ }^{15}$ that non-invasive magnetic resonance imaging (MRI) can quantify the LRNC in vivo in carotid plaque.

Recently, we (unpublished data) found high correlations between MRI and histology for quantification of the relative amount of LRNC (linear regression coefficient of 0.81 and $0.80, p<0.0001$, respectively for two independent MR readers) using a fast high-resolution T1 weighted (w) 
turbo field echo (TFE) MR pulse sequence. This study also found (unpublished data) high sensitivities and specificities for a wide range of relative amounts of LRNC were high (sensitivities: $70 \%-100 \%$ and specificities: $71-89 \%)$.

The aim of the present study was to investigate potential differences in the average amount of LRNC in carotid plaques of symptomatic patients with high-grade stenosis, as quantified with T1w TFE images, compared to asymptomatic patients with high-grade carotid stenosis.

\section{Materials and Methods}

\section{Patient selection}

The institutional medical ethics committee approved the study and all subjects gave written informed consent prior to inclusion. Both symptomatic and asymptomatic patients with a carotid stenosis of $>70 \%$ (symptomatic or asymptomatic) as diagnosed by duplex ultrasonography were referred for inclusion by vascular surgeons ( $>10$ years experience).

Symptomatic patients were referred to the vascular surgeon because they had suffered TIA or stroke of the ipsilateral carotid artery. Asymptomatic patients were follow-up patients of the vascular surgeon because they suffered from peripheral arterial occlusive disease or they had undergone previous contralateral carotid endarterectomy. The definition of symptomatic and asymptomatic carotid patients was similar to the NASCET (North American Symptomatic Carotid Surgery Trial), ECST (European Carotid Surgery Trial) and ACST (Asymptomatic Carotid Surgery Trial): ${ }^{5,7}$ These trials considered patients to be symptomatic when they had suffered stroke or TIA within 4-6 months prior to inclusion and asymptomatic when they had not suffered stroke or TIA for at least 6 months before inclusion. In the current study, we considered patients to be symptomatic when they had suffered one or more ipsilateral TIA or strokes within three months before inclusion. Whether or not a patient was considered symptomatic was based on patient history and clinical examination. We considered patients to be asymptomatic when they had not suffered any TIA or stroke for at least six months on the date of 
inclusion. There was an 18 month follow up period after MRI investigation for all patients.

\section{MRI protocol}

All MRI examinations were done on a 1.5-T whole body MRI scanner (Philips Intera, release 8.1.2, Philips Medical Systems, Best, The Netherlands) using a commercially available radio frequency surface coil with a diameter of $47 \mathrm{~mm}$ (Philips Medical Systems).

Survey scans were used to determine the level of the carotid bifurcation and to verify correct position of the surface coil. When the coil was in the correct position a T1-weighted (w) turbo field echo (TFE) MR pulse sequence was acquired with the following parameters: TR/TI/TE $10.3 / 900 / 4.0 \mathrm{~ms}$, flip angle $15^{\circ}$, NSA 6 , inversion prepulse, shot interval time $3000 \mathrm{~ms}$. Nine slices were obtained around the bifurcation with a slice thickness of $3 \mathrm{~mm}$. The field of view was $100 \times 80 \mathrm{~mm}$ and matrix size was $256 \times 163$, yielding an in-plane resolution of $0.39 \times 0.49 \mathrm{~mm}^{2}$. Nominal scan time was 3 minutes 38 seconds. Total investigation time was less than 15 minutes.

\section{Image evaluation}

Three independent MR readers, unaware of patients histories and medical information, individually assessed all carotid plaques. For the intra-reader agreement all three MR readers performed a second reading after an interval of more than three months. Only slices containing plaque, plus one slice situated caudally and one slice situated cranially of the plaque (maximum is 9 slices), were evaluated on a Sun workstation (Sun Ultrasparc, Sun Microsystems, Sunnyvale, CA, USA) with commercially available post-processing software (Philips Easyvision, version 4.1, Philips Medical Systems).

For each atherosclerotic plaque, readers determined the relative amount of LRNC (i.e. lipid core including possible intra-plaque hemorrhage) in a similar fashion as in a previous study (unpublished data). In brief, MR readers traced the vessel wall and manually marked regions of interest (ROI) with hyperintense signal intensity relative to surrounding muscle tissue. Readers also assessed whether the ROI was located within the main plaque area (in contrast to a mildly thickened 
atherosclerotic vessel wall). Subsequently, the area of each hyperintense $\mathrm{ROI}$ was expressed as percentage of the total vessel wall area by assessing the number of pixels of the ROI. An ROI was considered to be LRNC if it was hyperintense relative to surrounding muscle tissue, and was located within the main plaque area. The sum of the cross-sectional area percentages of LRNC determined the relative amount of LRNC per artery (i.e. LRNC score). Since a maximum of nine MR slices were assessed the maximal possible LRNC score was $9 \times 100=900$.

\section{Statistical analysis}

Data analysis was performed using SPSS 10.0 (SPSS, Inc., Chicago, IL, USA) for Windows 98. Differences in patient characteristics between the symptomatic and asymptomatic groups (stroke risk factors, aspirin, and statin use) were investigated with a Chi-squared test, except for a possible difference in age, which was tested with a t-test. Data were considerd statistically significant if $\mathrm{p}<0.05$.

Intra-reader and inter-reader agreement of the three MR readers for quantification of the LRNC score were expressed as intraclass correlation coefficients (ICC, 95\% Cl). A t-test was used to determine if there was a difference in LRNC score between groups of symptomatic and asymptomatic patients. Differences were tested one-tailed based on the assumption that plaques of symptomatic patients have a higher LRNC score. In addition, we tested the global hypothesis that the results were equal for all three MR readers using the ordinary least square test. ${ }^{16,17}$

\section{Results}

Thirty-seven consecutive patients (age $66 \pm 8$ years, 30 males) with a high grade carotid stenosis were included. Twenty-six of these patients were symptomatic and eleven asymptomatic. There were no significant differences in patient characteristics between the symptomatic and asymptomatic patients (Table 1 ).

A mean of $6.7 \pm 1.5 \mathrm{MR}$ slices were assessed per carotid artery. MR image assessment took approximately 15 minutes per patient. Intra-reader and interreader agreement were high with an intra-reader ICC of 0.75 
(0.63-0.83) and interreader ICC of $0.72(0.57-0.83)$. All MR readers found larger LRNC in the plaques of symptomatic patients. Differences in mean LRNC score between the plaques of the group of symptomatic and asymptomatic patients are listed in table 2. The distribution of LRNC scores in both groups is shown in Figure 1. Symptomatic patients exhibited a wide range of LRNC scores (0-424), whereas the range in scores was much lower in the asymptomatic group (0-170). It is important to note that a considerable number of symptomatic patients also had a small LRNC.

Table 1. Patient characteristics.

\begin{tabular}{|c|c|c|c|}
\hline Risk factor & $\begin{array}{c}\text { Symptomatic } \\
(n=26)\end{array}$ & $\begin{array}{c}\text { Asymptomatic } \\
(n=11)\end{array}$ & $\begin{array}{c}\text { Statistical significance } \\
\mathrm{p} \text {-value }\end{array}$ \\
\hline Male & $20 \quad(77 \%)$ & $10 \quad(91 \%)$ & 0.32 \\
\hline Age (years) & $66 \pm 8$ & $63 \pm 8$ & 0.29 \\
\hline Diabetes & $4 \quad(15 \%)$ & $3 \quad(27 \%)$ & 0.40 \\
\hline Smoking & $15 \quad(58 \%)$ & $7 \quad(64 \%)$ & 0.74 \\
\hline Hypertension & $14 \quad(54 \%)$ & $7 \quad(64 \%)$ & 0.58 \\
\hline Hypercholesterolemia & $7 \quad(27 \%)$ & $6 \quad(55 \%)$ & 0.11 \\
\hline CVD* & $9 \quad(35 \%)$ & $7 \quad(64 \%)$ & 0.10 \\
\hline Aspirin & $26 \quad(100 \%)$ & $11(100 \%)$ & 1.0 \\
\hline Statins & $11 \quad(42 \%)$ & $5 \quad(46 \%)$ & 0.86 \\
\hline
\end{tabular}

${ }^{*}$ CVD = cardio-vascular disease other than carotid stenosis, i.e. coronary artery disease and peripheral artery disease.

Table 2. Mean lipid-rich necrotic core (LRNC) scores \pm standard deviation (range) in the symptomatic $(n=26)$ and asymptomatic $(n=11)$ groups of patients

\begin{tabular}{lcccc}
\hline & \multicolumn{2}{c}{ LRNC score } & & \\
& Symptomatic & Asymptomatic & Difference & p-value* \\
\hline MR reader 1 & $132 \pm 119(0-438)$ & $74 \pm 78(0-208)$ & 58 & 0.07 \\
MR reader 2 & $84 \pm 119(0-519)$ & $51 \pm 63(0-176)$ & 33 & 0.20 \\
MR reader 3 & $132 \pm 172(0-522)$ & $52 \pm 82(0-220)$ & 71 & 0.03 \\
Pooled results & $116 \pm 129(0-424)$ & $59 \pm 62(0-170)$ & 56 & 0.044 \\
\hline
\end{tabular}

After 18 months follow up all symptomatic patients all of which had undergone carotid endarterectomy were alive and none had suffered renewed symptoms after surgery. All eleven asymptomatic patients were alive, but one patient had suffered a minor stroke after 16 months followup and subsequently underwent uncomplicated endarterectomy. This 
patient did not suffer from atrial fibrillation, another frequently encountered and important cause of cerebral ischemia and symptoms. Upon enrolment in the current study, the LRNC scores of this patient, as determined by the MR readers were, 0,41 and 0 for MR readers 1, 2 and 3, respectively. Histology after endarterectomy showed a LRNC score of 62 .

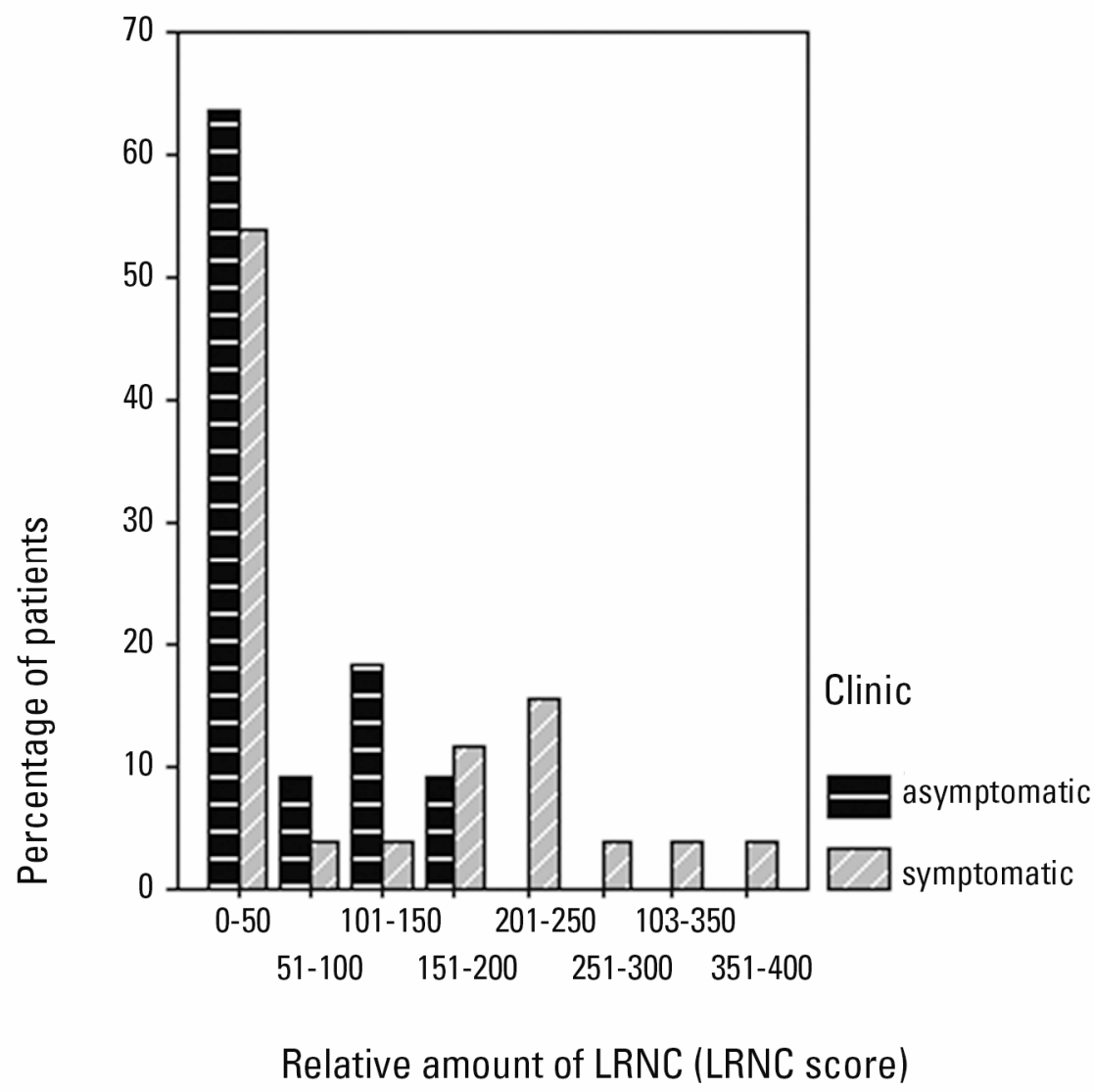

Figure 1. Distribution of LRNC score (mean LRNC score of MR readers 1, 2 and 3) in symptomatic $(n=26)$ and asymptomatic $(n=11)$ carotid patients.

In figure 2 representative cross-sectional MR images of atherosclerotic plaques of a symptomatic and asymptomatic patient with a carotid stenosis $>70 \%$ are shown. The high relative signal intensity in the T1w TFE images signifies the presence of LRNC, which is visible in the plaque of the symptomatic but not the asymptomatic patient. 

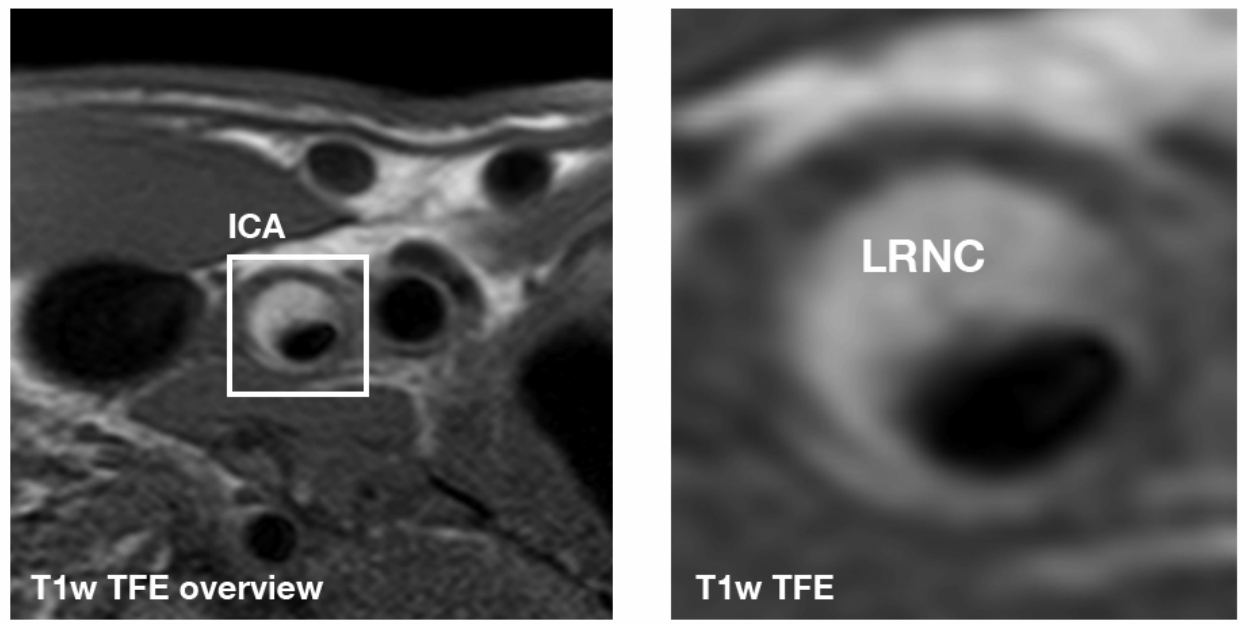

Figure 2a. T1w TFE MR image of an atherosclerotic plaque in the internal carotid artery (ICA) of a symptomatic patient. The large hyperintense region of interest within the main plaque area corresponds to LRNC.
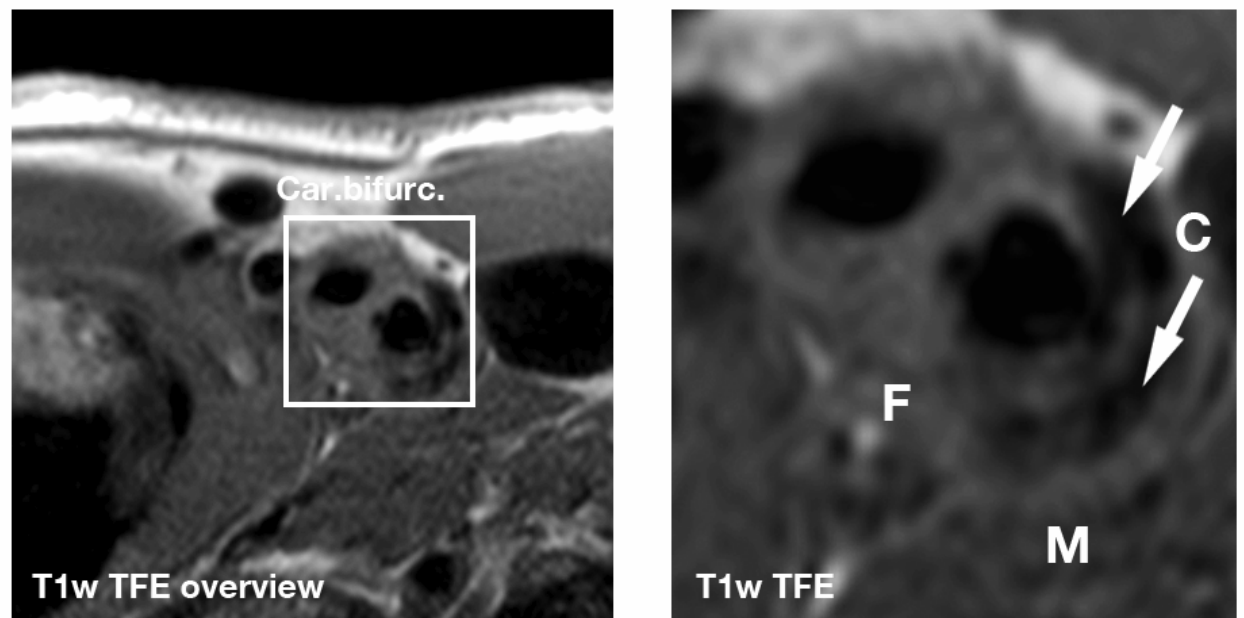

Figure 2b. T1w TFE MR image of an atherosclerotic plaque in the internal carotid artery of an asymptomatic patient. This plaque was classified as a fibro-calcific because of the multiple small hypointense areas corresponding to calcifications ( $C$, arrows). When compared to (a) there is no hyperintense signal corresponding to LRNC. Car. Bifurc: carotid bifurcation; F: fibrous tissue; M: muscle. 


\section{Discussion}

Recent studies point to the LRNC as a pivotal component of symptomatic carotid atherosclerotic plaques. Given the fact that MRI is a reliable tool for detection and quantification of LRNC we asked ourselves the question if there is a difference in LRNC size between symptomatic and asymptomatic patients with high grade carotid artery stenosis. The present study found that patients with a carotid stenosis $>70 \%$ and a recent TIA or stroke have on average a significantly larger lipid-rich necrotic core (LRNC) compared to asymptomatic patients. This finding corroborates the important role of the LRNC with regard to symptoms.

Three individual MRI readers, blinded for medical information, were highly consistent with regard to the size of LRNC which is reflected by high intra- and interreader agreement. The larger LRNC of symptomatic patients supports the idea that an atherosclerotic plaque with a larger lipid core is more prone to rupture and thus has an increased risk of causing ischemic events. Longitudinal studies are warranted to investigate the predictive value of a large LRNC.

The range in LRNC scores of symptomatic patients was much larger (0-424) compared to asymptomatic patients (0-170). However, we found considerable overlap between individual patients in the symptomatic and asymptomatic groups concerning the size of the LRNC, since a number of symptomatic patients also had a small LRNC. Investigators should be aware that plaques of asymptomatic patients might also contain LRNC. Our findings are consistent with previous histologic studies of carotid plaque specimens, ${ }^{18,19,20,21,22}$ which showed a wide range of quantities of the LRNC in both symptomatic and asymptomatic patients.

The patient in the asymptomatic group who developed symptoms after 16 months of follow-up had a small LRNC (LRNC score of sixty-two at histology) and no other characteristics typically associated with vulnerability, such as a thin or ruptured fibrous cap. The LRNC score based on the initial MR images of this patient was also low. This may indicate that patient's symptoms had a different origin. It is e.g. well recognized that plaques in the aortic arch can be responsible for thrombo-embolic events in the brain since an aortic plaque of more than $4 \mathrm{~mm}$ (aortic wall thickness)is an independent predictor for recurrent brain infarction with a 
relative risk of $3.8 .{ }^{23}$ For the same reasons it is possible that some of the symptomatic patients in the current study with low LRNC scores developed thromboemboli of other origins. Therefore, in future studies that investigate the relation between LRNC size in carotid plaque and ischemic brain events it will be important to exclude other origins of symptoms such as aortic plaque.

The importance of quantification of the size of the LRNC over time in relation to the occurrence of stroke was recently demonstrated by Takaya et $\mathrm{al}^{24}$, who followed asymptomatic carotid patients with stenosis of $50-79 \%$ after baseline MRI for a mean duration of 38 months and found a significant association with subsequent ipsilateral cerebrovascular events for patients with a $10-\mathrm{mm}^{2}$ increase in LRNC area, while no significant association with the presence of a LRNC per se was found.

Quantification of the LRNC may also be important to monitor effects of medical therapy. ${ }^{25,26}$ Some investigators already described that the use of lipid-lowering treatment significantly reduced vessel wall area ${ }^{25}$ and lipid area $^{26}$ as determined with MRI. One of the most important findings of our study is the fact that single sequence T1w TFE MRI is capable of detecting and quantifying the amount of LRNC. Compared to Zhao et $\mathrm{al}^{26}$ our method provides an easy and fast alternative to reproducibly quantify the amount of LRNC and may be less complicated because our MR readers assessed just one MR pulse sequence and just two $\mathrm{rSI}$ categories (hyperintense versus hypo- to isointense) compared to sixteen $\mathrm{rSI}$ categories by Zhao et $\mathrm{al}^{26}$.

In the present study, we quantified the relative amount of LRNC in the plaques with a dedicated scoring system. This system was introduced because it reflects not only the mean cross-sectional fraction, but also the craniocaudal extent of LRNC in carotid plaque. For example, a $24 \mathrm{~mm}$ plaque in the craniocaudal direction will have a two-fold larger LRNC score compared to a $12 \mathrm{~mm}$ plaque with the same cross-sectional LRNC fraction (e.g. area percentage per $3-\mathrm{mm}$ slice $=50$ : score is 400 vs. 200 , while mean area percentage is 50 in both cases). Future studies will need to test the hypothesis that a larger LRNC score corresponds to a higher risk of thrombo-embolic complications. 


\section{Limitations}

A limitation of the present study is that patients were assessed only by patient's history and clinical examination. No other tests were used to assure that patients were indeed asymptomatic. For future studies it would be better to obtain baseline and follow up MRI of the brain to detect and quantify ischemic lesions at start and during follow up. In addition, future studies need to assess the aortic arch as an alternative source of thromboemboli.

Furthermore, it would have been better to analyse LRNC volumes $\left(\mathrm{mm}^{3}\right)$ instead of relative amounts to have a more relevant unit for comparison with other studies. However, in the present study we lacked the means to do so. On the other hand, even though the assessment of the size of the LRNC with grids was laborious, the technique was found to be reliable in a previous study illustrated with high intra-, interreader agreement of two MR readers and high correlations with histology (unpublished data) and in the present study illustrated with high intra- and interreader agreement of three MR readers.

\section{Conclusions and clinical implications}

In conclusion, we found on average significantly larger LRNC sizes in symptomatic patients with high-grade carotid stenosis compared to asymptomatic patients, using single sequence T1w TFE MR imaging. The method we describe is fast and relatively straightforward and may therefore be useful for cross-sectional and longitudinal studies of LRNC size in relation to stroke and medical therapy. Overlap in relative amounts of LRNC between symptomatic and asymptomatic patients emphasizes the need for identification of alternative sources of thromboemboli, such as aortic arch plaques. Future studies are needed to assess the predictive value of different amounts of carotid LRNC for the prediction of TIA and stroke.

\section{Acknowledgements}

We would like to thank Mark Willemsen, M.D., for his extensive literature review on this subject. Dr. Leiner is an NWO-VENI fellow (grant number 916.46.034). 


\section{References}

1. MRC European Carotid Surgery Trial: interim results for symptomatic patients with severe (70-99\%) or with mild (0-29\%) carotid stenosis. European Carotid Surgery Trialists' Collaborative Group. Lancet. 1991;337:1235-1243.

2. Beneficial effect of carotid endarterectomy in symptomatic patients with high-grade carotid stenosis. North American Symptomatic Carotid Endarterectomy Trial Collaborators. N Engl J Med. 1991;325:445-453.

3. Randomised trial of endarterectomy for recently symptomatic carotid stenosis: final results of the MRC European Carotid Surgery Trial (ECST). Lancet. 1998;351:1379-1387.

4. Barnett HJ, Taylor DW, Eliasziw M, Fox AJ, Ferguson GG, Haynes RB, Rankin RN, Clagett GP, Hachinski VC, Sackett DL, Thorpe KE, Meldrum $H E$, Spence JD. Benefit of carotid endarterectomy in patients with symptomatic moderate or severe stenosis. North American Symptomatic Carotid Endarterectomy Trial Collaborators. N Engl J Med. 1998;339:1415-1425.

5. Rothwell PM, Eliasziw M, Gutnikov SA, Fox AJ, Taylor DW, Mayberg MR, Warlow CP, Barnett HJ. Analysis of pooled data from the randomised controlled trials of endarterectomy for symptomatic carotid stenosis. Lancet. 2003;361:107-116.

6. Endarterectomy for asymptomatic carotid artery stenosis. Executive Committee for the Asymptomatic Carotid Atherosclerosis Study. Jama. 1995;273:1421-1428.

7. Halliday A, Mansfield A, Marro J, Peto C, Peto R, Potter J, Thomas D. Prevention of disabling and fatal strokes by successful carotid endarterectomy in patients without recent neurological symptoms: randomised controlled trial. Lancet. 2004;363:1491-1502.

8. Rothwell PM, Goldstein LB. Carotid endarterectomy for asymptomatic carotid stenosis: asymptomatic carotid surgery trial. Stroke. 2004;35:2425-2427.

9. Rothwell PM, Warlow CP. Prediction of benefit from carotid endarterectomy in individual patients: a risk-modelling study. European Carotid Surgery Trialists' Collaborative Group. Lancet. 1999;353:2105-2110. 
10. Falk E, Shah PK, Fuster V. Coronary plaque disruption. Circulation. 1995;92:657-671.

11. Virmani R, Kolodgie FD, Burke AP, Farb A, Schwartz SM. Lessons from sudden coronary death: a comprehensive morphological classification scheme for atherosclerotic lesions. Arterioscler Thromb Vasc Biol. 2000;20:1262-1275.

12. Gutstein DE, Fuster, V. Pathophysiology and clinical significance of atherosclerotic plaque rupture. Cardiovasc Res. 1999;41:323-333.

13. Naghavi M, Libby P, Falk E, Casscells SW, Litovsky S, Rumberger J, Badimon JJ, Stefanadis C, Moreno P, Pasterkamp G, Fayad Z, Stone PH, Waxman S, Raggi P, Madjid M, Zarrabi A, Burke A, Yuan C, Fitzgerald PJ, Siscovick DS, de Korte $C L$, Aikawa M, Juhani Airaksinen KE, Assmann G, Becker CR, Chesebro JH, Farb A, Galis ZS, Jackson C, Jang IK, Koenig W, Lodder RA, March K, Demirovic J, Navab M, Priori SG, Rekhter MD, Bahr R, Grundy SM, Mehran R, Colombo A, Boerwinkle E, Ballantyne C, Insull W, Jr., Schwartz RS, Vogel R, Serruys PW, Hansson GK, Faxon DP, Kaul S, Drexler H, Greenland P, Muller JE, Virmani R, Ridker PM, Zipes DP, Shah PK, Willerson JT. From vulnerable plaque to vulnerable patient: a call for new definitions and risk assessment strategies: Part I. Circulation. 2003;108:1664-1672.

14. Falk E. Stable versus unstable atherosclerosis: Clinical aspects. Am Heart J. 1999;138:S421-S425.

15. Saam T, Ferguson MS, Yarnykh VL, Takaya N, Xu D, Polissar NL, Hatsukami TS, Yuan C. Quantitative evaluation of carotid plaque composition by in vivo MRI. Arterioscler Thromb Vasc Biol. 2005;25:234-239.

16. O'Brien PC. Procedures for comparing samples with multiple endpoints. Biometrics. 1984;40:1079-1087.

17. Läuter J. Exact $T$ and $F$ tests for analyzing studies with multiple endpoints. Biometrics. 1996;52:964-970.

18. Hatsukami TS, Ferguson MS, Beach KW, Gordon D, Detmer P, Burns D, Alpers $C$, Strandness DE, Jr. Carotid plaque morphology and clinical events. Stroke. 1997;28:95-100.

19. Tegos TJ, Sohail M, Sabetai MM, Robless P, Akbar N, Pare G, Stansby G, Nicolaides AN. Echomorphologic and histopathologic characteristics of unstable carotid plaques. Am J Neuroradiol. 2000;21:1937-1944. 
20. Montauban van Swijndregt AD, Elbers HR, Moll FL, de Letter J, Ackerstaff RG. Cerebral ischemic disease and morphometric analyses of carotid plaques. Ann Vasc Surg. 1999;13:468-474.

21. Seeger JM, Barratt E, Lawson GA, Klingman N. The relationship between carotid plaque composition, plaque morphology, and neurologic symptoms. J Surg Res. 1995;58:330-336.

22. Arapoglou B, Kondi-Pafiti A, Katsenis K, Dimakakos P. The clinical significance of carotid plaque haemorrhage. Int Angiol. 1994;13:323-326.

23. Atherosclerotic disease of the aortic arch as a risk factor for recurrent ischemic stroke. The French Study of Aortic Plaques in Stroke Group. N Engl J Med. 1996;334:1216-1221.

24. Takaya N, Yuan C, Chu B, Saam T, Underhill H, Cai J, Tran N, Polissar NL, Isaac C, Ferguson MS, Garden GA, Cramer SC, Maravilla KR, Hashimoto $B$, Hatsukami TS. Association Between Carotid Plaque Characteristics and Subsequent Ischemic Cerebrovascular Events. A Prospective Assessment With MRI--Initial Results. Stroke. 2006;37:818-823.

Figure 3 B, chapter 4. Histologic slice corresponding to the MR images of figure $3 A$, page 59
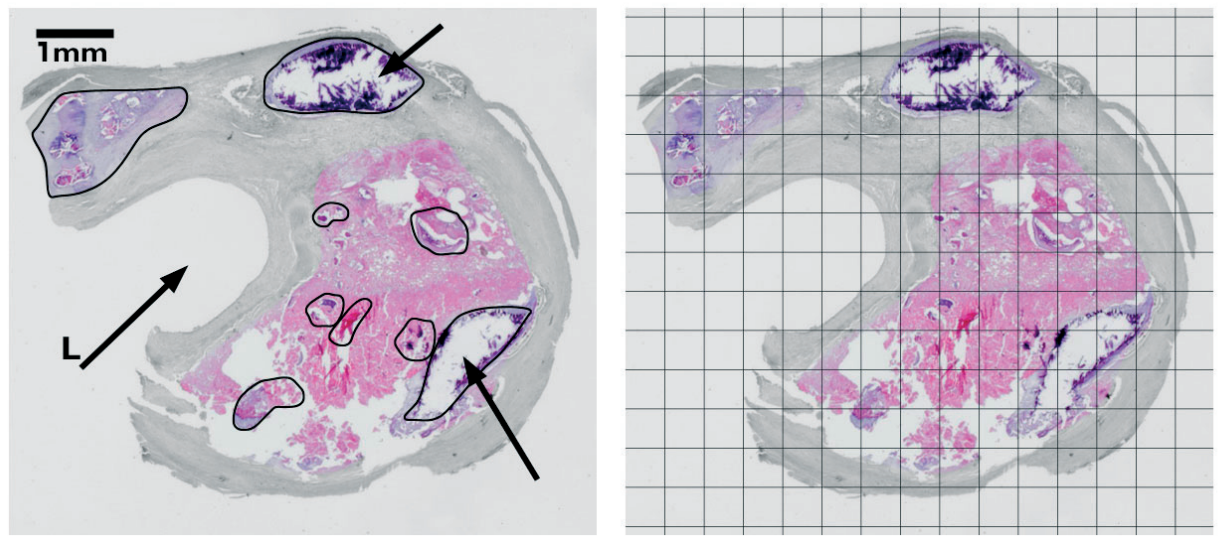

Figure 3 B. Hematoxylin-eosin stain (magnification, x6). Left: Two histology readers traced the plaque components in consensus. The ROls in this histologic slice were considered calcification, the pink region in the middle was considered intra-plaque hemorrhage and the remaining region (gray in this figure) was considered fibrous tissue. Right: Area percentages of ROls compared to the whole vessel wall area were calculated using a transparent grid (the ROls as in the left image are not shown here for esthetic reasons). 
25. Corti R, Fuster V, Fayad ZA, Worthley SG, Helft G, Chaplin WF, Muntwyler J, Viles-Gonzalez JF, Weinberger J, Smith DA, Mizsei G, Badimon JJ. Effects of aggressive versus conventional lipid-lowering therapy by simvastatin on human atherosclerotic lesions: a prospective, randomized, double-blind trial with high-resolution magnetic resonance imaging. J Am Coll Cardiol. 2005;46:106-112.

26. Zhao XQ, Yuan C, Hatsukami TS, Frechette EH, Kang XJ, Maravilla KR, Brown BG. Effects of prolonged intensive lipid-lowering therapy on the characteristics of carotid atherosclerotic plaques in vivo by MRI: a casecontrol study. Arterioscler Thromb Vasc Biol. 2001;21:1623-1629. 


\section{Chapter}

6

\section{Accumulation of ultrasmall superparamagnetic particles of iron oxide in human atherosclerotic plaques can be detected by in vivo magnetic resonance imaging}

ME Kooi, VC Cappendijk, KBJM Cleutjens, AGH Kessels, PJEHM Kitslaar, M Borgers, PM Frederik, MJAP Daemen, JMA van Engelshoven

Published in Circulation 2003;107:2453-2458

This study was financially supported by the Dutch Heart Foundation (2000.173) and Guerbet. 


\section{Abstract}

Background: One of the features of high-risk atherosclerotic plaques is a preponderance of macrophages. Experimental studies with hyperlipidemic rabbits have shown that ultrasmall superparamagnetic particles of iron oxide (USPIOs) accumulate in plaques with a high macrophage content and that this induces magnetic resonance (MR) signal changes. The purpose of our study was to investigate whether USPIOenhanced MRI can also be used for in vivo detection of macrophages in human plaques.

Methods and Results: MRI was performed on 11 symptomatic patients scheduled for carotid endarterectomy before and $24(n=11)$ and $72(n=5)$ hours after administration of USPIOs (Sinerem) at a dose of $2.6 \mathrm{mg} \mathrm{Fe} / \mathrm{kg}$. Histological and electron microscopical analyses of the plaques showed USPIOs primarily in macrophages within the plaques in 10 of 11 patients. Histological analysis showed USPIOs in 27 of $36(75 \%)$ of the ruptured and rupture prone lesions and 1 of $14(7 \%)$ of the stable lesions. Of the patients with USPIO uptake, signal changes in the postUSPIO MRI were observed by 2 observers in the vessel wall in 67 of 123 $(54 \%)$ and 19 of $55(35 \%)$ quadrants of the T2* weighted MR images acquired after 24 and 72 hours, respectively. For those quadrants with changes, there was a significant signal decrease of $24 \%$ (95\% Cl: $33 \%$ to $15 \%)$ in regions of interest in the images acquired after 24 hours, whereas no significant signal change was found after 72 hours.

Conclusions: Accumulation of USPIOs in macrophages in predominantly ruptured and rupture-prone human atherosclerotic lesions caused signal decreases in the in vivo MR images. 


\section{Introduction}

Plaque instability, which is responsible for most of the complications of atherosclerotic disease, is morphologically characterized by a preponderance of macrophages, ulceration, intra-plaque hemorrhage and thrombosis. ${ }^{1}$ Using MRI, it is possible to differentiate between lipid, fibrous, and calcified plaque components. $^{2}$

Recently, atherosclerotic plaques have been imaged by MR in hyperlipidemic rabbits using ultrasmall super paramagnetic particles of iron oxide (USPIOs). ${ }^{3.4}$ USPIOs are iron oxide nanoparticles stabilized with low molecular weight dextran with a mean diameter of $30 \mathrm{~nm}$. These relatively small particles have a much larger half-life in blood than the conventional superparamagnetic iron oxide particles (SPIO) with a mean diameter of $150 \mathrm{~nm}$. Because to their long half-life in blood, USPIOs can be taken up by macrophages in the whole body. The animal studies indicate that the USPIOs are phagocytosed by macrophages in atherosclerotic plaques, which causes a signal decrease on MR images. Additionally, a retrospective analysis of MR images of 19 patients who had originally received USPIOs for cancer staging showed signal loss in the arterial wall of 7 patients on postcontrast compared with precontrast images. ${ }^{5}$ However, in this retrospective study, no material was available for histological or electron microscopical analysis of the plaques. The aim of the present study was to investigate the feasibility of in vivo MR imaging of USPIO accumulation in human atherosclerotic plaques. Additionally, we aimed to examine the relationship between MR signal changes in plaques after USPIO administration, the amount of USPIO uptake, macrophage content, and plaque stage.

\section{Material \& Methods}

\section{Patients}

Eleven patients with symptoms of recurrent transient ischemic attacks (TIAs) or small brain infarcts and an ultrasound-proven carotid stenosis between 70 and $99 \%$ who were scheduled for carotid endarterectomy were included in this single center study. The institutional review board of the 
hospital approved the study, and written informed consent was obtained from all patients. The patients were recruited consecutively. The age of the patients was $64 \pm 9$ (mean $\pm \mathrm{sd}$ ) years and 9 of the patients were male. The procedures followed were in accordance with the institutional guidelines. Adverse events, defined as any untoward medical occurrence in a patient administered Sinerem, observed from the start of USPIO infusion (Sinerem, Guerbet) until the end of post-USPIO MRI (24 to 72 hours after the start of the infusion) were recorded in case report forms. No adverse events related to Sinerem were recorded in these eleven patients. Additional information about clinical safety can be found in the study by Sigal et al. ${ }^{6}$

\section{Magnetic Resonance Imaging}

The carotid atherosclerotic plaques of the patients were imaged in vivo on a 1.5-T whole-body scanner (Intera, Philips Medical Systems, Best, the Netherlands) using a surface radio frequency coil with a diameter of $47 \mathrm{~mm}$. The patients were examined immediately before and $24(n=11)$ and 72 hours $(n=5)$ after administration of USPIOs (Sinerem ${ }^{\circledR}$, Guerbet, Paris), which were administered at $2.6 \mathrm{mg} \mathrm{Fe} / \mathrm{kg}$ at an infusion rate of $4 \mathrm{~mL} / \mathrm{min}$. The infusion time was typically 30 minutes. After a survey to determine the position of the carotid plaque, the following MRI sequences were used: (1) T1-weighted (T1w) gradient-recalled-echo, TR/TE: 41-44/ 8.0-9.2 ms; flip angle: $25^{\circ}$; (2) T2* $\mathrm{w}$ gradient-echo (GE), TR/TE: 1 heart beat/20 ms; flip angle: $40^{\circ}$; and (3) proton-density-weighted turbo spinecho (PDw TSE), TR/TE: 2-3 beats/20 ms; echo train length: 5 . Other parameters were transversal slices; field of view, $70 \times 56 \mathrm{~mm}$; matrix size, 208 to 256x166 to 205; and slice thickness, $5.0 \mathrm{~mm}$. A frequency-selective prepulse for fat suppression was used in the T2* $W$ GE and the PDW TSE sequence. Note that the T1w GE images also contain some T2* weighting. Because the plaque was usually located just above the carotid bifurcation, for each sequence, typically 1 to 2 images of plaque in the common carotid artery and 3 to 4 images of plaque the internal carotid artery were obtained. Occasionally, the plaque extended in one image of the external carotid artery. 


\section{Image evaluation}

Pre- and post-USPIO MR images were matched, using the carotid artery bifurcation as a marker. The reading of the MR images was done irrespective of the histological results by two readers by consensus (JvE and VC). The readers judged all images of atherosclerotic plaque with respect to signal changes in the vessel wall in the postcontrast compared with the corresponding precontrast image and the quadrant of the vessel wall in which these changes were present. Additionally, the relative signal intensity ( $\mathrm{rSI}$ ) in the quadrants with signal changes was determined by dividing the signal intensity of operator-defined regions of interest by that of surrounding muscle tissue. The operator who defined the regions of interest was unaware of the histological results. Signal changes were quantified by calculating the rSI ratio, which was defined as the quotient of the $\mathrm{rSI}$ in post- and pre-USPIO MRI. With the first 5 patients, the optimal delay for post-USPIO MRI was determined. In order to be able to compare the performance of the T2* ${ }^{*}$ GE with the PDw TSE sequence, only those carotid segments of which images of both sequences were available were included for data analysis.

\section{Histology and immunohistochemistry}

After surgery, the intact carotid artery segments were formalin-fixed, sectioned in 5-mm transversal slices (in plane with the MRI slices), decalcified for $30 \mathrm{~min}$ in $10 \%$ formic acid in PBS, and embedded in paraffin. Subsequently, $4-\mu \mathrm{m}$ sections were subjected to histo-immunological analysis of plaque phenotype (HE staining), macrophage content (CD68 immunostaining) and USPIO uptake (PERL's iron staining). Typically, for each patient, 2 tissue slices from the common carotid artery, 3 from the internal carotid artery and, occasionally, 1 from the external carotid artery were obtained. In addition, a total of 10 atherosclerotic carotid artery samples consisting of 56 tissue slices derived at autopsy from patients who did not receive USPIO were analyzed for endogenous iron staining. Sections subjected to CD68 staining were pre-treated with $0.1 \mathrm{~mol} / \mathrm{L}$ pepsin and subsequently incubated with monoclonal CD68 mouse antibody (DAKO 1:100), biotinylated swine-anti-mouse antibody (1:250, Amersham Life Science) and alkaline phosphatase-coupled ABC-reagent (1:200, Dako). 

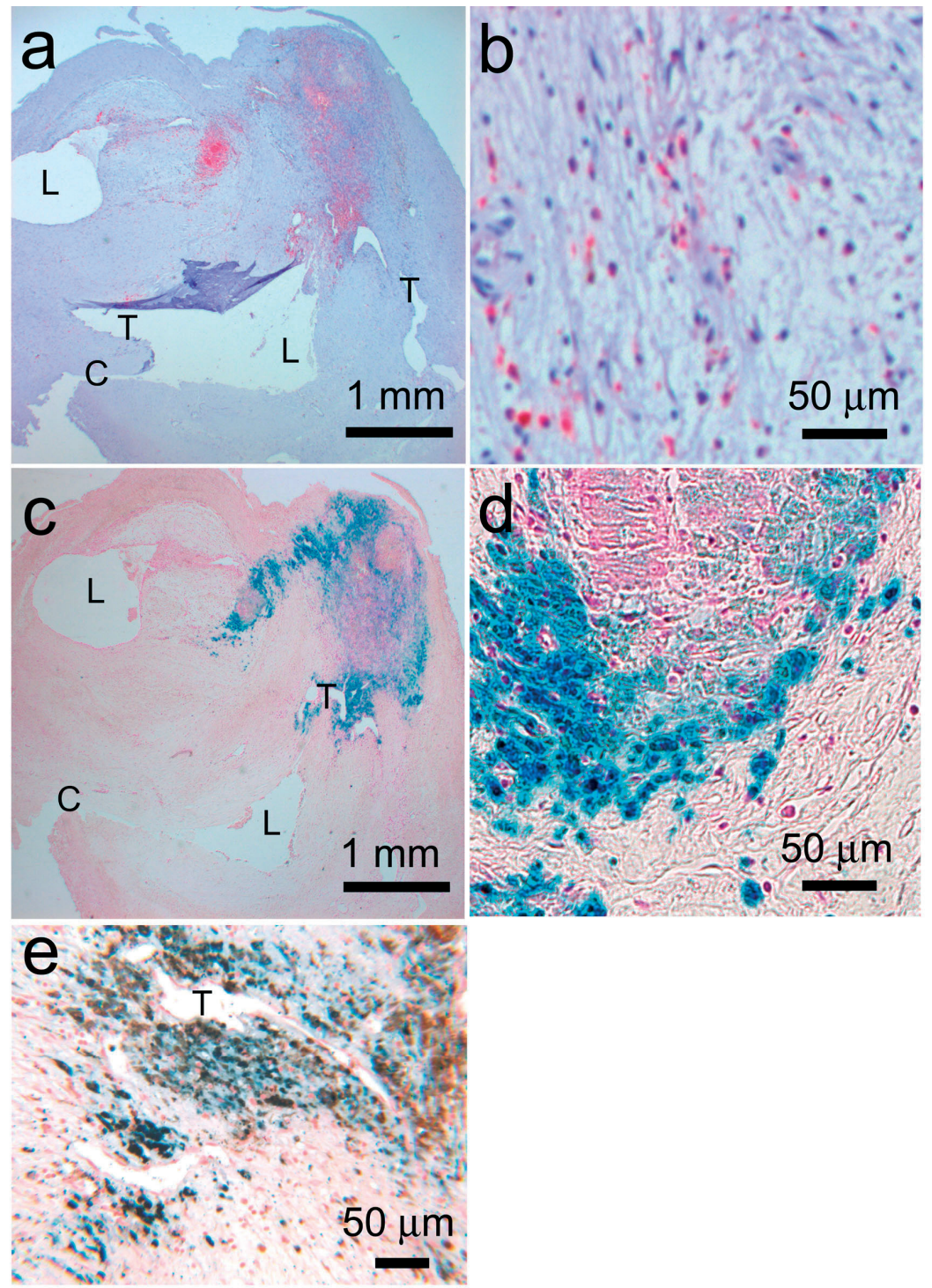

Figure 1. CD68 ( $a$ and b), PERL's (c and d), and CD68/PERL's (e) double staining of the endarterectomy specimen of a patient who received USPIOs. Double staining showed colocalization of macrophages and PERL's iron staining. The red coloring ( $a$ and $b$ ) and brown coloring (e) are indicative of macrophages, and the blue coloring (c, $d$ and e) is indicative of the accumulation of USPIOs. The double lumen is indicated with $\mathrm{L}$, the surgical cut with $\mathrm{C}$ and tears with $\mathrm{T}$. 

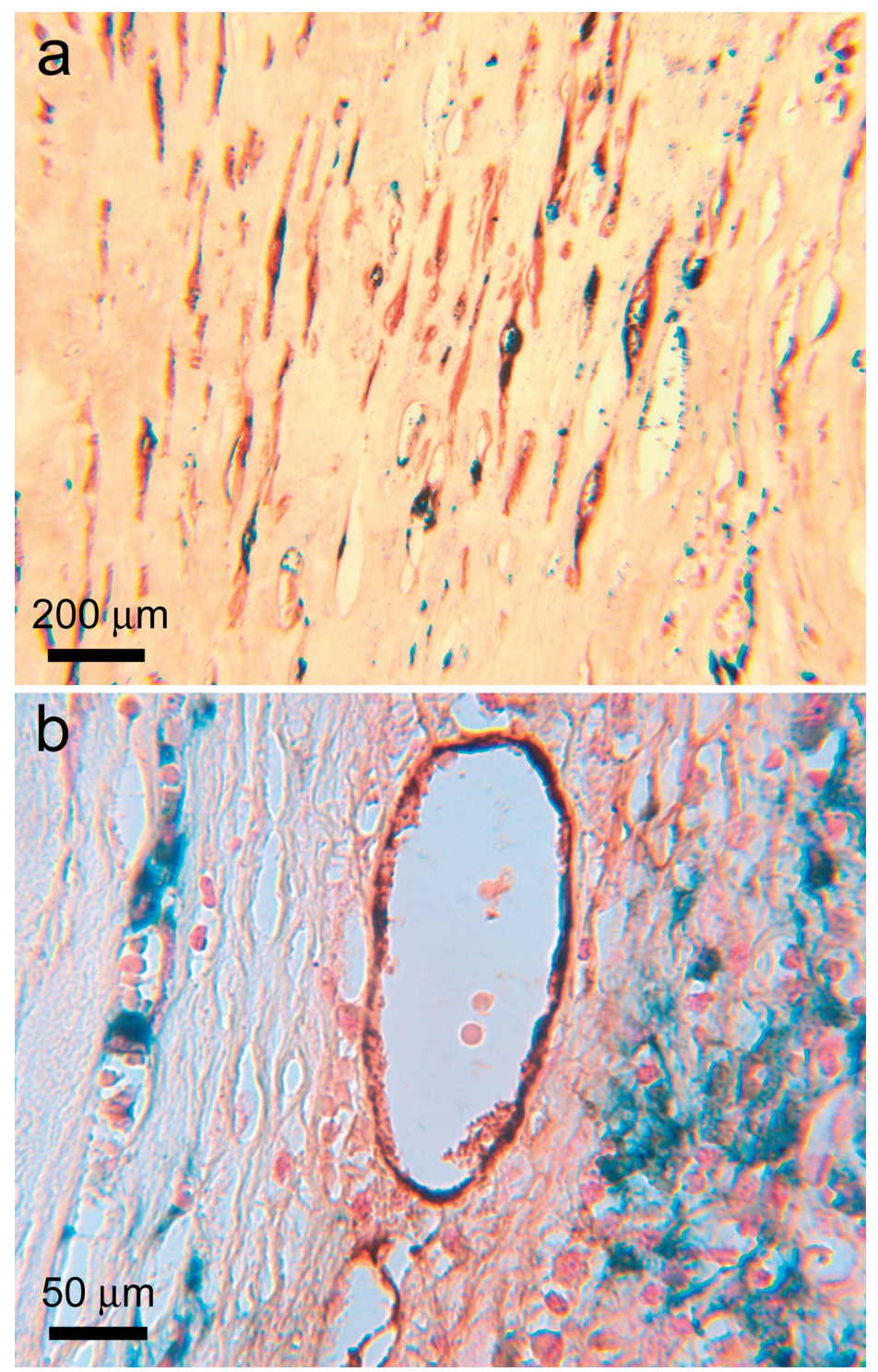

Figure 3. USPIO uptake by vascular smooth muscle cells, myofibroblasts, and endothelial cells. Double staining of endarterectomy specimen of a patient who received USPIOs showed colocalization of $\alpha$-smooth cell actin (ASMA) and PERL's iron staining (a) and CD31 and PERL's iron staining (b). Positive ASMA and CD31 immunostaining is represented in brown, whereas PERL's iron staining (indicative for the accumulation of USPIOs) results in the formation of a blue precipitate. 
Alkaline phosphatase activity was visualized using the Alkaline Phosphatase Kit I (Vector). Sections were counterstained with hematoxylin.

USPIO uptake (PERL's iron staining) and the number of macrophages (CD68-positive cells) in the four quadrants were assessed by one investigator $(\mathrm{KC})$ on a scale from 0 to 4 , with 0 indicating no; 1 , hardly any; 2, some; 3, many; and 4, very many iron/CD68-positive cells. In addition, for each tissue section, plaque stage was independently determined by two investigators (KC and R. J. van Suylen, University Hospital Maastricht, Maastricht, The Netherlands) according to the morphological criteria of the American Heart Association." Stable lesions (type IV and V) are characterized by an intact and thick fibrous cap. These plaques contain either a large lipid core (type $\mathrm{Va}$ ), calcification (type $\mathrm{Vb}$ ) or fibrous tissue (type $\mathrm{Vc}$ ). A disrupted fibrous cap and the presence of a thrombus characterize the ruptured lesion (type VI). Type Va lesions with a very thin fibrous cap were considered as rupture-prone lesions.

To verify USPIO uptake by macrophages and to identify additional cell types containing USPIOs, carotid sections derived from patients who did receive USPIOs were double stained for Perl's iron and CD68, a-smooth muscle actin (mouse monoclonal anti-ASMA antibody, Sigma 1:500) as a marker of vascular smooth muscle cells and myofibroblasts and CD31 (mouse monoclonal anti-CD31 antibody, DAKO 1:100) as a marker of endothelial cells.

Electron microscopy and Energy dispersive-X-ray analysis of Perl's positive regions

For two of the specimens, tissue fragments of about $1 \mathrm{~mm}^{3}$ were fixed overnight in a $2.5 \%$ glutaraldehyde solution. After several washes, tissue fragments were postfixed in $1 \%$ osmium tetroxide solution and routinely dehydrated and embedded in epoxy resin. Semi-thin $(1-\mu \mathrm{m})$ serial sections were subjected to Perl's iron staining. Subsequently, ultra-thin sections from PERL's positive regions were mounted on Formvar (1595E, Merck)coated 75 mesh Copper grids and counterstained with uranyl acetate and lead citrate before analysis in a Philips CM12 transmission electron microscope. Subsequently, EDX spectrometry of the observed dense bodies was performed essentially as described previously. ${ }^{7.8}$ 


\section{Statistical analysis}

A one-sample t-test was used to test whether the rSI ratios of the MR images of each sequence were significantly $(P<0.05)$ different from unity. The optimal delay for post-USPIO MRI was taken to be the time with the largest significant deviation of the $\mathrm{rSI}$ ratios from unity. A $\mathrm{X}^{2}$ test was used to test whether the proportions of USPIO uptake in stable versus ruptured and rupture-prone sections were significantly different.

\section{Results}

\section{Histology and electron microscopy}

The time between USPIO administration and surgery was 21 days for one patient, and therefore this patient was excluded from further analysis. For the remaining 10 patients time between administration and surgery was $4.7 \pm 3.0$ (mean \pm standard deviation; range 2 to11) days. Perl's staining showed USPIO accumulation in the plaques of 9 of these 10 patients. Eight of the patients with USPIO accumulation showed a considerable USPIO uptake (total iron score $\geq 7$ in all available quadrants), whereas 1 showed some uptake (total iron score of 4 in all available quadrants). The time between administration of USPIOs and surgery was only three days for the patient that showed no USPIO uptake. Additionally, also the plaque of the patient with the delay time of 21 days showed USPIO uptake. PERL's staining of the carotid atherosclerotic plaques of the 10 control patients who did not receive USPIOs showed no endogeneous iron staining. In contrast, CD68 (macrophages)/ PERL's iron (USPIO) double staining of carotid artery tissue sections derived from patients who did receive USPIOs, showed extensive intracellular, granular localization of ironcontaining particles in the cytoplasm of macrophages, especially in cells in the vicinity of small capillaries (Figure 1, page 94).

This finding was additionally substantiated by electron microscopical analysis of PERL's positive regions in plaque material of two patients. Figure 2a shows a typical electron microscopy image indicating the presence of numerous USPIO particles in a single macrophage. The observed particle size corresponds to the size of Sinerem. Electron 

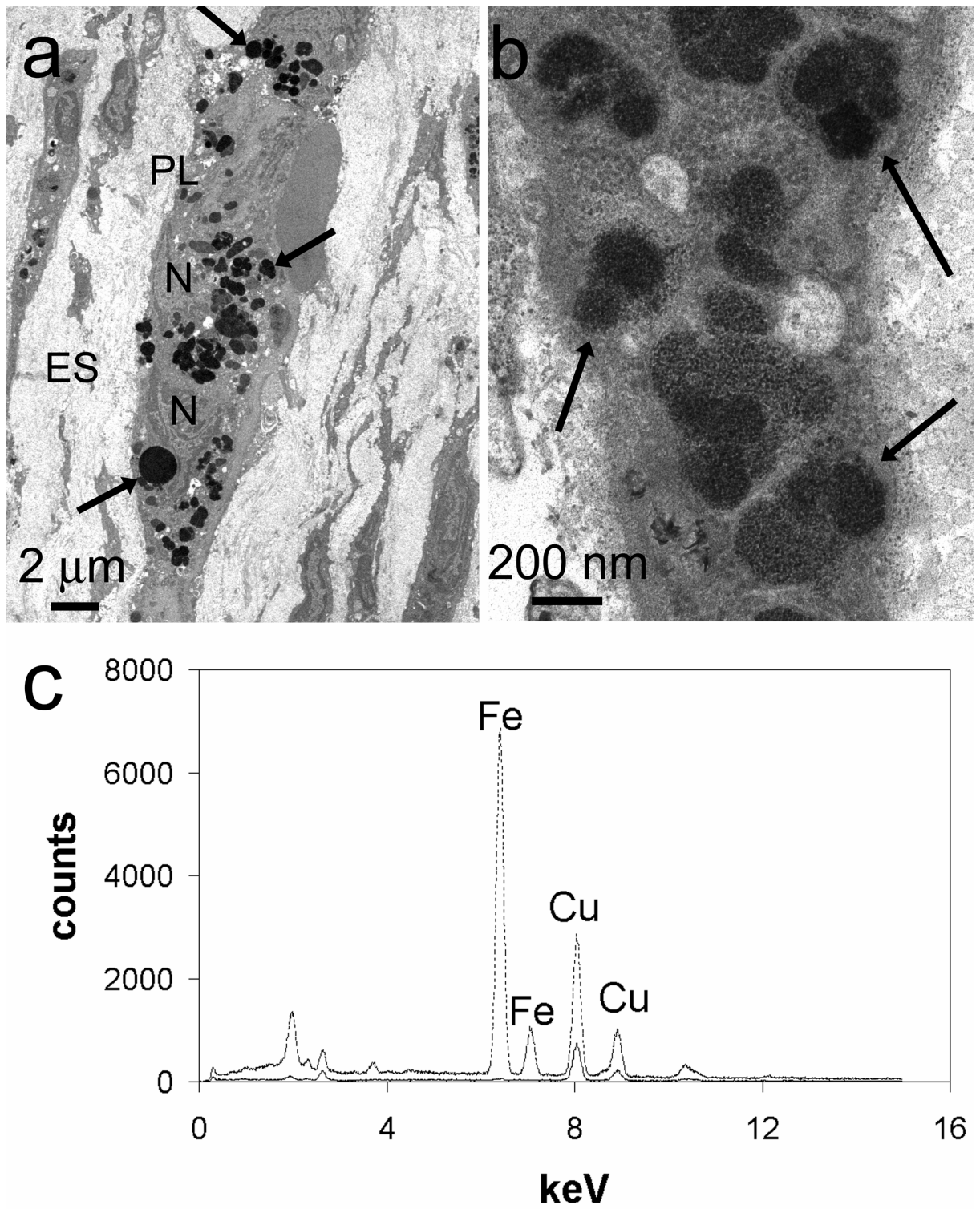

Figure 2. Electron microscopy and EDX analysis of PERL's positive regions of an endarterectomy specimen of a patient who received USPIOs. a, Presence of numerous USPIO particles in a single macrophage (arrows). The plasma membrane is indicated with $\mathrm{PL}$, the cell nucleus with $\mathrm{N}$, and the extracellular space with ES. b, Detail of phagosomes in macrophages containing clusters of iron particles (arrows). c, EDX spectrum of particles in macrophages (dotted line). It is shown that these particles contain a large amount of iron. The solid line corresponds to an EDX spectrum of a reference area. 
microscopy showed an intracellular granular iron distribution in phagosomes of macrophages. In Figure $2 \mathrm{~b}$, it can be observed that $a$ single phagosome contained many USPIO particles. Energy-dispersive $\mathrm{X}$-ray (EDX) analysis of the dense particles showed that these particles contain a large amount of iron, additionally confirming that they represent USPIOs (Figure 2c), whereas in the PERL's negative regions, no iron could be observed. Next to USPIO-positive macrophages, negative macrophages were also observed.

Although most USPIO staining was confined to macrophages, incidentally we observed ASMA/PERL's iron and CD31/PERL's iron double staining, which is indicative for limited USPIO uptake by smooth muscle cells, myofibroblasts, and endothelial cells (Figure 3, page 95).

In total, 50 histological sections were obtained. The stage of the plaques in each section was compared with the uptake of USPIO (PERL's positive regions). Only $7 \%$ ( 1 of 14 ) of the stable lesions showed USPIO uptake, whereas this number was $75 \%$ (27 of 36 ) for the rupture-prone and ruptured lesions. These proportions were significantly different $(p<0.0001)$. From the plaques of every patient, at least two sections were ruptured or rupture-prone.

In total, 235 quadrants were available for CD68 immuno(macrophages) and PERL's iron reading. Note that occasionally sections consisted of material from both the carotid internal and external artery (8 quadrants), whereas in other sections $<4$ quadrants were available because of incomplete intima removal during endarterectomy. Of these 235 quadrants, 86 showed PERL's positive regions, indicating the uptake of USPIO. All quadrants that were PERL's positive were also CD68 positive, but not vice versa. PERL's positive cells were present in 3 of 35 quadrants with hardly any, 17 of 55 with some, 45 of 80 with many, and 21 of 39 with very many CD68-positive cells.

\section{Magnetic Resonance Imaging}

The $\mathrm{rSI}$ of the vessel wall on T2* $\mathrm{W}$ GE images acquired 24 hours after administration of USPIOs was decreased in comparison with the $\mathrm{rSI}$ on pre-USPIO images. From a comparison with histology, it becomes apparent that the decrease in rSI was confined to parts of the vessel wall that showed uptake of USPIO (Figure 4). Of the T2* ${ }^{*}$ GE images of 
patients showing USPIO uptake acquired with a delay time of 24 and 72 hours, 67 of $123(54 \%)$ and 19 of $55(35 \%)$, respectively, showed signal changes. After 24 hours, the mean rSI ratio of the quadrants with signal changes was $0.76(95 \% \mathrm{Cl}, 0.67$ to 0.85$)$, indicating a signal decrease of $24 \%$. The mean rSI ratio after 72 hours was not significantly different from unity $(P<0.05)$. In 4 images, a dark band adjacent to the lumen was observed in the post-USPIO MRI. Images with this or other artifacts were excluded from additional analysis.
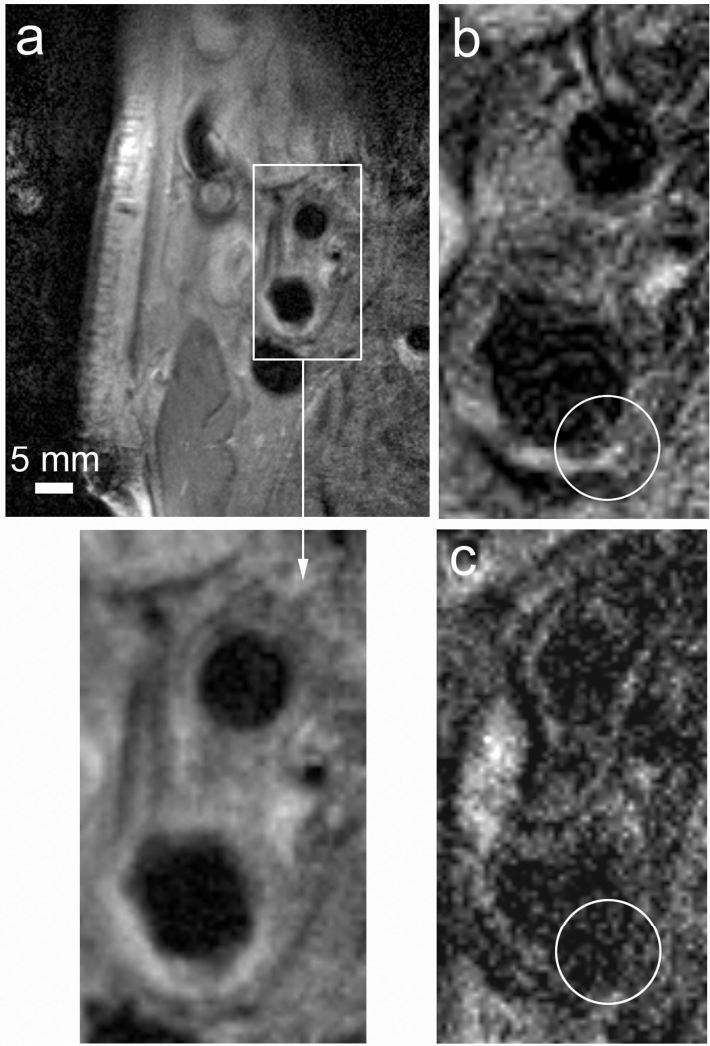

Figure 4. Corresponding in vivo MR images of the external (above) and internal (below) carotid artery. a, PDw turbo spin-echo MR image; $b, T 2{ }^{*} w$ gradient-echo MR image before administration of USPIOs; $c, T 2 * w$ gradient-echo MR image 24 hours after administration of USPIOs. A signal decrease can be observed on the post-contrast T2* $\mathrm{w}$ MR image (circle) in the part of the vessel wall that showed a large number of Fe-positive cells in the corresponding histological section. PERL's reading in the lower quadrants was many and very many iron positive cells, respectively, whereas it was hardly any for both the upper quadrants. 


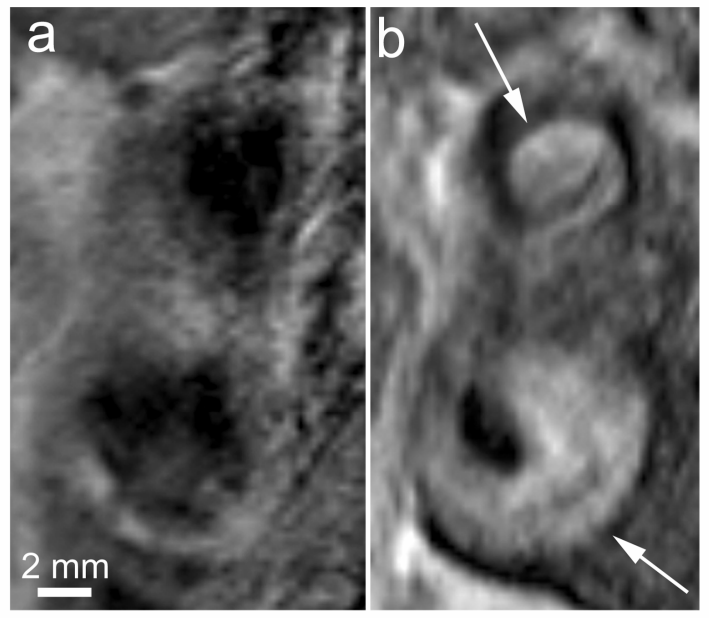

Figure 5. Corresponding in vivo T1w gradient-echo MR image of the external (above) and internal (below) carotid artery before (a) and 24 hours after (b) administration. On the postcontrast image, a narrow band adjacent to the hyperintense signal of the lumen is observed (arrows).

In the T1w GE images, 24 hours after administration, in most cases the lumen was hyperintense. In these images, a narrow hypointense band adjacent to the hyperintense signal was observed (Figure 5); This band was not clearly confined to either the vessel wall or the lumen. This effect also occurred in other arteries. In the images acquired 72 hours after administration of USPIOs, the signal intensity of the lumen had decreased, but in some cases this artifact was still observed. Because this phenomenon also led to a signal decrease in the vessel wall, it was impossible to quantify signal changes because of the uptake of USPIOs in the T1w GE MR images.

Of the PDW TSE images of patients showing USPIO uptake acquired with a delay time of 24 and 72 hours, 51 of $123(41 \%)$ and 15 of $55(27 \%)$, respectively, showed signal changes. However, the mean rSI ratios for these images, after both 24 and 72 hours, of the patients that showed uptake of USPIOs, were not significantly different from unity. 


\section{Discussion}

The results of the present study show that the tested USPIOs accumulate predominantly in macrophages in ruptured and rupture-prone human atherosclerotic lesions and that this can induce significant signal changes in the in vivo $T 2 * W$ GE MR images acquired 24 hours after intravenous administration of USPIOs. The implications of this study could be vast, because it indicates that USPIO-enhanced MRI is a promising noninvasive method to identify high-risk plaques.

USPIO-enhanced plaque imaging could be combined with information about plaque composition obtained from non-enhanced MR plaque imaging. High-resolution MRI has a lot of potential for non-invasive in vivo plaque characterization. ${ }^{9,10}$ Therefore, one might be able to obtain information about plaque inflammation and composition using a single modality, which will be extremely valuable for the identification of patients at risk.

The composition and stage of atherosclerotic plaque rather than the severity of stenosis have emerged as being the most important determinants for the development of acute ischemic symptoms. ${ }^{11}$ These symptoms often occur as a result of erosion or uneven thinning and rupture of the fibrous cap. Lesions at risk for rupture usually show accumulation of macrophages. ${ }^{12}$ The tested USPIO contrast agent (Sinerem) is a relatively new contrast agent which is used for MR lymphography, where the accumulation of this contrast agent in macrophages reduces the signal intensity of normal functioning nodes attributable to the $\mathrm{T}^{*}$ and $\mathrm{T} 2$ shortening effects of USPIOs. ${ }^{13}$

The exact route by which USPIOs enter the plaques is still unknown. However, the profound uptake of USPIOs by macrophages surrounding newly formed capillaries and the observation that USPIOs incidentally are located in endothelial cells suggests that the USPIOs may enter the plaque via transcytosis.

In the present study, electron microscopy showed unambiguously clustering of USPIO particles in phagosomes of macrophages. At high concentrations, USPIO decreases the signal intensity on $\mathrm{T}^{*}$ weighted images attributable to a shortening of $\mathrm{T} 2$ and $\mathrm{T} 2^{*}$. Moreover, clustering of 102 
iron oxide particles in tissue may lead to additional T2* shortening.$^{14}$ This study showed that the USPIO uptake induced significant signal decreases in the in vivo T2* $\mathrm{w}$ GE MR images acquired 24 hours after administration of USPIOs but not in the images obtained after 72 hours. This suggests an active process of uptake and washout and therefore imaging 24 hours after USPIO administration is preferred above imaging after 72 hours.

The administration dose that can be used for human studies is much lower than the dose administrated in experimental animal models. Ruehm et $\mathrm{al}^{3}$ used a very high dose, namely $56 \mathrm{mg} / \mathrm{kg}$, in hyperlipidemic rabbits. Schmitz et al showed, again in a study with hyperlipidemic rabbits, that at a dose of $11 \mathrm{mg} \mathrm{Fe} / \mathrm{kg}$ compared to $2.8 \mathrm{mg} \mathrm{Fe} / \mathrm{kg}$ many more images showed a focal signal loss in the aortic wall $(31 \pm 11 \%$ vs. $3 \pm 5 \%) .{ }^{4}$ In the present human study, a similar dose as in MR lymphography of $2.6 \mathrm{mg}$. $\mathrm{Fe} / \mathrm{kg}$ was used. An advantage of human studies is that the half-life of USPIOs in blood is much larger than in rabbits. The half-life of USPIOs in blood in humans is about 30 hours, while this is about 6 hours for rabbits. ${ }^{4}$ This longer half-life will allow for more time for the uptake of the iron oxide particles by macrophages of plaques. Another difference is that in animal studies, usually a longer imaging time can be used.

The present study has several limitations. Firstly, we experienced difficulties matching the $4.5-\mathrm{mm}$-thick MRI slices with the $4-\mu \mathrm{m}$-thick histological sections and could therefore not correlate signal changes in the MR images with USPIO uptake in the corresponding histological sections. Second, it is well known that GE sequences are most sensitive for iron detection, but unfortunately $\mathrm{T} 2{ }^{*} \mathrm{w}$ GE images have a relatively low signal to noise ratio. This has led to a somewhat limited image quality of the $T 2{ }^{*} \mathrm{~W}$ GE images in the present study. This needs to be improved for accurate detection of USPIO accumulation in human plaques by reducing the spatial resolution, by using other pulse sequences, by using a phasedarray carotid coil, or by using higher magnetic field strength. Third, the time interval between USPIO administration and surgery was $4.7 \pm 3.0$ days, whereas ideally this should be constant. Another complexing factor may be that in the post-USPIO T1w GE and some of the T2* $w$ GE MR images, a dark band adjacent to the hyperintense signal of the lumen was observed. In T1w GE images, the signal of the lumen is hyperintense 24 hours after USPIO administration because of the T1-shortening effect of a low 
concentration of USPIOs in the vascular space. This band was not clearly defined to either the lumen or the vessel wall. The same phenomenon was also observed by Schmitz et $\mathrm{al}^{,}{ }^{4}$ who could not explain it as USPIO accumulation because iron was not demonstrated in corresponding histological sections. Images with this artifact were excluded from additional analysis.

Finally, a limitation of USPIO-enhanced MRI may be that the postcontrast imaging time needs to be rather long to allow uptake of iron particles by macrophages. Furthermore, we feel it is important to compare postcontrast with corresponding precontrast images in order to differentiate signal reductions attributable to USPIO uptake from other effects. For example, plaque calcification and blood degradation products can also have strong susceptibility effects and therefore cause signal loss. However, in contrast to signal loss due to USPIO uptake, these tissues will already cause signal loss in the precontrast images.

From this proof of concept study, we conclude that the tested USPIOs accumulate predominantly in macrophages in ruptured and rupture-prone human atherosclerotic plaques. This accumulation induces signal decreases in the postcontrast MR images. Because a preponderance of macrophages is an important feature of a high-risk plaque, USPIOenhanced $\mathrm{MRI}$ is a promising method for the in vivo differentiation between low- and high-risk plaques. Larger studies are warranted to confirm these early encouraging results.

\section{Acknowledgements}

This work was supported by grants from the Dutch Heart Foundation (2000.173) and Guerbet, Paris, France. We thank Dr. R.J. van Suylen (University Hospital Maastricht, Maastricht, The Netherlands) for determining plaque stage of the histological tissue sections. 


\section{References}

1. Stary $H C$, Chandler $A B$, Dinsmore $R E$, et al. A definition of advanced types of atherosclerotic lesions and a histological classification of atherosclerosis: a report from the Committee on Vascular Lesions of the Council on Arteriosclerosis, American Heart Association. Arterioscler Thromb Vasc Biol. 1995;15:1512-31.

2. Shinnar M, Fallon JT, Wehrli S, et al. The diagnostic accuracy of ex vivo MRI for human atherosclerotic plaque characterization. Arterioscler Thromb Vasc Biol. 1999;19:2756-61.

3. Ruehm SG, Corot C, Vogt $P$, et al. Magnetic Resonance Imaging of Atherosclerotic Plaque With Ultrasmall Superparamagnetic Particles of Iron Oxide in Hyperlipidemic Rabbits. Circulation. 2001;103:415-422.

4. Schmitz SA, Coupland SE, Gust R, et al. Superparamagnetic iron oxideenhanced MRI of atherosclerotic plaques in Watanabe hereditable hyperlipidemic rabbits. Invest Radiol. 2000;35:460-71.

5. Schmitz SA, Taupitz M, Wagner S, et al. Magnetic resonance imaging of atherosclerotic plaques using superparamagnetic iron oxide particles. J Magn Reson Imaging. 2001;14:355-61.

6. Sigal R, Vogl T, Casselman J, et al. Lymph node metastases from head and neck squamous cell carcinoma: MR imaging with ultrasmall superparamagnetic iron oxide particles (Sinerem MR): results of a phase-III multicenter clinical trial. Eur Radiol. 2002;12:1104-13.

7. Hofer F, Pabst MA. Characterization of deposits in human lung tissue by a combination of different methods of analytical electron microscopy. Micron. 1998;29:7-15.

8. Drent M, Bomans PH, Van Suylen RJ, et al. Association of man-made mineral fibre exposure and sarcoidlike granulomas. Respir Med. 2000;94:815-20.

9. Fayad ZA, Fuster V. Clinical imaging of the high-risk or vulnerable atherosclerotic plaque. Circ Res. 2001;89:305-16.

10. Yuan C, Mitsumori LM, Beach KW, et al. Carotid atherosclerotic plaque: noninvasive MR characterization and identification of vulnerable lesions. Radiology. 2001;221:285-99.

11. Falk E, Shah PK, Fuster V. Coronary plaque disruption. Circulation. 1995;92:657-71. 
12. Ross R. Atherosclerosis: an inflammatory disease. $\mathrm{N}$ Engl J Med. 1999;340:115-26.

13. Anzai Y, Prince MR. Iron oxide-enhanced MR lymphography: the evaluation of cervical lymph node metastases in head and neck cancer. JMRI. 1997;7:75-81.

14. Chambon $C$, Clement $O$, Blanche $A L$, et al. Superparamagnetic iron oxides as positive MR contrast agents: in vitro and in vivo evidence. Magnetic Resonance Imaging. 1993;11:509-519. 


\section{Chapter}

7

\section{General discussion}

MRI of atherosclerosis -

Identification and quantification of carotid plaque components 
Atherosclerosis is one of the leading causes of death in the industrialized society. Atherosclerotic vessel wall changes start early in life and progress during ageing. The earliest atherosclerotic lesion is the fatty streak, which may progress to intermediate and advanced lesions. These lesions contain increasing amounts of extracellular lipid and an evolving necrotic core covered by a fibrous cap that separates the blood from the thrombogenic plaque contents. ${ }^{1}$ Plaque vulnerability is determined by its composition, e.g. the presence of a large lipid-rich necrotic core, rather than the degree of vascular stenosis. ${ }^{2,3}$ Rupture of vulnerable atherosclerotic plaques is the predominant underlying process in the pathogenesis of acute cardiovascular syndromes such as stroke and heart attack. Current clinical practice lacks non-invasive diagnostic tests for atherosclerotic plaque assessment and therefore luminography, like x-ray, MR or CT angiography, is still the most prominent imaging tool for cardiovascular disease. For carotid disease luminography proofed to be important to determine treatment strategy (endarterectomy versus medical treatment alone). ${ }^{4,5,6,7,8}$ However, luminography cannot recognize expansive remodeling of the vessel wall, which means that an atherosclerotic plaque can be present while normal lumen is diagnosed by luminography. ${ }^{9,10}$ Therefore, it is very important to develop imaging techniques, which can visualize the various components of atherosclerotic plaque. These techniques may be used to monitor progression and regression of disease during therapy and may aid in the selection of the best treatment for individual patients.

Assessment of the lipid-rich necrotic core in atherosclerotic plaque with $M R I$

In the current thesis the role of MRI as a potential imaging modality for atherosclerosis in symptomatic patients with carotid stenosis was studied. Several research groups demonstrated the ability of ex vivo ${ }^{11,12,13}$ and in vivo $^{14,15,16,17}$ multisequence MRI to identify the various plaque components. The most important goal of atherosclerotic plaque imaging is to identify the vulnerable aspects of the plaque. One of these aspects is the size of the lipid-rich necrotic core (LRNC) in the plaque. ${ }^{2,3,18,19,20,21}$ This LRNC is defined as a lipid core inside the plaque with or without intra-plaque hemorrhage. It has been suggested ${ }^{22}$ that the presence of hemorrhage in the lipid-rich 
necrotic core (LRNC) is important, because intra-plaque hemorrhage may be a stimulus for plaque growth. At the start of our studies intra-plaque hemorrhage was considered as a difficult component to image. $15,23,24$ Furthermore, up to then the literature lacked a comprehensive and systematic strategy for standardized MR image assessment of atherosclerotic plaque components. Therefore, we tested a new MR pulse sequence ("T1 weighted (w) TFE") for intra-plaque hemorrhage, ${ }^{25}$ and developed an algorithm ${ }^{26}$ to assess the optimal combinations of five MR pulse sequences and determined cutoff points for the identification of the main plaque components (calcification, lipid core, intra-plaque hemorrhage and fibrous tissue).

In the initial study ${ }^{25}$ intra-plaque hemorrhage was detected as hyperintense (compared to surrounding muscle tissue) region in the vessel wall on T1w TFE images. This study showed very promising results with a detection rate of intra-plaque hemorrhage of $80 \%$ and high interobserver agreement. These results were important, because with the high in-plane resolution smaller areas with intra-plaque hemorrhage could be detected than previously described by Moody et $\mathrm{al}^{27}$. Another important observation of our initial study ${ }^{25}$ was that for the prediction of intra-plaque hemorrhage the hyperintense signal should be located in the bulk of the plaque instead of at the borders of the plaque or in a marginal thickened vessel wall since all false positive results of hemorrhage in the T1w TFE images with high signal intensity were located outside the bulk of the plaque.

A young age of intra-plaque hemorrhage may be an important forbode of thrombo-embolic events. In literature it is reported ${ }^{28,29}$ that the signal intensity of intra-plaque hemorrhage changes dependent on the age of hemorrhage and this may have a negative influence on the performance of MRI of this component. However, a human study of the exact time relation of signal intensity on MR images and first clinical symptoms will logistically and techniquely be very challenging because MRI will often be not available at once and many factors will influence the signal intensity. Sustained high signal intensity on T1w TFE images can for instance be caused by impaired maturation of intra-plaque hemorrhage $\mathrm{e}^{30}$ and also by capillary rebleeds ${ }^{31}$ within the plaque. Unlike experimental animal studies, these factors cannot be controlled in in vivo human studies. In our initial 
study ${ }^{25}$ the detection rate of intra-plaque hemorrahge was high, probably because histology showed that all areas with intra-plaque hemorrhage were less than one week old. Therefore, we incorporated this T1w TFE pulse sequence in our MRI scan protocol. The other four MR pulse sequences (black-blood T1w TSE, T2w TSE, PDw TSE and partial T2w TSE) were chosen because they were previously used by others ${ }^{11,12,14,15,16,23}$ at start of this PhD study. We developed an algorithm for the identification of the four main plaque components (calcification, lipid core, intra-plaque hemorrhage and fibrous tissue) on the images of these five MR pulse sequences. The algorithm was developed for qualitative and semiquantitative plaque assessment. Again, the detection rate for intra-plaque hemorrhage was high, as well as for calcification, lipid core, and fibrous tissue for both qualitative and semi-quantitatively analysis. The sensitivity and specificity for the LRNC (lipid core and/or intra-plaque hemorrhage) was excellent $(93 \%$ and $96 \%$ and $76 \%$ and $100 \%$, for qualitative and semiquantitative rSI assessment, respectively). Because the accuracy of the semi-quantitative assessment was comparable to the qualitative assessment and the semi-quantitative assessment was quite timeconsuming, we limited ourselves to qualitative assessment for the next studies.

In another study we prospectively tested the algorithm on a larger series of patients. Additionally, we compared the multisequence results for LRNC quantification with the results solely based on single sequence T1w TFE images because Moody et $\mathrm{al}^{27}$ found high sensitivity and specificity for the identification of LRNC with hemorrhage using solely T1w magnetization prepared three-dimensional gradient echo MR images (comparable to our T1w TFE images). Our study found that multisequence MRI, using the algorithm, resulted in poor results for quantification of calcification and lipid core, moderate quantification of intra-plaque hemorrhage and good quantification of LRNC and fibrous tissue, while sensitivity and specificity for the presence of LRNC were still very good. ${ }^{32}$ Our standardized MR image assessment is important and probably more objective compared to methods used by others, ${ }^{33}$ although this needs to be studied in the future. The quantification of LRNC based on solely T1w TFE images showed similar results as for multisequence MRI. This is an important finding because quantification of LRNC based on single 
sequence T1w TFE MRI is much easier and faster than quantification of LRNC using multisequence MRI.

To interpret the better performance of multisequence MRI for the separate plaque components in the initial studies ${ }^{25,26}$ compared to the prospective study ${ }^{32}$ two main items are important: the methodology used and the way the algorithm works. Both initial studies focussed only on selected areas in the plaque, while the prospective study judged the whole plaque. The selected plaque areas were areas that could be easily delineated, while areas were delineation was difficult were neglected. The reason we did this in the initial studies ${ }^{25,26}$ was to aim at pure rSls for the various plaque components. Furthermore, the study in which the algorithm was developed ${ }^{26}$ used selected areas not blinded for histology, while the MR readers of the prospective study ${ }^{32}$ were blinded for histology. Moreover, in the study in which the algorithm was developed the performance of the algorithm was calculated on the same dataset, which provided the best possible results. A retrospective analysis on the data of the prospective study showed that the majority of areas histologically classified as lipid core were predicted by the algorithm as intra-plaque hemorrhage and it was noticed that lipid core was often hyperintense on T1w TFE images in contrast to the findings from the limited number of lipid cores ( $n=5$ and $n=6$, respectively) in the initial studies. ${ }^{25,26}$ In histology, it is hard to demarcate intra-plaque hemorrhage from lipid core because intra-plaque hemorrhage diffusely mixes with the lipid core. With that in mind it is understandable that MRI with a much lower in-plane resolution than microscopy cannot differentiate intra-plaque hemorrhage from lipid core. Others ${ }^{17,33}$ experienced similar problems with the differentiation of lipid core and intra-plaque hemorrhage. Although the separate identification of intra-plaque hemorrhage is of great interest, ${ }^{22}$ the current possibility to identify and quantify the LRNC (with or without intra-plaque hemorrhage) has great potential to study the LRNC over time in relation to symptoms and/or medical treatment. Murphy et $\mathrm{al}^{34}$, who used a similar MR pulse sequence (T1w magnetization prepared three-dimensional gradient echo MR images) as our T1W TFE sequence, showed that this sequence may be used to screen patients at risk of stroke for complicated plaques (LRNC with intra-plaque hemorrhage). They showed that the prevalence of high signal intensity in carotid plaque (suggestive for 
complicated plaque) was significantly greater in the carotid plaque ipsilateral to the symptoms than at contralateral side. The advantage of our MRI protocol compared to that of Murphy et $\mathrm{al}^{34}$ is that we obtain images at higher spatial resolution $(0.39 \times 0.39 \mathrm{~mm})$, which allows for quantification of smaller regions. A disadvantage of multisequence MRI compared to the single sequence approach of $\mathrm{us}^{32}$ and Murphy et $\mathrm{al}^{34}$ is that the acquisition and interpretation of more MR pulse sequences is more time consuming and also more difficult to interpret than only one single MR pulse sequence. However, others ${ }^{33}$ showed good results for the identification of various other plaque components with multisequence MRI. The group of Takaya et $\mathrm{al}^{35}$ used their approach of MR image assessment $^{33}$ to demonstrate that MRI features of carotid plaque are associated with the occurrence of subsequent cerebrovascular events. They $^{35}$ showed that patients with an increase of $10 \%$ in LRNC size were 1.6 times more likely to suffer a cerebrovascular event. A hazard ratio of 1.6 seems not much, but the ranges in LRNC sizes are large, which means that the percentages increase in LRNC size can be large as well and consequently significant increase the risk of stroke. Patients with a LRNC in the carotid artery (any size) were 4.4 times more likely to suffer a cerebrovascular event, however this was not statistical significant. ${ }^{35}$ Another interesting study was performed by Hatsukami et $\mathrm{al}^{36}$, who used a time of flight (TOF) MR pulse sequence and showed that this sequence can be used to assess fibrous cap thickness and cap rupture. The identification of a ruptured fibrous cap with MRI was highly associated with recent transient ischemic attack. ${ }^{37}$ It was shown that patients with a thin or ruptured fibrous cap were 10 and 23 times more likely to have suffered a recent transient ischemic attack (TIA) or stroke, respectively compared to patients with a thick fibrous cap. These publications about fibrous cap assessment with a TOF MR pulse sequence are very exciting, however still single center findings. In future studies we will include a TOF MR pulse sequence to our scan protocol.

Given the importance to obtain additional risk factors of stroke we liked to investigate whether our T1w TFE MR image assessment of the LRNC may be a suitable method to study the relation between the size of the LRNC in carotid plaque and stroke. The search for risk factors of stroke is extremely important because a large group of patients who undergo 
endarterectomy (80\% of symptomatic patients with high-grade carotid stenosis) will not have a major stroke on medical treatment alone. We tested the hypothesis that the average size of the LRNC as quantified with T1w TFE image assessment in carotid plaques of symptomatic patients with a high-grade stenosis was higher compared to asymptomatic patients with a high-grade stenosis. Three individual MRI readers quantified significantly larger lipid-rich necrotic core (LRNC) in carotid atherosclerotic plaque of symptomatic patients than in asymptomatic patients. The larger LRNC of symptomatic patients supported the idea that an atherosclerotic plaque with a large lipid core is more prone to rupture and thus has an increased risk of causing ischemic events. However, one should be cautious with interpretation of measurements of LRNC quantities in individuals, because there was considerable overlap between the individual patients of the symptomatic and asymptomatic group. Future studies need to test whether the LRNC quantity as assessed with our single sequence T1w TFE approach may be used as an additional risk factor of stroke. Furthermore, our method has the potential to study the LRNC size longitudinally in relation to medical therapy.

Multisequence MRI as well as single sequence T1w TFE MRI showed to have high potential for non-invasive atherosclerotic plaque imaging. Developments in MRI hardware such as higher magnetic field strengths and new coil technologies are ongoing and will boost signal to noise ratio and/or improve in-plane resolution. This will further improve the possibilities of MRI for plaque imaging. Furthermore, the development of new pulse sequences might improve the performance of MRI. Although MR image assessment as described in this thesis was standardized, plaque analysis will be more objective with automatic plaque characterization. Recent published initial results on this subject showed to be promising. ${ }^{38,39}$

\section{Gadolinium based contrast agents}

Commercially available gadolinium contrast agents may also add to the potential of MR plaque imaging. Gadolinium-enhanced MRI might help to discriminate fibrous cap from lipid core ${ }^{40,41}$ and, moreover, it enables quantification of neovascularization. ${ }^{42} \mathrm{~A}$ symptomatic atherosclerotic plaque is associated with increased neovascularization. ${ }^{31}$ Novel contrast agents, like MS-325 (strong MR enhancement after binding to albumin) are 
being tested after initial observations ${ }^{43}$ of vessel wall enhancement in diseased aortoiliac vessels. It was speculated that this agent enters the plaque by leaking through diseased plaque capillaries. Initially, in our studies we were more interested in the potential of multisequence MRI for plaque imaging without gadolinium based contrast agents, because it is less complicated and cheaper. However, the growing evidence of improved results with the use of gadolinium convinced us to investigate the additional value of gadolinium in future MR imaging studies on atherosclerotic plaque. For the quantification of LRNC we reduced our MRI protocol from five MR sequences to just one, which created opportunities to add other promising techniques to our protocols.

Ultrasmall superparamagnetic particles of iron oxide (USPIOs) MR contrast agents

Additionally to morphological identification and quantification of atherosclerotic plaque components it is also very important to assess plaque inflammation, because atherosclerosis is considered as an inflammatory disease. ${ }^{1}$ One of the features of high-risk atherosclerotic plaques is a preponderance of macrophages. Macrophages are very interesting as target for imaging, because they are involved in all stages of atherosclerotic plaque development. The imaging of macrophages might give opportunities for early detection of disease and by that opportunities for early medical intervention to prevent future thrombo-embolic complications. MR imaging of macrophages means imaging on cellular level, which is highly challenging using the current commercial state-ofthe-art MRI scanners. A group of MR contrast agents, which showed to have great potential for this purpose are the ultrasmall superparamagnetic particles of iron oxide (USPIOs). These USPIOs showed to have great potential for the imaging of macrophages in atherosclerotic plaque of rabbits. ${ }^{44,45}$ We were the very first who demonstrated USPIO uptake in macrophages within human carotid plaque and also showed in vivo signal changes on the MR images after USPIO administration. ${ }^{46}$ These findings were confirmed by others. ${ }^{47,48}$ Although the results of these initial studies are very promising there are some limitations, which need to be improved. The MR pulse sequence ( $T 2^{*} \mathrm{~W}$ gradient echo), which is most sensitive to the susceptibility artifacts of the iron particles in the macrophages, has a 
low signal to noise ratio (SNR). Furthermore the USPIO contrast agents is a so-called negative contrast agent, which means that areas with uptake appear black. Positive contrast is easier to depict by the human eye. However, both SNR as well as negative contrast issues are technically solvable. There are numerous possibilities to improve SNR, like novel MR pulse sequences, reducing the spatial resolution, improved coils and by using higher magnetic field strength. Recently, a new MR pulse sequence was introduced that can generate positive USPIO contrast. ${ }^{49}$

So far a limited number of human studies using USPIO contrast agent were performed and larger (multi-center) trials are warranted with both symptomatic as asymptomatic patients. The relation between imaging findings and ischemic events and/or drug use needs to be investigated. A relatively new field of research is molecular imaging of macrophages, which combines the detection of macrophages with the assessment of their functional state, such as their activation status.

\section{Molecular imaging of atherosclerotic plaque using MRI}

Besides the high potential of morphological plaque imaging with multisequence $\mathrm{MRI}$ and the promising results with macrophage imaging using USPIO-enhanced MRI, other exciting developments are ongoing in the development of targeted contrast agents. The basis of the development of targeted contrast agents is to couple a protein or molecule specific ligand to the contrast agent, which then together will bind to the target protein or molecule. An example is a fibrin-binding contrast agent for coronary thrombus imaging in swine. ${ }^{50}$ In the future, this may have impact for patients with acute coronary syndromes. Targets on the molecular level are specific molecules, which express selectively and/or with increased uptake in areas prone to atherosclerotic lesion formation. Molecular imaging has advantages above morphological imaging because molecular imaging covers all phases of atherogenesis, while morphological imaging identifies pathology only in later phases of disease. In this context, molecular imaging may give possibilities for treatment in early stages of atherosclerosis. Examples of target molecules are vascular cell adhesion molecule 1 (VCAM-1, also expressed on macrophages) and $\mathrm{P}$ - and E-selectin. ${ }^{51}$ Promising examples are novel iron nanoparticles with affinity for VCAM-1 as shown in a mouse model ${ }^{52}$ and 
iron nanoparticles with affinity for E-selectin in a Matrigel model ${ }^{53}$. At this moment a lot of research is going on to investigate the ability to incorporate drugs in targeted contrast agents. ${ }^{54,55}$ In various ways drugs can be linked or dissolved within for example carrier lipid coatings or trapped within the carriers itself. These developments open ways for imaging-based drug delivery to selective areas of disease and may concentrate drugs to the diseased areas, which may lead to lower systemic concentrations of the drug and thus less side effects. An example is a study of Lanza et al. ${ }^{56}$, who treated smooth muscle cells in culture with a tissue factor-targeted MRI nanoparticle contrast agent loaded with the drug paclitaxel. Smooth muscle proliferation in the vessel wall indicates progression of atherosclerosis. The investigated substance caused a substantial reduction in smooth muscle cell proliferation in vitro. This effect could be visualized on T1w spin echo images at 4.7T and quantified with ${ }^{19}$ Fluorine MR spectroscopy. Although these results are promising, the effects of these drug-targeted contrast agents should eventually be studied in relation to altering course of therapy and clinical events.

\section{Other non-invasive imaging techniques for atherosclerosis}

Techniques that may compete with MRI are CT and nuclear techniques as PET (positron emission tomography) and SPECT (single photon emission computer tomography). CT has a comparable in-plane resolution as MRI and CT is superior for the quantification of calcification. However, $\mathrm{CT}$ has difficulties to differentiate fibrous-rich and lipid-rich plaques. ${ }^{57} \mathrm{Up}$ to now, CT (without the combination with PET) seems not very promising for molecular imaging, regarding to the abcense of literature about this subject. Moreover, iodine based CT contrast agents still regularly give allergic side effects and rarely anaphylactoid shock. Another disadvantage compared to $\mathrm{MRI}$ is the use of ionizing radiation with $\mathrm{CT}$.

PET and SPECT are highly sensitive molecular imaging techniques, which are fast evolving. These techniques are capable to identify important cellular and molecular processes with radioactive isotopes (radionuclides). ${ }^{58}$ Ideally, the radionuclide binds specifically to the plaque constituent, is cleared relatively rapidly from the circulation to allow sufficient contrast between plaque and blood pool and uptake in adjacent structures should be minimal. Although the number of radionuclides are 
extensive, availability of the radionuclide is important for clinical application. At present Fluorine-18-deoxyglucose (FDG) is widely available and showed to have potential to detect plaque inflammation in humans. ${ }^{58,59,60}$ FDG-PET alone provides not enough anatomical resolution so that for the differentiation between atherosclerotic plaque and other adjacent metabolic active structures (for example inflammed lymph nodes) co-registration with other imaging modalities like $\mathrm{CT}^{58,59,60}$ or $M R \mathrm{I}^{61}$ is important. At present, PET-CT is widely available, while PET-MRI is more or less at the start of development.

Another promising substance (ligand) is Annexin-A5, ${ }^{58,62}$ a protein that binds to phospatidylserine (target), which is expressed on apoptotic cells. It is suggested that apoptosis (or programmed cell death) is associated with atherosclerotic plaque rupture. ${ }^{63}$ Nuclear imaging of phospatidylserine expression a rabbit model using labelled annexin A5 may identify vulnerable plaques at risk of rupture. ${ }^{64}$ Clinical studies of annexin-A5 were performed in myocardial infarction, cardiac allograft rejection, heart failure and carotid atherosclerosis. ${ }^{62}$ Kietselaer et $\mathrm{al}^{65}$ performed a study on four patients and showed in two patients with recent transient ischemic attack (TIA) that uptake of labeled annexin A5 as imaged with SPECT correlated with histological proven unstable carotid plaques. The other two patients of this study who had had transient ischemic attacks three months before imaging showed no annexin-A5 uptake and histological stable plaques.

\section{Outlook and conclusions}

The recognition that the risk of cardiovascular disease is largely determined by plaque composition rather than the degree of arterial stenosis $^{2,3}$ emphasized the lack of diagnostic imaging modalities for appropriate risk assessment of thrombo-embolic complications. In this thesis we demonstrated that MRI may play an important role for imaging of atherosclerosis. We showed the abilities of standardized multisequence as well as single sequence T1w TFE MR image assessment for the identification and quantification of the lipid-rich necrotic core (LRNC), and we showed the ability of USPIO-enhanced MRI for the detection of macrophage-rich regions within the atherosclerotic plaque of symptomatic patients with high-grade carotid stenosis. Future studies need to evaluate 
the value of our findings in asymptomatic patients at high risk for atherosclerotic trombo-embolic disease. Follow up studies, which study the high-risk components, such as the size of the LRNC, in relation to symptoms are warranted, as well as studies that evaluate progression and regression of plaques in relation to therapy. Furthermore, it needs to be studied whether standardized MR image assessment of carotid plaque can lead to a better patient selection of patients that will benefit of endarterectomy or carotid stenting. The MRI evaluation of coronary plaque is considerably more difficult than imaging the carotid plaque. The distance between the MRI coils and the coronary arteries is quite large and will decrease signal to noise ratio. Furthermore, the coronary plaques are of much smaller size and often tortuous, which makes MR imaging a challenge. Motion of the heart and diaphragma is another limiting factor for the appropriate MR imaging of the coronary plaque. However, MRI techniques are evolving very fast and provide the necessary tools to overcome these barriers. Botnar et $\mathrm{al}^{66}$ and Fayad et $\mathrm{al}^{67}$ were the first to demonstrate that non-invasive MRI was able to assess the coronary artery wall with submillimeter in-plane resolution. At present, progress may be achieved by higher magnetic field strengths, new coil technology, development of novel MR pulse sequences, use of intravenous contrast agents and improved post-processing techniques.

It is important to realize that the concept of the "vulnerable plaque" is not one of "one shoe fits all". ${ }^{68}$ The atherosclerotic plaque has many vulnerable aspects, such as the LRNC and macrophages as well as specific proteins and molecules. Furthermore, other extrinsic factors like blood thrombogenicity and the distribution of cerebral blood flow ${ }^{69}$ may influence the risk of thrombo-embolic complications. Therefore, it needs to be emphasized that MRI will not be a sole modality for plaque imaging and many other imaging techniques like PET and SPECT will be important. It is likely that multi-modality imaging will be important in the future. Moreover, imaging findings can also be correlated with for example serum markers of inflammation ${ }^{70}$ and microemboli detection ${ }^{71}$ as surrogate indicators of plaque vulnerability. 
It is clear that atherosclerotic plaque imaging needs a multidisciplinary approach. Atherosclerotic plaque imaging, with $\mathrm{MRI}$ as the most promising non-invasive and non-ionizing technique, provides opportunities to detect vulnerable plaques in vivo and prevent thromboembolic complications. 


\section{References}

1. Ross R. Atherosclerosis - An inflammatory disease. N Engl J Med. 1999;340:115-126.

2. Falk E, Shah PK, Fuster V. Coronary plaque disruption. Circulation. 1995;92:657-671.

3. Virmani R, Kolodgie FD, Burke AP, Farb A, Schwartz SM. Lessons from sudden coronary death: a comprehensive morphological classification scheme for atherosclerotic lesions. Arterioscler Thromb Vasc Biol. 2000;20:1262-1275.

4. Beneficial effect of carotid endarterectomy in symptomatic patients with high-grade carotid stenosis. North American Symptomatic Carotid Endarterectomy Trial Collaborators. N Engl J Med. 1991;325:445-453.

5. MRC European Carotid Surgery Trial: interim results for symptomatic patients with severe $(70-99 \%)$ or with mild $(0-29 \%)$ carotid stenosis. European Carotid Surgery Trialists' Collaborative Group. Lancet. 1991;337:1235-1243.

6. Randomised trial of endarterectomy for recently symptomatic carotid stenosis: final results of the MRC European Carotid Surgery Trial (ECST). Lancet. 1998;351:1379-1387.

7. Barnett HJ, Taylor DW, Eliasziw M, Fox AJ, Ferguson GG, Haynes RB, Rankin RN, Clagett GP, Hachinski VC, Sackett DL, Thorpe KE, Meldrum $\mathrm{HE}$, Spence JD. Benefit of carotid endarterectomy in patients with symptomatic moderate or severe stenosis. North American Symptomatic Carotid Endarterectomy Trial Collaborators. N Engl J Med. 1998;339:1415-1425.

8. Rothwell PM, Eliasziw M, Gutnikov SA, Fox AJ, Taylor DW, Mayberg MR, Warlow CP, Barnett HJ. Analysis of pooled data from the randomised controlled trials of endarterectomy for symptomatic carotid stenosis. Lancet. 2003;361:107-116.

9. Little WC, Constantinescu M, Applegate R, Kutcher MA, Burrows MT, Kahl FR, Santamore WP. Can coronary angiography predict the site of a subsequent myocardial infarction in patients with mild-to-moderate coronary artery disease? Circulation. 1988;78:1157-1166. 
10. Giroud D, Li JM, Urban P, Meier B, Rutishauer W. Relation of the site of acute myocardial infarction to the most severe coronary arterial stenosis at prior angiography. Am J Cardiol. 1992;69:729-732.

11. Shinnar M, Fallon JT, Wehrli $S$, Levin $M$, Dalmacy $D$, Fayad ZA, Badimon JJ, Harrington M, Harrington E, Fuster V. The diagnostic accuracy of ex vivo MRI for human atherosclerotic plaque characterization. Arterioscler Thromb Vasc Biol. 1999;19:2756-2761.

12. Serfaty JM, Chaabane L, Tabib A, Chevallier JM, Briguet A, Douek PC. Atherosclerotic plaques: classification and characterization with T2weighted high-spatial-resolution MR imaging-- an in vitro study. Radiology. 2001;219:403-410.

13. Coombs BD, Rapp JH, Ursell PC, Reilly LM, Saloner D. Structure of plaque at carotid bifurcation: high-resolution MRI with histological correlation. Stroke. 2001;32:2516-2521.

14. Toussaint JF, LaMuraglia GM, Southern JF, Fuster V, Kantor HL. Magnetic resonance images lipid, fibrous, calcified, hemorrhagic, and thrombotic components of human atherosclerosis in vivo. Circulation. 1996;94:932-938.

15. Fayad ZA, Fuster V. Clinical imaging of the high-risk or vulnerable atherosclerotic plaque. Circ Res. 2001;89:305-316.

16. von Ingersleben G, Schmiedl UP, Hatsukami TS, Nelson JA, Subramaniam DS, Ferguson MS, Yuan C. Characterization of atherosclerotic plaques at the carotid bifurcation: correlation of highresolution MR imaging with histologic analysis-- preliminary study. Radiographics. 1997; 17:1417-1423.

17. Yuan C, Mitsumori LM, Ferguson MS, Polissar NL, Echelard D, Ortiz G, Small R, Davies JW, Kerwin WS, Hatsukami TS. In vivo accuracy of multispectral magnetic resonance imaging for identifying lipid-rich necrotic cores and intraplaque hemorrhage in advanced human carotid plaques. Circulation. 2001;104:2051-2056.

18. Van Damme $H$, Vivario $M$, Boniver J, Limet $R$. Histologic characterization of carotid plaques. Cardiovasc Pathol. 1994;3:9-17.

19. Gutstein DE, Fuster, V. Pathophysiology and clinical significance of atherosclerotic plaque rupture. Cardiovasc Res. 1999;41:323-333. 
20. Naghavi M, Libby P, Falk E, Casscells SW, Litovsky S, Rumberger J, Badimon JJ, Stefanadis C, Moreno P, Pasterkamp G, Fayad Z, Stone PH, Waxman S, Raggi P, Madjid M, Zarrabi A, Burke A, Yuan C, Fitzgerald PJ, Siscovick DS, de Korte CL, Aikawa M, Juhani Airaksinen KE, Assmann G, Becker CR, Chesebro JH, Farb A, Galis ZS, Jackson C, Jang IK, Koenig W, Lodder RA, March K, Demirovic J, Navab M, Priori SG, Rekhter MD, Bahr R, Grundy SM, Mehran R, Colombo A, Boerwinkle E, Ballantyne C, Insull W, Jr., Schwartz RS, Vogel R, Serruys PW, Hansson GK, Faxon DP, Kaul S, Drexler H, Greenland P, Muller JE, Virmani R, Ridker PM, Zipes DP, Shah PK, Willerson JT. From vulnerable plaque to vulnerable patient: a call for new definitions and risk assessment strategies: Part I. Circulation. 2003;108:1664-1672.

21. Falk E. Stable versus unstable atherosclerosis: Clinical aspects. Am Heart J. 1999;138:S421-S425.

22. Kolodgie FD, Gold HK, Burke AP, Fowler DR, Kruth HS, Weber DK, Farb A, Guerrero LJ, Hayase M, Kutys R, Narula J, Finn AV, Virmani R. Intraplaque hemorrhage and progression of coronary atheroma. $N$ Engl J Med. 2003;349:2316-2325.

23. Yuan C, Mitsumori LM, Beach KW, Maravilla KR. Carotid atherosclerotic plaque: noninvasive MR characterization and identification of vulnerable lesions. Radiology. 2001;221:285-299.

24. Cai JM, Hatsukami TS, Ferguson MS, Small R, Polissar NL, Yuan C. Classification of human carotid atherosclerotic lesions with in vivo multicontrast magnetic resonance imaging. Circulation. 2002;106:13681373.

25. Cappendijk VC, Cleutjens KB, Heeneman S, Schurink GW, Welten R, Kessels AG, Van Suylen RJ, Daemen MJ, Van Engelshoven JM, Kooi $M E$. In vivo detection of hemorrhage in human atherosclerotic plaques with magnetic resonance imaging. JMRI. 2004;20:105-110.

26. Cappendijk VC, Cleutjens KB, Kessels AG, Heeneman S, Schurink GW, Welten RJ, Mess WH, Daemen MJ, van Engelshoven JM, Kooi ME. Assessment of human atherosclerotic carotid plaque components with multisequence MR imaging: initial experience. Radiology. 2005;234:487-492. 
27. Moody AR, Murphy RE, Morgan PS, Martel AL, Delay GS, Allder S, MacSweeney ST, Tennant WG, Gladman J, Lowe J, Hunt BJ. Characterization of complicated carotid plaque with magnetic resonance direct thrombus imaging in patients with cerebral ischemia. Circulation. 2003;107:3047-3052.

28. Chu B, Kampschulte A, Ferguson MS, Kerwin WS, Yarnykh VL, O'Brien KD, Polissar NL, Hatsukami TS, Yuan C. Hemorrhage in the atherosclerotic carotid plaque: a high-resolution MRI study. Stroke. 2004;35:1079-1084.

29. Kampschulte A, Ferguson MS, Kerwin WS, Polissar NL, Chu B, Saam T, Hatsukami TS, Yuan C. Differentiation of intraplaque versus juxtaluminal hemorrhage/thrombus in advanced human carotid atherosclerotic lesions by in vivo magnetic resonance imaging. Circulation. 2004;110:3239-3244.

30. Leu HJ. [Histological age determination of arterial and venous thrombi and emboli]. Vasa. 1973;2:265-274.

31. McCarthy MJ, Loftus IM, Thompson MM, Jones L, London NJ, Bell PR, Naylor AR, Brindle NP. Angiogenesis and the atherosclerotic carotid plaque: an association between symptomatology and plaque morphology. J Vasc Surg. 1999;30:261-268.

32. Cappendijk VC. In vivo quantification of the lipid-rich necrotic core in atherosclerotic plaque with standardized MR image assessment. Submitted. 2006.

33. Saam T, Ferguson MS, Yarnykh VL, Takaya N, Xu D, Polissar NL, Hatsukami TS, Yuan C. Quantitative evaluation of carotid plaque composition by in vivo MRI. Arterioscler Thromb Vasc Biol. 2005;25:234-239.

34. Murphy RE, Moody AR, Morgan PS, Martel AL, Delay GS, Allder S, MacSweeney ST, Tennant WG, Gladman J, Lowe J, Hunt BJ. Prevalence of complicated carotid atheroma as detected by magnetic resonance direct thrombus imaging in patients with suspected carotid artery stenosis and previous acute cerebral ischemia. Circulation. 2003;107:3053-3058. 
35. Takaya N, Yuan C, Chu B, Saam T, Underhill H, Cai J, Tran N, Polissar $\mathrm{NL}$, Isaac C, Ferguson MS, Garden GA, Cramer SC, Maravilla KR, Hashimoto B, Hatsukami TS. Association Between Carotid Plaque Characteristics and Subsequent Ischemic Cerebrovascular Events. A Prospective Assessment With MRI--Initial Results. Stroke. 2006;37:818823.

36. Hatsukami TS, Ross R, Polissar NL, Yuan C. Visualization of fibrous cap thickness and rupture in human atherosclerotic carotid plaque in vivo with high-resolution magnetic resonance imaging. Circulation. 2000;102:959-964.

37. Yuan C, Zhang SX, Polissar NL, Echelard D, Ortiz G, Davis JW, Ellington $E$, Ferguson MS, Hatsukami TS. Identification of fibrous cap rupture with magnetic resonance imaging is highly associated with recent transient ischemic attack or stroke. Circulation. 2002;105:181185.

38. Hofman JM, Branderhorst WJ, ten Eikelder HM, Cappendijk VC, Heeneman S, Kooi ME, Hilbers PA, ter Haar Romeny BM. Quantification of atherosclerotic plaque components using in vivo MRI and supervised classifiers. Magn Reson Med. 2006;55:790-799.

39. Liu F, Xu D, Ferguson MS, Chu B, Saam T, Takaya N, Hatsukami TS, Yuan C, Kerwin WS. Automated in vivo segmentation of carotid plaque MRI with Morphology-Enhanced probability maps. Magn Reson Med. 2006;55:659-668.

40. Wasserman BA, Smith WI, Trout HH, 3rd, Cannon RO, 3rd, Balaban RS, Arai AE. Carotid artery atherosclerosis: in vivo morphologic characterization with gadolinium-enhanced double-oblique MR imaging initial results. Radiology. 2002;223:566-573.

41. Takaya N, Cai J, Ferguson M, Yarnykh VL, Chu B, Saam T, Polissar N, Sherwood J, Cury R, Anders R, Broschat K, Hinton D, Furie K, Hatsukami T, Yuan C. Intra- and interreader reproducibility of magnetic resonance imaging for quantifying the lipid-rich necrotic core is improved with gadolinium contrast enhancement. JMRI. 2006;24:203210. 
42. Kerwin W, Hooker A, Spilker M, Vicini P, Ferguson M, Hatsukami T, Yuan C. Quantitative magnetic resonance imaging analysis of neovasculature volume in carotid atherosclerotic plaque. Circulation. 2003;107:851-856.

43. Maki JH, Wilson GJ, Lauffer RB, Weiskoff RM, Yuan C. Apparent vessel wall inflammation detected using MS-325, blood pool contrast agent Proc. Intl. Soc. Magn. Reson. Med.; 2001.

44. Ruehm SG, Corot C, Vogt P, Kolb S, Debatin JF. Magnetic resonance imaging of atherosclerotic plaque with ultrasmall superparamagnetic particles of iron oxide in hyperlipidemic rabbits. Circulation. 2001;103:415-422.

45. Schmitz SA, Coupland SE, Gust R, Winterhalter S, Wagner S, Kresse M, Semmler W, Wolf KJ. Superparamagnetic iron oxide-enhanced MRI of atherosclerotic plaques in Watanabe hereditable hyperlipidemic rabbits. Invest Radiol. 2000;35:460-471.

46. Kooi ME, Cappendijk VC, Cleutjens KB, Kessels AG, Kitslaar PJ, Borgers $M$, Frederik PM, Daemen MJ, van Engelshoven JM. Accumulation of ultrasmall superparamagnetic particles of iron oxide in human atherosclerotic plaques can be detected by in vivo magnetic resonance imaging. Circulation. 2003;107:2453-2458.

47. Trivedi RA, U-King-Im JM, Graves JG, Cross JJ, Horsley J, Goddard MJ, Skepper JN, Quartey G, Warburton E, Joubert I, Wang L, Kirkpatrick PJ, Brown J, Gillard JH. In vivo detection of macrophages in human carotid atheroma. Temporal dependence of Ultrasmall Superparamagnetic Particles of Iron Oxide-enhanced MRI. Stroke. 2004;35:1631-1635.

48. Trivedi RA, Mallawarachi C, JM UK-I, Graves MJ, Horsley J, Goddard MJ, Brown A, Wang L, Kirkpatrick PJ, Brown J, Gillard JH. Identifying inflamed carotid plaques using in vivo USPIO-enhanced MR imaging to label plaque macrophages. Arterioscler Thromb Vasc Biol. 2006;26:1601-1606.

49. Briley-Saebo KC, Mani V, Hyafil F, Fayad Z. Positive contrast MR imaging of in vivo atherosclerosis in a rabbit model using ultrasmall superparamagnetic iron oxide particles Proc. Intl. Soc. Mag. Reson. Med.; 2006. 
50. Botnar RM, Buecker A, Wiethoff AJ, Parsons EC, Jr., Katoh M, Katsimaglis G, Weisskoff RM, Lauffer RB, Graham PB, Gunther RW, Manning WJ, Spuentrup E. In vivo magnetic resonance imaging of coronary thrombosis using a fibrin-binding molecular magnetic resonance contrast agent. Circulation. 2004;110:1463-1466.

51. Libby P, Ridker PM, Maseri A. Inflammation and atherosclerosis. Circulation. 2002;105:1135-1143.

52. Kelly KA, Allport JR, Tsourkas A, Shinde-Patil VR, Josephson L, Weissleder R. Detection of vascular adhesion molecule-1 expression using a novel multimodal nanoparticle. Circ Res. 2005;96:327-336.

53. Kang HW, Torres D, Wald L, Weissleder R, Bogdanov AA. Targeted imaging of human endothelial-specific marker in a model of adoptive cell transfer. Lab Invest. 2006;86:599-609.

54. Wickline SA, Lanza GM. Nanotechnology for molecular imaging and targeted therapy. Circulation. 2003;107:1092-1095.

55. Wickline SA, Neubauer AM, Winter P, Caruthers S, Lanza G. Applications of nanotechnology to atherosclerosis, thrombosis, and vascular biology. Arterioscler Thromb Vasc Biol. 2006;26:435-441.

56. Lanza GM, Yu X, Winter PM, Abendschein DR, Karukstis KK, Scott MJ, Chinen LK, Fuhrhop RW, Scherrer DE, Wickline SA. Targeted antiproliferative drug delivery to vascular smooth muscle cells with a magnetic resonance imaging nanoparticle contrast agent: implications for rational therapy of restenosis. Circulation. 2002;106:2842-2847.

57. Cordeiro MA, Lima JA. Atherosclerotic plaque characterization by multidetector row computed tomography angiography. J Am Coll Cardiol. 2006;47:C40-47.

58. Davies JR, Rudd JH, Weissberg PL, Narula J. Radionuclide imaging for the detection of inflammation in vulnerable plaques. J Am Coll Cardiol. 2006;47:C57-68.

59. Ben-Haim S, Israel O. PET/CT for atherosclerotic plaque imaging. $O J$ Nucl Med Mol Imaging. 2006;50:53-60.

60. Okane K, Ibaraki M, Toyoshima H, Sugawara S, Takahashi K, Miura S, Shimosegawa E, Satomi J, Kitamura K, Satoh T. (18)F-FDG accumulation in atherosclerosis: use of $\mathrm{CT}$ and MR co-registration of thoracic and carotid arteries. Eur $\mathrm{J}$ Nucl Med Mol Imaging. 2006;33:589-594. 
61. Davies JR, Rudd JH, Fryer TD. Identification of culprit lesions after transient ischaemic attack by combined 18F-fluorodeoxyglucose positron emission tomography and high resolution magnetic resonance imaging. Stroke. 2006. In press.

62. Boersma HH, Kietselaer BL, Stolk LM, Bennaghmouch A, Hofstra L, Narula J, Heidendal GA, Reutelingsperger CP. Past, present, and future of annexin A5: from protein discovery to clinical applications. J Nucl Med. 2005;46:2035-2050.

63. Kolodgie FD, Narula J, Guillo P, Virmani R. Apoptosis in human atherosclerotic plaques. Apoptosis. 1999;4:5-10.

64. Kolodgie FD, Petrov A, Virmani R, Narula N, Verjans JW, Weber DK, Hartung D, Steinmetz N, Vanderheyden JL, Vannan MA, Gold HK, Reutelingsperger CP, Hofstra L, Narula J. Targeting of apoptotic macrophages and experimental atheroma with radiolabeled annexin $\mathrm{V}$ : a technique with potential for noninvasive imaging of vulnerable plaque. Circulation. 2003;108:3134-3139.

65. Kietselaer BL, Reutelingsperger $\mathrm{CP}$, Heidendal GA, Daemen MJ, Mess WH, Hofstra L, Narula J. Noninvasive detection of plaque instability with use of radiolabeled annexin A5 in patients with carotid-artery atherosclerosis. N Engl J Med. 2004;350:1472-1473.

66. Botnar RM, Stuber M, Kissinger KV, Kim WY, Spuentrup E, Manning WJ. Noninvasive coronary vessel wall and plaque imaging with magnetic resonance imaging. Circulation. 2000;102:2582-2587.

67. Fayad ZA, Fuster V, Fallon JT, Jayasundera T, Worthley SG, Helft G, Aguinaldo JG, Badimon JJ, Sharma SK. Noninvasive in vivo human coronary artery lumen and wall imaging using black-blood magnetic resonance imaging. Circulation. 2000;102:506-510.

68. Kereiakes DJ. The Emperor's clothes: in search of the vulnerable plaque. Circulation. 2003;107:2076-2077.

69. Hendrikse J, van Raamt AF, van der Graaf Y, Mali WP, van der Grond J. Distribution of cerebral blood flow in the circle of Willis. Radiology. 2005;235:184-189.

70. Weiss CR, Arai AE, Bui MN, Agyeman KO, Waclawiw MA, Balaban RS, Cannon RO, 3rd. Arterial wall MRI characteristics are associated with elevated serum markers of inflammation in humans. J Magn Reson Imaging. 2001;14:698-704. 
71. Hellings WE, Ackerstaff RG, Pasterkamp G, De Vries JP, Moll FL. The carotid atherosclerotic plaque and microembolisation during carotid stenting. J Cardiovasc Surg (Torino). 2006;47:115-126. 


\section{Summary}

MRI of atherosclerosis -

Identification and quantification of carotid plaque components 
Atherosclerosis is a systemic disease of the arterial vessel wall and is one of the leading causes of disabilities and death in industrialized societies. Atherosclerosis can develop because of intrinsic -genetic- causes (for example in familiar hypercholesterolemia), extrinsic causes (for example smoking and an excessive fatty diet) or combinations of both intrinsic and extrinsic causes. Atherosclerotic vessel wall changes and subsequent atherosclerotic plaque formation start early in life and progress during ageing. At present, the indication for therapeutic surgical or radiological interventions in atherosclerotic disease is determined by symptoms and the degree of stenosis.

In the last decade pathological studies have shown that certain plaque features are highly associated with cardiovascular syndromes. In advanced atherosclerosis the core of the plaque can contain lipid-rich substances, hemorrhage and inflammatory cells (macrophages), together called the lipid rich necrotic core. The luminal site of a healthy artery is covered by a single layer of endothelial cells, but in advanced plaques the vulnerable core is covered by a fibrous cap. Rupture of this fibrous cap causes contact of the blood with the thrombogenic plaque contents and consequently gives rise to thrombo-emboli and/or luminal occlusion. Therefore, rupture of the fibrous cap is the predominant underlying process in the pathogenesis of acute cardiovascular syndromes (i.e. stroke and myocardial infarction). Currently daily clinical practice lacks noninvasive diagnostic tests for atherosclerotic plaque assessment and luminography is still the most prominent imaging tool for cardiovascular disease. For patients with suspected carotid plaque disease estimation of the degree of stenosis by luminography or duplex ultrasonography is important to determine the treatment strategy but plaque assessment may improve the identification of patients who could benefit from surgery.

A particularly attractive modality to gain insight into plaque morphology is magnetic resonance imaging (MRI). MRI has high potential for atherosclerotic plaque imaging, because of its high soft tissue contrast, high in-plane resolution, and excellent reproducibility and repeatability. In the present thesis we asked ourselves the question whether vulnerable atherosclerotic plaque components could be identified and quantified in the carotid artery with standardized in vivo MRI. To be able to perform 
these studies over one-hundred patients with carotid stenosis larger than $70 \%$ were recruited.

\section{Assessment of intra-plaque hemorrhage}

In chapter 2, we showed that it is feasible to identify intra-plaque hemorrhage in carotid plaque with in vivo MRI. Previous pathological studies indicated that intra-plaque hemorrhage is a high-risk plaque component and that the presence of hemorrhage in the lipid-rich necrotic core (LRNC) may be a stimulus for growth of the LRNC. Therefore, it is important to be able to identify intra-plaque hemorrhage in vivo. Althoug initially several authors experienced difficulties to image this component with spin echo pulse sequences, Moody et al were able to detect intraplaque hemorrhage in the carotid artery of patients suffering anterior cerebral circulation ischemia using a $\mathrm{T} 1$ weighted $(\mathrm{T} 1 \mathrm{w})$ turbo field echo (TFE) sequence (also referred to as a T1w magnetization-prepared $3 \mathrm{D}$ gradient echo sequence). Nevertheless, the in-plane resolution in that study was limited and no slice-by-slice comparison between MRI and histology was performed. The purpose of our first study was therefore to test the performance of a T1W TFE MR pulse sequence to detect intraplaque hemorrhage in vivo with high-resolution $\mathrm{MRI}(0,39 \times 0,49 \mathrm{~mm}$ in-plane resolution). Higher spatial-resolution images have the advantage that smaller areas of interest can be detected. In this study more than $80 \%$ of the histological proven areas of intra-plaque hemorrhage could be detected on T1w TFE images with a high inter-observer agreement. Since plaques containing hemorrhage are considered to carry a higher rupture risk, this technique could be important in the selection of patients at risk for stroke. Future studies need to investigate the signal characteristics of intra-plaque hemorrhage at T1w TFE imaging over time and the relation with the onset of symptoms.

\section{Development of standardized MR image assessment}

In chapter 3, a systematic multisequence MRI approach was developed for the identification of various atherosclerotic plaque components. Moreover, we investigated the feasibility of a quantitative approach besides the more commonly used qualitative approach. Although several other groups previously demonstrated the usefulness of ex vivo and in 
vivo multisequence $M R I$ for the identification plaque components, it was still unclear which pulse sequence or combination of pulse sequences was the best for identification of the various components. An algorithm was developed using a logistic regression model for the identification of these components (calcification, lipid core, intra-plaque hemorrhage, fibrous tissue and lipid-rich necrotic core (LRNC), were LRNC is defined as the sum of lipid core and intra-plaque hemorrhage). The optimal combination of five MR pulse sequences and cutoff values for the plaque components were determined. The LRNC, which is regarded as a key indicator of plaque vulnerability, could be identified with high sensitivities and specificities of $93 \%$ and $96 \%$ and $76 \%$ and $100 \%$, for qualitative and quantitative rSI assessment, respectively. Because qualitative assessment performed similar to quantitative assessment while the latter method was considerably more time-consuming, qualitative assessment of plaque components with this algorithm is recommended.

\section{Accuracy of MRI to quantify the lipid-rich necrotic core}

In chapter 4, the accuracy of quantification of the lipid-rich necrotic core (LRNC) using the multisequence algorithm developed in chaper 3, was determined. Additionally, the accuracy of quantification of LRNC using just a single T1w TFE pulse sequence was determined since others found high sensitivity and specificity (both $84 \%$ ) for the identification of LRNC with hemorrhage using this sequence. Present thinking is that a larger LRNC confers a higher risk for thrombo-embolic complications. However, the amount of LRNC associated with an increased risk for thrombo-embolic events is unknown. Previous histological have demonstrated that carotid plaques can contain a wide range of quantities of LRNC and therefore the mere presence of high-risk components will probably not be sufficient to assess plaque vulnerability. Therefore, accurate quantification the exact amount of LRNC is an important prerequisite before MRI plaque imaging can be implemented as a clinical tool. Standardized MR image assessment using the algorithm described in chapter 3, as well as solely T1w TFE images by two individual readers blinded for histology showed high inter -and intra-reader agreement and good correlation between histology and MRI for the LRNC size. Sensitivities and specificities were at least $70 \%$ for a large range of 
histological cutoff points. Since these T1w TFE images were obtained in less than 4 minutes as opposed to a total scan time of about 25 minutes for a multisequence approach, quantification of LRNC based solely on T1w TFE images will be much easier to implement in clinical practice. Not only image acquisition but also analysis of the images is considerably faster and more straightforward compared to multisequence imaging.

An important secondary finding from this study is that the algorithm could not differentiate lipid core from intra-plaque hemorrhage. Retrospectively analysis of a subset of data showed that large lipid cores without hemorrhage often showed high signal intensities on T1w TFE images, resulting in incorrect classification as intra-plaque hemorrhage by the algorithm. A reason for this finding is that we, as well as other groups, experienced difficulties to demarcate hemorrhage in the LRNC in histology, because intra-plaque hemorrhage is often distributed diffusely in the LRNC. This might also explain why we could accurately quantify LRNC with MRI, while the accuracy of quantification of intra-plaque hemorrhage was only moderate and quantification of lipid core was poor.

Studies specifically aiming to quantify the LRNC could restrict the MR protocol to acquisition of T1w TFE images, which is accurate, reliable and fast for this purpose.

Comparison of the LRNC in symptomatic and asymptomatic carotid patients

In chapter 5, we investigated whether the average amount of LRNC in carotid plaques as quantified on MR images of symptomatic patients with high-grade carotid stenosis is higher compared to asymptomatic patients with high-grade carotid stenosis. Although large randomized clinical trials have found a reduced risk of stroke in patients with high-grade atherosclerotic carotid artery narrowing who underwent endarterectomy, the vast majority $(80 \%)$ of these patients will not have a major stroke on medical treatment alone within three years. The present study was an initial attempt to investigate whether quantification of the LRNC on T1w TFE MR images may be a suitable method to study the relationship between the size of the LRNC in carotid plaque and stroke. Therefore T1w TFE imaging needs to be able to differentiate symptomatic from asymptomatic patients. Three MR readers, unaware of any additional 
medical information, individually assessed all carotid plaques and found very consistent results, reflected by high intra- and interreader correlations. All observers found significantly larger lipid-rich necrotic core (LRNC) in carotid atherosclerotic plaque of symptomatic as opposed to asymptomatic patients. The larger LRNC in symptomatic patients supports the idea that an atherosclerotic plaque with a large lipid core is more prone to rupture and thus carries an increased risk of causing ischemic events. Future studies are needed to prospectively investigate whether the LRNC size as assessed with our approach may be used as an additional parameter of the risk of stroke. Furthermore, our method has potential to study the LRNC size longitudinally in relation to stroke and medical therapy.

\section{Plaque inflammation}

In chapter 6, in a proof of concept study, we investigated whether a novel type of MR contrast agent of the class of ultrasmall superparamagnetic particles of iron oxide (USPIO) is taken up in human carotid plaques in vivo and whether the MR signal changes in plaques after USPIO administration. Besides the presence of a large lipid-rich necrotic core, one of the features of high-risk atherosclerotic plaques is a preponderance of macrophages. MR imaging of macrophages means imaging on cellular level, which is highly challenging using the current commercial state-of-the-art MRI scanners because the relatively low signal to noise ratio that can be achieved. Previous to our study, there were no data available on the uptake of USPIO in human carotid plaque in vivo. Experimental studies in hyperlipidemic rabbits had shown that ultrasmall superparamagnetic particles of iron oxide (USPIOs) accumulate in plaques with a high macrophage content and that this induced MR signal changes. However, the dose USPIO administered in the rabbits was about twenty times higher than allowed for our human study.

Not only did we prove that USPIOs are taken up and can be visualized in human carotid atherosclerotic plaques, but we also found that USPIOs were mainly taken up in macrophages in rupture prone and ruptured lesions. The accumulation of USPIO caused a significant signal decrease in regions of interest in the vessel wall of T2* weighted MR images acquired after 24 hours. There was also signal decrease after 72 hours but this was 
not statistically significant. Our results were later confirmed by Trivedi et al, who found an optimal time window for post-contrast imaging between 24 and 36 hours. The main problem of the use of the USPIO contrast agent was that the effects were best visualized with $\mathrm{T} 2 *$ weighted images, which have a inherently low signal to noise ratio (SNR). However, there are numerous possibilities to improve SNR, like novel MR pulse sequences that allow for positive instead of negative contrast, reducing the spatial resolution, improved radiofrequency coils and by using scanners with a higher main magnetic field strength.

\section{Discussion}

In chapter 7, the findings of the current thesis are discussed in relation to the published literature about atherosclerotic plaque imaging with MRI as well as other promising imaging modalities. The discussion concludes with a future outlook.

\section{Conclusions}

In the studies performed in the present thesis we found that MR imaging based solely on T1w TFE images can accurately, reproducibly and fast, quantify the vulnerable carotid plaque component lipid-rich necrotic core.

Furthermore, we concluded that MRI may have the potential to assess the relation of LRNC size and the risk of stroke because it was found that T1w TFE MR imaging found a significant larger average amount of lipidrich necrotic core in symptomatic patients compared to asymptomatic patients with high-grade carotid stenosis. However, the overlap in average amounts of lipid-rich necrotic core of symptomatic and asymptomatic patients needs cautious interpretation of these amounts in individuals.

Our multisequence MR algorithm consisting of five MR pulse sequences (T1w TFE, PDw TSE, T2w TSE, T1w TSE and partial T2w TSE) could accurately quantify fibrous tissue but could not quantify calcification. Moreover, our algorithm was not able to differentiate lipid core from intra-plaque hemorrhage. The quantification of lipid-rich 
necrotic core with multisequence $\mathrm{MRI}$ was accurate and as good as quantification based on solely T1w TFE images.

Another important feature of vulnerable plaques is the preponderance of macrophages (plaque inflammation). In a proof of concept study we demonstrated that in vivo MR imaging of macrophages in predominantly ruptured and rupture-prone carotid atherosclerotic plaque is feasible. Uptake of USPIOs by macrophages resulted in a focal signal decreases in the MR images.

The results of the studies presented in this thesis are important for clinical practice, because MRI offers a non-invasive tool to identify and quantify LRNC in atherosclerotic plaques. Future studies are needed to prospectively investigate high-risk features of carotid plaque like LRNC and plaque inflammation in relation to future stroke, as well as progression and regression of these plaque features in relation to therapy. Furthermore, it needs to be studied whether standardized MR image assessment of carotid plaque can lead to better selection of patients who will benefit of endarterectomy or carotid stenting. 


\section{Nederlandse samenvatting}

“Magnetic resonance imaging" van aderverkalking -

Identificatie en kwantificatie van plaque componenten in de halsslagader 
Aderverkalking, ook wel atherosclerose genoemd, is een aandoening van de vaatwanden van slagaders die gepaard gaat met een hoge morbiditeit en mortaliteit. Aderverkalking kan ontstaan door intrinsieke -genetische- oorzaken (bijvoorbeeld door familiaire hypercholesterolemie), extrinsieke oorzaken (bijvoorbeeld door roken en/of een overdadig vet dieet) of door combinaties van beide. In feite is de naam aderverkalking onjuist en onvolledig. Onjuist omdat het niet de aders maar de slagaders betreft en onvolledig omdat de veranderingen in de vaatwand niet alleen gebaseerd zijn op kalkvorming. De vaatwand verandering begint al op jeugdige leeftijd en neemt toe met de jaren. In eerste instantie treedt er een vettige verdikking op van de vaatwanden. Deze vaatwand verdikking kan ontwikkelen tot gevorderde atherosclerotische plaque, welke meestal eerst excentrisch groeit zonder vaatvernauwing te veroorzaken. Dit is een belangrijk gegeven, omdat uit eerdere publicaties over dit onderwerp is gebleken dat in het overgrote deel van de gevallen de cardiovasculaire syndromen, zoals men kent het herseninfarct en hart infarct, ontstaan in slagaders met geen of milde vaatvernauwing. Daarnaast zijn er in de laatste vijftien jaar steeds meer pathologische studies verschenen die aantoonden dat bepaalde plaque kenmerken in grote mate geassocieerd zijn met cardiovasculaire syndromen.

De wand van een gezonde slagader bestaat uit drie lagen, de binnenste laag is de intima en bestaat uit slechts één laag cellen (het "endotheel"), de middelste laag wordt gevormd door spiercellen (de "media") en de buitenste laag bestaat uit vetrijk steunweefsel (de "adventitia"). Bij atherosclerose is de intima aangedaan. In vergevorderde atherosclerotische plaque is de intima veranderd en bestaat uit vetrijke substanties, bloed en ontstekingscellen ("macrofagen"), samen het atheroom genoemd ("lipidrich necrotic core"). Het atheroom is bedekt door een dikkere structuur, de fibreuze kap, bestaande uit onder andere fibroblasten, myofibroblasten en lagen collageen, en is uiteindelijk weer bedekt door een laag endotheel. Door scheuren in deze fibreuze kap komt de atheromateuze kern in contact met bloed waardoor stolsels ontstaan. Delen van deze stolsels kunnen losraken (embolus) en stroomafwaarts vast komen te zitten. Indien er onvoldoende bloed via alternatieve routes komt (collateralen) dan sterft het weefsel achter de verstopte slagader af. Dit fenomeen noemt men infarct. Het scheuren van de fibreuze kap wordt beschouwd als een van de 
meest cruciale stappen in het ontstaan van acute cardiovasculaire syndromen.

Op dit moment wordt de indicatie voor operatief of interventie radiologisch ingrijpen bij vaatpatiënten met een plaque in de halsslagader bepaald door symptomatologie van de patiënt en de mate van vaatvernauwing. De beeldvormende technieken zoals nu gebruikt in de klinische praktijk zijn gericht op het vaststellen van de mate van vernauwing van de slagader. Er zijn momenteel geen goede reproduceerbare non-invasieve testen om atherosclerotische plaque componenten te identificeren en kwantificeren. Het afbeelden van plaque kan mogelijk leiden tot een betere selectie van patiënten die baat hebben bij therapie.

"Magnetic resonance imaging" (MRI) is bij uitstek een interessante beeldvormende modaliteit om kennis over de plaque morfologie te verwerven. MRI bezit de eigenschappen die nodig zijn voor adequate afbeelding van een atherosclerotische plaque, zoals een hoge contrast resolutie, een hoge spatiële resolutie, en tevens goede reproduceerbaarheid. In dit proefschrift is onderzocht of bij mensen met behulp van MRI belangrijke plaque kenmerken kunnen worden geïdentificeerd en gekwantificeerd. Hiervoor zijn meer dan honderd patiënten met een vernauwing van de halsslagader van meer dan $70 \%$ onderzocht.

\section{Identificatie van bloed in de plaque}

In hoofdstuk 2 is een onderzoek beschreven over de mogelijkheid om met MRI bloedingen in een plaque af te beelden. Een plaque bloeding moet immers beschouwd worden als risicovol, omdat bloed in de plaque de atheromateuze kern ("lipid-rich necrotic core") kan stimuleren tot groei. Uit pathologische studies blijkt dat een patiënt met een grote atheromateuze kern meer kans heeft op een hersen- of hartinfarct. Voor aanvang van onze studies werd in alle publicaties over dit onderwerp beschreven dat MRI de plaque bloeding niet goed kon identificeren. In deze publicaties werd gebruik gemaakt van zogenaamde spin echo technieken. Eén publicatie van Moody en collega's beschreef dat dit wel lukte met een T1 gewogen (T1w) turbo field echo (TFE) MRI techniek bij patiënten met (dreigende) infarcten in de voorste hersencirculatie. De 
spatiële resolutie in de studie van Moody was beperkt en bovendien werd geen één op één vergelijking gemaakt van de MRI beelden met de gouden standaard, de histologie. De TFE MRI techniek is een gradiënt echo techniek, waarbij een beter contrast ontstaat tussen de plaque bloeding en de directe omgeving. In onze studie werd de spatiële resolutie verhoogd naar $0,39 \times 0,49 \mathrm{~mm}$, waarmee kleinere gebiedjes met bloed gedetecteerd kunnen worden. De belangrijkste resultaten van de studie waren dat de bevindingen van twee beoordeelaars van de MRI beelden goed overeen kwamen en dat deze beoordeelaars meer dan $80 \%$ van de histologisch bewezen plaque bloedingen detecteerden. Deze T1w TFE techniek heeft daarmee potentie om risicovolle atherosclerotische plaque te detecteren.

Ontwikkeling van gestandaardiseerde MRI beoordeling van plaque componenten

In hoofdstuk 3 wordt een onderzoek beschreven over de identificatie van diverse plaque componenten door middel van een systematische analyse van verschillende MR beelden. Ons MRI protocol bestond uit vijf verschillende MR wegingen, zogenaamde $M R$ puls sequenties, gezamenlijk een "multisequence MR plaque imaging protocol genoemd. Elke MR weging geeft beelden met specifieke contrast eigenschappen en dit kan in belangrijke mate helpen om de diverse plaque componenten te differentiëren. Een nadeel is dat hierdoor een overvloed aan data ontstaat (vijf beelden van elk niveau van de plaque), wat snel tot verwarring leidt. Hoewel verschillende andere onderzoeksgroepen de waarde van ex vivo en in vivo "multisequence" MRI hebben beschreven voor de identificatie van plaque componenten, bieden deze publicaties geen duidelijkheid over de beste combinaties van MR wegingen voor specifieke plaque componenten. Daarom hebben wij zelf een algoritme ontwikkeld voor de identificatie van de verschillende plaque componenten (kalk, lipide kern, plaque bloeding, fibreus weefsel en het atheroom ("lipid-rich necrotic core"), gedefinieerd als de som van lipide kern en bloeding) met behulp van een logistisch regressie model. Het algoritme differentieert kalk, lipide kern, plaque bloeding en fibreus weefsel in drie vaste stappen, waarbij elke stap staat voor de optimale MR weging combinatie voor een specifieke component. Tevens werden voor alle van MR wegingen de bijbehorende afkappunten bepaald (signaal intensiteit). De analyse werd 
bovendien zowel kwantitatief als kwalitatief uitgevoerd, waarbij gefocust is op het atheroom ("lipid-rich necrotic core"). Het atheroom wordt beschouwd als een indicator van de risicovolle plaque en wordt met ons algoritme geïdentificeerd met hoge sensitiviteit en specificiteit $193 \%$ en $96 \%$, en $76 \%$ en $100 \%$, voor de kwalitatieve en kwantitatieve analyse, respectievelijk). De kwalitatieve analyse was zeker zo goed als de kwantitatieve analyse, terwijl de kwantitatieve analyse veel tijdrovender was. Daarom wordt de kwalitatieve analyse aanbevolen voor nieuwe studies.

Kwantificatie van de grootte van het plaque atheroom ("lipid-rich necrotic core")

In hoofdstuk 4 wordt een studie beschreven waarin onderzocht is hoe nauwkeurig MRI de grootte van het plaque atheroom kan kwantificeren. In deze studie wordt gebruik gemaakt van het multisequentie algoritme uit hoofdstuk 3. Omdat andere onderzoekers reeds een hoge sensitiviteit en specificiteit (beide $84 \%$ ) hadden aangetoond voor de identificatie van atheroom met alleen T1w TFE beelden, wordt in deze studie ook onderzocht hoe nauwkeurig atheroom grootte kan worden gekwantificeerd met alleen T1w TFE.

De huidige opinie is dat een groter atheroom een hoger risico geeft op trombo-embolische complicaties. Wat de precieze hoeveelheid atheroom is, dat geassocieerd is met een verhoogd risico is echter onbekend. Histologische data beschrijven zeer verschillende hoeveelheden atheroom in plaque in de halsslagader. Hieruit blijkt dat identificatie van het atheroom alleen onvoldoende zal zijn om atherosclerotische plaque te categoriseren. MRI moet dus zowel kleine als grote hoeveelheden atheroom nauwkeurig kunnen kwantificeren om in de toekomst bruikbaar te kunnen zijn in de klinische praktijk.

In deze studie kwantificeerden twee onafhankelijke MR beoordeelaars de atheroom grootte in plaque in de halsslagader, geblindeerd voor de gouden standaard, de histologie. Zij waren het in hun beoordeling in hoge mate met elkaar eens ("interreader agreement"), als ook met zichzelf bij een tweede onafhankelijke beoordeling een aantal maanden later ("intra-reader agreement"). Dit gold zowel voor de multisequentie beoordeling als ook voor de T1w TFE beoordeling. Er was een hoge 
correlatie tussen MRI en histologie voor atheroom grootte. De sensitiviteit en specificiteit voor atheroom grootte waren op zijn minst $70 \%$ voor een uiteenlopende reeks van histologische afkappunten. Ook dit gold voor zowel multisequentie MRI als voor beoordeling op basis van alleen T1w TFE.

Een belangrijk gegeven is dat de T1w TFE beelden veel sneller gemaakt zijn (in minder dan 4 minuten), dan de vijf MR wegingen van het multisequence protocol (25 minuten). Verder is niet alleen de scantijd voor T1w TFE korter, maar ook de beoordeel tijd aanzienlijk korter en eenvoudiger. Kwantificatie van atheroom grootte met T1w TFE beelden zal dus makkelijker in de klinische praktijk te implementeren zijn.

Deze studie toonde ook dat het multisequentie algoritme niet kan differentiëren tussen de lipide kern en plaque bloeding. Retrospectieve analyse op een deel van de data toonde dat de lipide kern zonder bloed vaak een hoge MR signaal intensiteit heeft, in tegenstelling van wat in hoofdstuk 3 gevonden was (lage signaal intensiteit van de lipide kern). Dit resulteerde in een verkeerde classificatie door het algoritme van de lipide kern in plaque bloeding. Ook andere wetenschappers hebben beschreven dat het lastig kan zijn om de plaque bloeding af te grenzen binnen het atheroom, omdat de bloeding zo diffuus in het atheroom verdeeld is. Dit is een tweede verklaring waarom ons MRI multisequentie algoritme de grootte van het atheroom wel nauwkeurig kan kwantificeren, maar dat de kwantificatie van de grootte van plaque bloeding slechts redelijk is en dat de grootte van de lipide kern slecht bepaald kan worden.

Tot slot, toekomstige studies die alleen geïnteresseerd zijn in de kwantificatie van atheroom grootte kunnen hun MR protocol beperken tot alleen T1w TFE beelden, omdat dit een techniek is die hiervoor zowel nauwkeurig, betrouwbaar als snel is.

\section{Vergelijking van symptomatische en asymptomatische patiënten met MRI}

In hoofdstuk 5 is een studie beschreven waarin werd onderzocht of de atheroom ("lipid-rich necrotic core") grootte gemiddeld hoger is in plaque van symptomatische patiënten dan in plaque van asymptomatische patiënten. Het gaat voor beide groepen om patiënten met een hooggradige vernauwing in de halsslagader. 
Uit grote gerandomiseerde klinische onderzoeken is gebleken dat patiënten met een hooggradige vernauwing in de halsslagader én symptomen hiervan, een verminderd risico op een herseninfarct hebben als ze zich laten opereren aan de vernauwing ("endarterectomie"). Echter, het is ook uitgezocht dat een groot aantal van deze patiënten ( $80 \%$ van de symptomatische patiënten met een hooggradige vernauwing) de eerste drie jaar sowieso geen groot herseninfarct krijgt met alleen conservatieve therapie.

Deze studie was een initiële studie om uit te zoeken of de kwantificatie van atheroom grootte met T1w TFE beelden geschikt is om de relatie te bestuderen tussen atheroom grootte en het herseninfarct. Hiervoor moet T1w TFE verschil tussen de groepen kunnen aantonen. Drie MR beoordelaars, geblindeerd voor alle klinische informatie, hebben onafhankelijk een grote hoeveelheid plaque van symptomatische en asymptomatische patiënten beoordeeld en vonden zeer consistente resultaten (hoge intra- en interreader correlaties). Zij scoorden significant grotere atheroom grootte in plaques van symptomatische patiënten vergeleken met asymptomatische patiënten. Het gemiddeld grotere atheroom in symptomatische patiënten ondersteunt de opinie dat een atherosclerotische plaque met een groot atheroom meer kans heeft op scheuring van de fibreuze kap en daarmee een hogere kans geeft op herseninfarcten. Het blijft wel belangrijk dat men bij individuen de metingen van atheroom grootte met voorzichtigheid interpreteert, omdat er een aanzienlijke overlap is in atheroom grootte tussen de individuele patiënten uit de symptomatische en asymptomatische patiënten groep.

Vervolg studies zijn gerechtvaardigd en moeten onderzoeken of atheroom grootte zoals wij dat kwantificeren daadwerkelijk geschikt is als extra parameter voor de inschatting van de kans op het herseninfarct. Ook heeft onze methode potentie om atheroom grootte te onderzoeken in relatie tot de tijd en in relatie tot therapie met behulp van seriële MRI.

\section{Ontsteking in de plaque}

In hoofdstuk 6 is een studie beschreven waarin onderzocht is of ultra kleine superparamagnetische deeltjes van ijzeroxide ("ultrasmall superparamagnetic particles of iron oxide", USPIO) opgenomen worden in 
plaque in de halsslagader van mensen. Ook is onderzocht of de MR signaal intensiteit veranderd na toediening van USPIO.

Het is erg belangrijk om ontsteking in de plaque te bestuderen, omdat atherosclerose wordt beschouwd als een ontstekingsziekte. Eén van de kenmerken van een risicovolle plaque is de overvloed van macrofagen (macrofagen zijn de opruimcellen van het afweersysteem). MRI van macrofaag-rijke plaque betekent beeldvorming op cellulair niveau en is, met de op dit moment beschikbare klinische MRI scanners, een enorme uitdaging.

Publicaties van dier-experimentele studies met konijnen met zeer vetrijke diëten hadden reeds aangetoond dat USPIO ophopen in plaque met veel macrofagen en dat hierdoor veranderingen in MR signaal worden geïnduceerd. Echter, de dosis USPIO die aan de konijnen werd gegeven was ongeveer twintig maal hoger als toegestaan bij mensen.

In onze studie werd aangetoond dat USPIO hoofdzakelijk opgenomen werden in macrofagen van zogenaamde geruptureerde plaque (dat wil zeggen: ruptuur van de fibreuze kap) of bijna geruptureerde plaque. De opname van USPIO zorgde voor een significante MR signaal daling in de vaatwand op zogenaamde T2* gewogen MR beelden, gemaakt 24 uur na intraveneuze toediening van de USPIO. Na 72 uur werd een statistisch niet significante MR signaal daling waargenomen. Onze studie is wereldwijd de eerste studie die resultaten publiceerde over de opname van USPIO in plaque in de halsslagader van mensen.

Onze resultaten werden later bevestigd door de groep van Gillard, die beschreven dat de optimale tijd voor beeldvorming ligt tussen de 24 en 36 uur na toediening van USPIO.

Het grootste probleem met het gebruik van USPIO is dat de effecten het best tot zijn recht komen op $\mathrm{T}^{*}$ gewogen MR beelden, terwijl deze beelden slechts een matige signaal ruis verhouding hebben. Er zijn meerdere mogelijkheden de signaal ruis verhouding te verbeteren in toekomstige studies, zoals: het gebruik maken van andere MR puls sequenties, het verminderen van de spatiële resolutie, verbeterde zend en ontvangst spoelen, en door gebruik te maken van sterkere magneetvelden. Het is recent ook mogelijk geworden om positief contrast te genereren in plaats van negatief contrast (dat wil zeggen dat er dan hoge signaal intensiteit is in gebieden van de vaatwand waar veel USPIO opname is 
geweest). Naar verwachting zijn beelden met positief contrast ten gevolge van USPIO opname in de vaatwand eenvoudiger te beoordelen.

\section{Discussie}

In hoofdstuk 7 worden de bevindingen van dit proefschrift besproken in samenhang met de internationale literatuur. Tevens worden alternatieve veelbelovende beeldvormende technieken besproken. Tot slot wordt een toekomst perspectief geschetst.

\section{Conclusies}

Uit de verschillende studies beschreven in dit proefschrift wordt geconcludeerd dat MR beeldvorming met alleen T1w TFE beelden een accurate, reproduceerbare en snelle manier biedt om het risicovolle atheroom ("lipid-rich necrotic core") te kwantificeren in plaque gelegen in de halsslagader.

Tevens wordt geconcludeerd dat MRI potentie heeft de relatie te bestuderen tussen de grootte van het atheroom en de kans op het herseninfarct, omdat met T1w TFE beelden gemiddeld een significant groter atheroom gevonden werd in plaque van symptomatische patiënten vergeleken met plaque van asymptomatische patiënten. Echter, uit onze resultaten blijkt ook dat er een aanzienlijke overlap is atheroom grootte tussen de symptomatische en asymptomatische patiënten en dit vraagt daarom om een voorzichtige interpretatie bij individuele patiënten.

Ons multisequence MR algoritme bevatte vijf MR pulse sequences (T1w TFE, PDw TSE, T2w TSE, T1w TSE and partial T2w TSE) waarmee een nauwkeurige kwantificatie mogelijk was voor de hoeveelheid fibreus weefsel, echter niet voor de hoeveelheid kalk. Ons algoritme was niet in staat de lipide kern te differentiëren van plaque bloeding. De kwantificatie van de atheroom grootte was met multisequence MRI even goed als met T1w TFE alleen.

Een overvloed aan macrofagen in een plaque (plaque ontsteking) is een ander kenmerk van een risicovolle plaque. Uit onze studie met USPIO blijkt dat macrofagen hoofdzakelijk ophopen in geruptureerde of bijna 
geruptureerde plaque in de halsslagader. Wij toonden aan dat USPIO opgenomen worden in deze macrofagen en dat dit leidde tot focaal signaal verlies op de $2^{*}$ MR beelden van de plaque.

De resultaten van de studies in dit proefschrift kunnen belangrijk zijn voor de klinische praktijk, omdat MRI een niet invasieve techniek is die de risicovolle kenmerken van atherosclerotische plaque kan identificeren en kwantificeren. Toekomstige studies worden aanbevolen om de relatie tussen deze risicovolle kenmerken van plaque in de halsslagader, zoals het atheroom en plaque ontsteking, en het optreden van een herseninfarct te onderzoeken. Ook seriële MRI studies naar de effecten van therapie op plaque progressie en regressie zijn nodig.

Uiteindelijk moet onderzocht worden of onze MRI technieken leiden tot een betere patiënten selectie, dat wil zeggen: een nauwkeurigere inschatting of een patiënt baat heeft bij operatie of stenting van de atherosclerotische halsslagader. 


\section{Dankwoord}


Dit proefschrift was niet tot stand gekomen zonder de hulp van veel anderen. Ik ben trots op het resultaat zoals het er nu ligt, en zeker ook dát het er ligt. Graag wil ik een ieder bedanken die een bijdrage heeft geleverd aan dit proefschrift. Ook wil ik de mensen bedanken, die me juist even niet aan dit proefschrift lieten denken.

Professor van Engelshoven, beste Jos, weinig opleiders zullen naast dat ze onderzoek belangrijk vinden, ook daadwerkelijk zo enorm in arts-onderzoekers investeren. Ook ik kreeg die kans en heb zo mijn horizon kunnen verbreden. Ik ben je hiervoor dankbaar.

Dr. Kooi, beste Eline, al heeft je nauwgezetheid me wel eens tot wanhoop gedreven, heeft het veel kwaliteit gebracht. Ook was het zonder jouw kennis van MRI niet gelukt. Ontzettend bedankt voor alles. Ik hoop dat je heel veel succes hebt met de diverse vervolgstudies.

Professor Daemen, beste Mat, je leerde mij de histologische coupes lezen en dit was een essentiële stap voor dit proefschrift. Dank ook voor je heldere en snelle commentaren op mijn manuscripten.

Dr. Heeneman, beste Sylvia, je hebt me in een bepaalde fase van het onderzoek enorm uit de brand geholpen. Daarbij had je een sterke eigenschap: je maakte de pathologie begrijpelijk voor mij en je kon je ook nog verplaatsen in de (mijn) radiologische denkwereld.

Dr. Cleutjens, beste Kitty, je bent heel belangrijk geweest in de USPIO studie, onmisbaar. Bedankt hiervoor!

Fons, statistisch magiër Kessels, hoofdstuk 3 draag ik aan jou op. Dat je mij de logistische regressie analyse hebt kunnen bijbrengen is een prestatie van formaat, maar ook het belang van een goede database. Dankjewel, ik heb veel van je geleerd. Én, je bent een prettig persoon.

Prof. Dr. Mess, beste Werner, de studie opzet was in beginsel te groot, waardoor onze ultrasonische samenwerking toch uitdoofde. Ik heb genoten van je bevlogenheid. Ook dank aan de medewerkers van de afdeling klinische neurofysiologie.

Alle leden van de beoordelingscommissie; Professor Limburg (voorzitter), Professor Kitslaar, Professor Verbrugge, Dr. Hofstra en Dr. van der Lugt. Een neuroloog, een vaatchirurg, een microbiologe, een cardioloog en een radioloog. Wat kan ik me meer wensen. 
De patiënten, die belangeloos meededen, terwijl er een operatie wachtte. Belangrijke informatie is verkregen. We zijn een stapje verder!

De vaatchirurgen die mij de patiënten hebben aangemeld, en in het bijzonder Geert Willem Schurink en Rob Welten (Heerlen). Fantastisch dat jullie ondanks jullie drukke werkzaamheden toch steeds aan me gedacht hebben. Tevens dank hiervoor aan Dr. Jan Tordoir, Professor Jacobs, Professor Kitslaar en Dr. Joep Teijink (Heerlen). Dank ook aan Dr. Verduin (Heerlen).

Mark Willemsen, leuk dat je jouw wetenschap stage bij mij hebt gedaan. Zonder kennis van de atherosclerotische plaque kom je er natuurlijk niet, dat had jij wel door. Dank voor je nuttige bijdrage.

Dr. R.J. van Suylen, beste Robert Jan, met overtuiging gaf je adviezen en de doorslaggevende stem in de histologische beoordelingen. Leuk om met je te werken.

Gé Hofland, radioloog te Venlo, je was direct bereid me te helpen om ook Venlo bij de studies te betrekken. Dat dit uiteindelijk niet van de grond gekomen is doet niets af aan deze zeer positieve houding. Ik heb het gewaardeerd.

Hetzelfde geldt voor Luc van den Akker, vaatchirurg te Sittard. Ik herinner me je inhoudelijke interesse. Dankjewel. Helaas had ik in Sittard concurrentie van een andere studie.

Diverse medewerkers van de afdeling pathologie voor het vervaardigen van vele histologische coupes.

Mijn collega's arts-assistenten en radiologen: nooit een onvertogen woord als ik vanwege het onderzoek afwezig was, maar juist interesse hoe het ging. Super!

In het bijzonder dank ik mijn meelevende kamergenoten (als zij weggingen en ik aan mijn tweede werkdag begon): Tim, Michiel, Boudewijn, Andreas en Jeroen. Ik hoop dat we elkaar nog vaak zien.

Mijn kamergenoot van het eerste uur, Vera. We zaten in de catacomben van het ziekenhuis, kijkend tegen een blinde muur. Jij hebt meer gevoel voor wetenschap en diplomatiek dan ik. lk hoop dat ik er iets van meegekregen heb. Bedankt voor je luisterend oor.

Tim Leiner, jij nogmaals: je bent een hardcore onderzoeker zonder kapsones maar met humor. Je hebt me gemotiveerd, uitstekend geholpen 
én was niet te beroerd samen met mij (en Boudewijn) een Tripel Karmeliet te drinken. Bedankt!

De MRI laboranten van het eerste uur (Henk, Etienne, Thea, Roland, Liesbeth, Evelien, Sonja, Axel, Dorke, Shahla en Kim), in het bijzonder Etienne Lemaire en Henk Schoenmakers. Jullie hebben meer verstand van MRI dan wij radiologen. Met jullie hulp leerde ik zelf al mijn patiënten scannen.

Romhild Hoogeveen (Philips Medical Systems), bedankt voor de optimalisatie van enkele MRI sequenties en de diverse uitnodigen voor leuke meetings.

Geertjan (AVD, azM), bedankt voor je hulp met de diverse figuren, het zijn tenslotte de beelden die het verhaal vertellen.

Marc Geerlings. Bedankt voor alle slimme computer hulp.

De dames van het secretariaat radiologie (Elfie, Astrid, Brigitte, Monique en Christianne): bedankt voor de prima hulp. Elly (secretaresse van Mat Daemen), bedankt voor de altijd vlotte bemiddeling.

Ine Kengen, beste Ine, in een handomdraai had je de lay-out voor elkaar. Hartstikke bedankt hiervoor.

Diverse opleiders uit mijn klinische verleden; Dr. K.G. Tan (chirurg, Albert Schweitzer ziekenhuis, Dordrecht), Prof. Dr. F.W.J. Hazebroek (kinderchirurg, SKZ, Rotterdam) en Prof. Dr. H.A. Bruining (chirurgintensivist, Erasmus MC, Rotterdam). Dit is een mooie plek om ook jullie te bedanken voor de perfecte klinische leerschool die jullie mij boden. Onbetaalbaar!

Eindelijk, vrienden en vriendinnen. Zonder de vrolijke tijden met jullie had ik het niet gekund. Rob, bijna Dr. Nijenhuis, zonder jouw komst in de azM dungeons had ik het behoorlijk saai gevonden. Eindelijk leven in de brouwerij...dankjewel. Erwin, amigo, je wist vaak beter dan ik wat mijn sterke en zwakke punten waren. Met enige inspanning kon je me de boel de boel laten...Ga zo door! Marc en Marieke, zonder "party van Dooren" geen party. Frank, goed dat je in Maastricht hebt gewoond, een positieve levensinstelling is goud waard. Ed en Edwin, lekker door de modder crossen en dan naar Sjiek. De andere Vlissingse boys, zeeuwen zijn niet stug, zeeland is cool! Ook dank aan allerlei anderen, met steeds blijvende interesse en gezellige en waardevolle bezoeken; Boudewijn (van Etten), James, Tony, Jan, Kim, Koert. 
Internationale Ronde Tafel 179 Maastricht. Bij het verschijnen van dit boekje ben ik zelf inmiddels geen Tafelaar meer, maar ik wil jullie toch zeker roemen. In twee jaar tijd werd een kleine 40.000 euro gedoneerd aan een kinderoncologisch onderzoek project van het azM. Zeer belangrijk, klasse!

$\mathrm{Pa}$, Fred, met het vorderen van dit proefschrift heb je al mijn karakter trekken voorbij zien komen. Ze staan niet in dit proefschrift, maar je kende ze al wel. Fijn dat je mijn vader bent.

$\mathrm{Ma}$, Hilde, weinigen zullen zoveel voor anderen doen dan jij. Erg bijzonder. Blij dat jij mijn moeder bent.

Angelique, zus, je wilde bedankt worden. Dan wel op mijn feestje komen.

Mijn paranimfen! Marc van ljken, beste Marc, een prettige gedachte dat een chirurg mij steunt. Je hebt altijd wel een mooie kijk op dingen. Michiel Cappendijk, beste $b(r)$ oer, op meerdere plaatsen in de wereld heb je naast me gestaan, meestal als lobbyist, en dat is altijd erg goed bevallen. We gaan maar weer werken vanuit het aloude STAR principe (Situation, Target, Action, Results). Heren, fijn dat jullie mij bijstaan.

Lieve Laura, elke dag met jou is een feest. Dankjewel. Ik kijk er naar uit de promotiedag met je te delen. Het lijkt me een bijzonder goed idee om samen op relaxed safari te gaan in Afrika. 


\section{Curriculum Vitae}




\section{Personal particulars}

$\begin{array}{lll}\text { Full name } & : & \text { Vincentius Carel Cappendijk } \\ \text { First name } & : & \text { Vincent } \\ \text { E-mail } & : & \text { vince2hawaii } @ \text { hotmail.com } \\ \text { Date of birth } & : & \text { 14 August } 1970 \\ \text { Place of birth } & : & \text { Eindhoven }\end{array}$

\section{Education}

June 1997

MD, Erasmus University Rotterdam

May 1995

August 1990

September 1989

May 1989

MSc in medicine, Erasmus University Rotterdam

Propedeuse medicine, Erasmus University Rotterdam

Start study medicine, Erasmus University Rotterdam

Finished pre-university education (V.W.O.), R.S.G. Scheldemond Vlissingen / Eckartcollege Eindhoven,

(subjects: Dutch language, English language, Physics, Biology, Mathematics A, Mathematics B, Geography, Chemistry)

\section{Work}

1May01-present : Combined resident radiology and $\mathrm{PhD}$ program, Maastricht University Hospital, Maastricht (Thesis: "MRI of atherosclerosis Identification and quantification of carotid plaque components")

$\begin{array}{lll}\text { 1Jan01-31Feb01 : } & \begin{array}{l}\text { Resident surgery (liver transplantation/ } \\ \text { esophagus surgery), Erasmus MC Rotterdam }\end{array} \\ \text { 1Jan00-31Dec00 : } \quad \begin{array}{l}\text { Resident surgical intensive care, Erasmus MC } \\ \text { Rotterdam }\end{array}\end{array}$


1Jan99-31Dec99 : Resident pediatric surgery, Sophia Children Hospital, Rotterdam

14Jul97-31Dec98 : $\quad$ Resident surgery, Albert Schweitzer ziekenhuis, Dordrecht

\section{Leisure}

Hockey, hiking, travelling, golf, ski and scuba diving. 


\section{List of publications}




\section{Publication (impact factor, number of citations on 16 January 2007)}

1. Hofman JM, Branderhorst WJ, ten Eikelder HM, Cappendijk VC, Heeneman S, Kooi ME, Hilbers PA, ter Haar Romeny BM. Quantification of atherosclerotic plaque components using in vivo $\mathrm{MRI}$ and supervised classifiers. Magn Reson Med; 2006 Apr;55(4):790-9. (3.5, not applicable)

2. Leiner T, Gerretsen S, Botnar R, Lutgens E, Cappendijk V, Kooi E, van Engelshoven J. Magnetic resonance imaging of atherosclerosis. Eur Radiol; 2005 Jun;15(6):1087-99. (2.4, 4x)

3. Cappendijk VC, Cleutjens KB, Kessels AG, Heeneman S, Schurink GW, Welten RJ, Mess WH, Daemen MJ, van Engelshoven JM, Kooi ME. Assessment of human atherosclerotic carotid plaque components with multisequence MR imaging: initial experience. Radiology; 2005;234:487-492. (5.4, 8x)

4. Cappendijk VC. Non-invasive new diagnostic techniques. Atherosclerotic plaque imaging with MRI. Rivista Italiana di Neurobiologica; 2004;suppl 1-2:33-36. (Not applicable)

5. Cappendijk VC, Cleutjens KB, Heeneman S, Schurink GW, Welten R, Kessels AG, Van Suylen RJ, Daemen MJ, Van Engelshoven JM, Kooi $\mathrm{ME}$. In vivo detection of hemorrhage in human atherosclerotic plaques with magnetic resonance imaging. JMRI. 2004;20:105-110. (2.5, 9x)

6. Kooi ME, Cappendijk VC, Cleutjens KB, Kessels AG, Kitslaar PJ, Borgers M, Frederik PM, Daemen MJ, van Engelshoven JM. Accumulation of ultrasmall superparamagnetic particles of iron oxide in human atherosclerotic plaques can be detected by in vivo magnetic resonance imaging. Circulation. 2003;107:2453-2458. (11.6, 90x) 
7. Cappendijk VC, van de Ven CP, Madern GC, Haverlag R, van Vugt AB, Hazebroek FWJ. Een opmerkelijke fractuurgenezing bij een 3 jarig kind. Ned Tijdschr Traumatologie 2000; 3: 89-90. (not applicable)

8. Cappendijk VC, van de Ven CP, Madern GC, Haverlag R, van Vugt AB, Hazebroek FWJ. Strength of youth: conservative treatment of segmental bone loss in children. J Trauma 2000; 49: 1123-5. $(1.7,1 \mathrm{x})$

9. Cappendijk VC, Hazebroek FWJ. The impact of diagnostic delay on the course of acute appendicitis. Arch Dis Child 2000; 83: 64-66.

Commentary on this paper in The Lancet 2000; 356: 787. $(1.8,14 \mathrm{x})$

10. Cappendijk VC, Mouthaan PJ. A true aneurysm of the tibioperoneal trunk. Case report and literature review. Eur J Vasc Endovasc Surg 1999; 18: 536-537. $(2.0,1 x)$

11. Cappendijk VC, Schütte $P R$, Tan KG. Cryo-stripping van de vena saphena magna. Scripta Phlebologica 1999; 7: 23-25. (not applicable)

12. Barker DP, Willetts B, Cappendijk VC, Rutter N. Capillary blood sampling: should the heel be warmed? Arch Dis Child Fetal Ed 1996; 74: $139-40 .(1.8,4 x)$

\section{Research awards}

- Pelerin prize 2005 (Award for best abstract and presentation Universtity Hospital Maastricht residents symposium; "Kwantificeren van atherosclerotische carotis plaque componenten met MRI")

- Ernst-Schering prize 2004 (award for the best abstract and presentation Dutch Radiological Society)

- Ernst-Schering prize 2003 (nomination for the best abstract and presentation Dutch Radiological Society) 